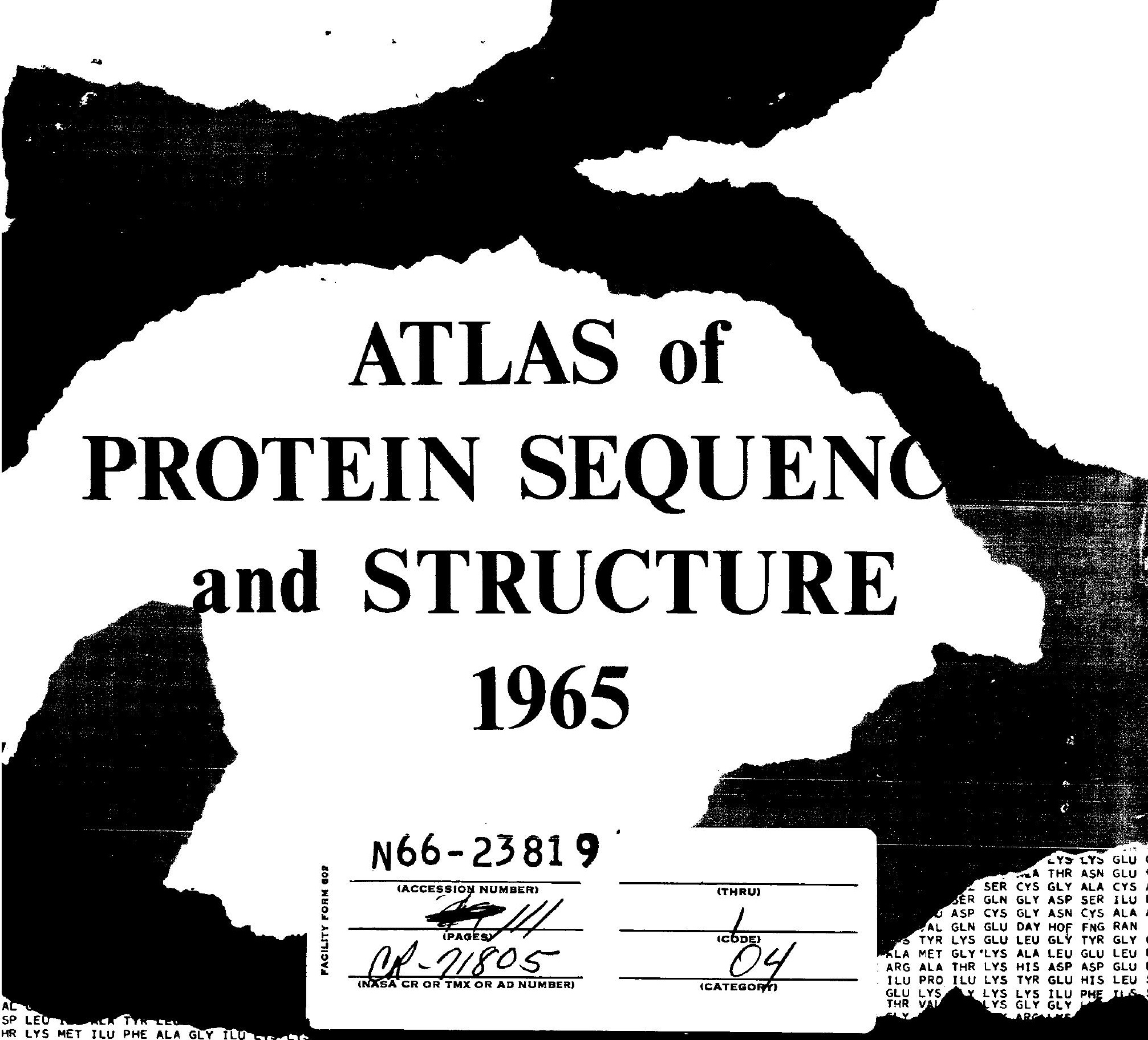

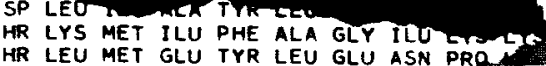

GPO PRICE $\$$

CFSTI PRICE(S) $\$$

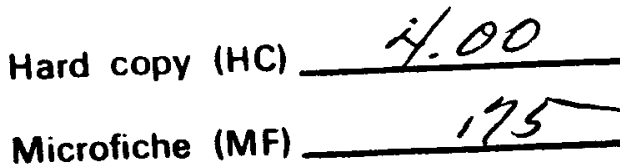

ff 653 July 65

National Biomedical Research Foundation 8600 16TH STREET Silver Spring, Maryland
Margaret O. Dayhoff Richard V Eck rie A. Chang R. Sochard 


\section{ATLAS of}

PROTEIN SEQUENCE and STRUC

\section{Margaret O. Dayhoff Richard V. Eck Marie A. Chang Minnie R. Sochard}

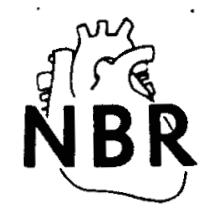

National Biomedical Research Foundation 8600 16TH STREET

Silver Spring, Maryland 
ATLAS OF PROTEIN SEQUENCE AND STRUCTURE 1965

Library of Congress Card Catalogue Number 65-29342

(C) Copyright 1965

by

The National Biomedical Research Foundation

Extra copies may be purchased from:

The National Biomedical Research Foundation

8600 16th Street, Silver Spring, Maryland 


\section{DEDICATION}

To all the investigators who have developed the techniques necessary for the grand accomplishments represented by this tabulation, and to all those who have spent so much tedious effort in their application.

We would gratefully appreciate receiving suggestions, corrections, new data (even if fragmentary or provisional), and references to any data omitted from this volume.

M. O. D.

R. V. E.

M. A. C.

M. R. S. 


\section{PREFACE}

This Atlas voluminously illustrates the triumph of experimental technique over the secretiveness of nature. Perhaps nowhere has the power of the scientific method been more brilliantly demonstrated than in the development of procedures for the study of the chemistry of life. As recently as twenty years ago, it was customary for biologists to have a hopeless attitude about biochemistry. Some details might be elicited, perhaps, but living things were thought to be so very complex and intricate that there surely was no hope of fully "understanding" them in all their chemical detail. Who, if he really comprehended the difficulty of the problem, would dare to think of man's ever knowing the detailed structure of a protein, for example, much less be able to synthesize it? Who would ever understand the mechanism of an enzyme as clearly as a chemist understands the details of an inorganic reaction? How could we ever hope to know the atomic details of a protein crystal?

Today some of these ambitions have already been attained, and the others no longer seem out of reach. We now rationally hope to be able to discover and understand the finest chemical details of living processes. These accomplishments and hopes have been made possible by the combined effect of several new approaches.

Techniques which permit the separation of chemically similar compounds have been developed for microgram samples. Among these are ion-exchange columns, paper chromatography, electrophoresis, and counter-current distribution. Radioactive tracer techniques and other micro-quantitative analytical procedures, often dependent on electronics and automation, have aided the analyses. X-ray crystallography, starting with the art of protein crystal production and ending with the processing of great numbers of experimental observations in the high-speed computer, has permitted a glimpse of three-dimensional structure.

Confidence in our understanding of experimental procedures and relationships among proteins has grown so great that sequences of amino acids are inferred from those found in homologous proteins. This technique requires only a small proportion of the analytical work needed to sequence a protein with no known relatives. The effectiveness of laboratory effort is thus magnified.

Some of the insights which have been developed cannot be attributed to any particular worker or school. Perhaps the greatest of these insights is that nature always uses "building blocks." A living cell is extremely complex and almost unimaginably intricate in detail. But it consists of a limited, understandable number of types of processes, reduplicated with variations. To understand the cell, we must have a few examples of each type of process, from which we can see the overall principles. For understanding, we need not work out the details of all the variations on these principles, although we may eventually choose to do so for medical or other practical reasons. Similarly, the analysis of such large, complex chemical molecules as proteins has been made possible by the recognition of their essential modularity, their buildingblock nature. Proteins are precise chemical structures built from regular subunits, 
not statistical mixtures or hopelessly intricate molecular conglomerates as was once thought. It is by means of the discovery and utilization of such building block principles, combined with the large-scale application of new and improved techniques, that we now dare hope to make all of living nature accessible to our understanding.

Hidden in the amino acid sequence of a protein is the chemical information that produces its three-dimensional structure. In the case of an enzyme, this structure forms locks into which the proper keys-cell chemicals-fit. By these locks, the enzymes bring the proper reactants together quickly, efficiently, and selectively. Uncatalyzed reactions cannot complete with such specifically catalyzed reactions; therefore, the presence of enzymes determines which reactions can take place in living chemistry. In many cases, if not all, this three-dimensional structure is fully determined by the information in the one-dimensional sequence. The folding is the thermodynamically most stable result of all the possible intermolecular forces, such as hydrogen bonds and hydrophobic bonds, which can form between the various links of the chain. In principle, if we knew these forces in detail, and if we had appropriate computer routines, we should then be able to determine the three-dimensional structure of a protein, given only its amino acid sequence.

Also hidden in the sequences is information about the genes which directed their synthesis. For each amino acid there are a small number of possible corresponding nucleotide triplets in the gene. That is, each protein sequence corresponds to a limited number of possible nucleotide sequences. When nucleotide mutations occur, the substitution of alternative amino acids is not random. Analysis of amino acid sequence data, considered as a mathematical puzzle, can help elucidate both the mathematical details of the genetic code and the structural aspects of the genetic mechanism.

Hidden in each family of homologous sequences is the story of its evolution. Simple organisms, caught in their primitive ecological niches, still preserve even today enzymes performing primeval functions, held relatively fixed by natural selection. Even the older proteins of man are preserved as living "fossils" in his metabolism.

Enmeshed also in homologous sequences are the records of the many thousands of mutational steps by which we can quantify a phylogenetic tree. Each amino acid link is a trait by which we can trace species evolution. By comparison, the traditional taxonomic criteria are extremely vague and uncertain. In the case of distant relationships, they often break down completely. A truly quantitative and inclusive system of phylogenetic classification would be of great help to comparative physiologists and other students of evolution.

Conspicuous in comparative human protein sequences is information of great medical-diagnostic value. A long series of abnormalities has been found to be attributable to single amino acid replacements. One such tragically serious disease is sickle-cell anemia.

To facilitate the theoretical study of the protein sequences which have already been so ingeniously and laboriously determined, we have undertaken this compilation. 
The information is kept in a compact, uniform format on punched cards. New information and corrections are easily inserted, while the text is kept accurate.

It is our intention to include the currently accepted amino acid sequence of every protein for which complete or substantial data is available. Uusally, only the definitive report giving the complete sequence from each laboratory will be referenced. If a substantial amount of work has been done on the same protein in other laboratories, their reports will also be referenced. We have also included some smaller peptides that have come to our attention. Unusual polypeptides which are presumably not produced by the genetic code have been omitted.

The format in which the Atlas is kept on punched cards is suitable for direct use in our computer programs. We use a three-letter code, which is a slight modification of the conventional notation, and also a mnemonic one-letter code which is clearer and much more suitable for certain comparative studies. We use a system of punctuation to describe the degree of confidence in each bond. Brief remarks are also included about the nature and function of the protein, and additional structural information such as the attachment of prosthetic groups, the location of S-S bonds, amino acids involved in active sites, and three-dimensional structures. In later editions we intend to include a section in which the alignment of all sequences of each family is given. Possibly we will also have sections on alleles and on mathematical methods and computer programs to treat the information.

This first edition is incomplete and imperfect and is intended mainly for distribution to investigators who have published protein sequence analyses, to acquaint them with the existence of this Atlas. We would gratefully appreciate their cooperation in making corrections, additions and suggestions for future editions. Since sequences are being reported in great numbers, we plan to bring out supplementary material in six months and a second edition in a year.

We thank all those who have assisted with this compilation, particularly Mr. Javier Albarran for his help with the computer aspects and Miss Lorrie Goldstein for her design of the cover.

The tabulations and computations were made at the University of Maryland Computer Science Center, College Park.

This work was supported by Grants GM-08710 and GM-12168 from the National Institutes of Health to the National Biomedical Research Foundation. 
TABLE OF CONTENTS

PAGE

PREFACE• • $\bullet \bullet \bullet \bullet \bullet \bullet \bullet \bullet \bullet \bullet \bullet \bullet \bullet \bullet \bullet \bullet \bullet \bullet \bullet \bullet \bullet \bullet$

0.001

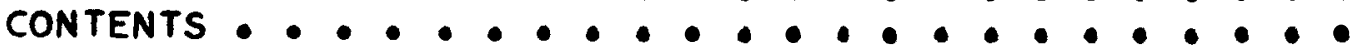

0.002

EXPLANATION OF NOTATION $\bullet \cdot \bullet \cdot \bullet \cdot \bullet \cdot \bullet \cdot \bullet \cdot \bullet$

0.010

I. CYTOCHROME C $\quad 1.000$

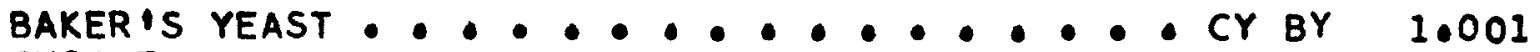

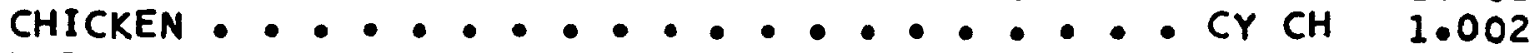

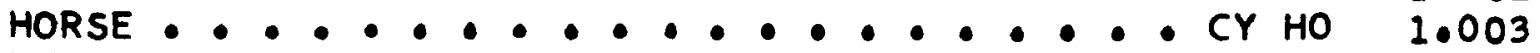

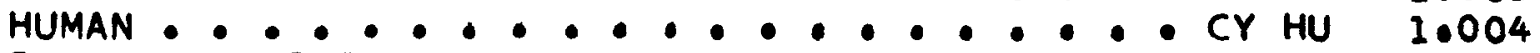

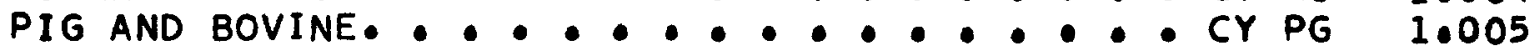

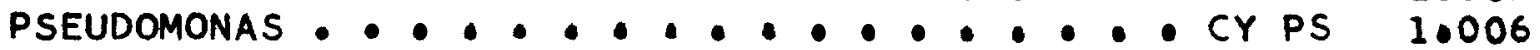

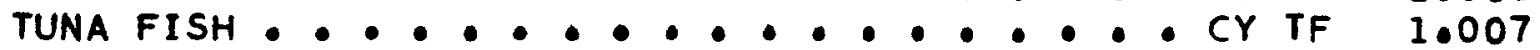

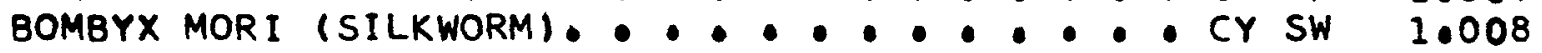

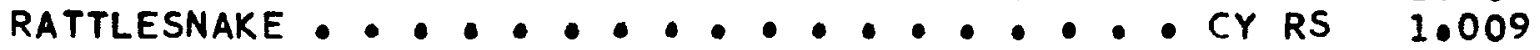

RHODOSPIRILLUM RUBRUM ............. CY RR 1.010

SALMON $\bullet \cdot \bullet \cdot \bullet \cdot \bullet \cdot \bullet \cdot \bullet \cdot \bullet \cdot \bullet \cdot \bullet$ CY SM 1.011

$\begin{array}{lr}\text { II. GLOBINS } & 2.000\end{array}$

HEMOGLOBINS 2.000

hUMAN alpha ................ Gl huHa 2.001

human beta ............. Gl huHB 2.002

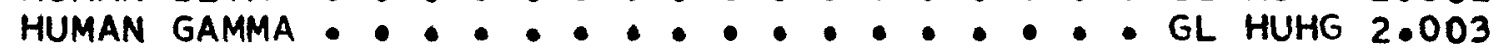

Gorilla Beta

horse Beta .................

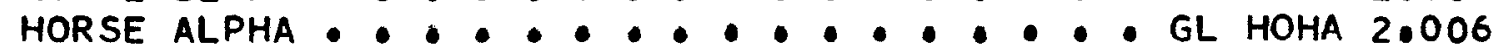

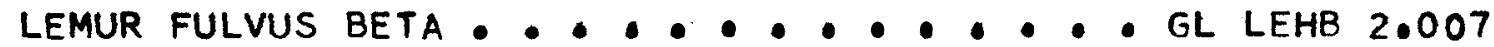

ABNORMAL HUMAN HEMOGLOBINS $\cdot . \cdot \cdot \cdot \cdot \cdot$. $\cdot$ GL HUH 2.020

MYOGLOBINS 2.100

WHALE -................... GL WHMY 2.101

II • OTHER RESPIRATORY PROTEINS 3.000

DIHEME PEPTIDE - CHROMATIUM • $\bullet \cdot \bullet \cdot \bullet \cdot-$ DH CH 3.001

FERREDOXIN - CLOSTRIDIUM PASTEURIANUM • • . FE CP 3.002

AZURIN - PSEUDOMONAS. ............ AZ PS 3.003

IV. RIBONUCLEASE $\quad 4.000$

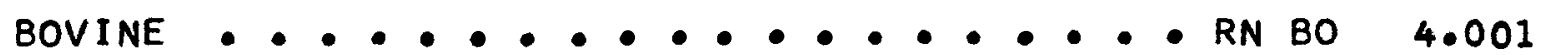

V. INHIBITORS

5.000

TRYPSIN INHIBITOR - BOVINE. • • • • • - TI BOPA 5.001 
PAGE

VI. TOBACCO MOSAIC VIRUS

6.000

TOBACCO MOSAIC VIRUS $\bullet \cdot \bullet \cdot \bullet \cdot \bullet \cdot \bullet$ TM TM 6.001 TOBACCO MOSAIC VIRUS DAHLMENSE. $\bullet \bullet . \bullet .$. TM TMD 6.002

VII. DIGESTIVE ENZYMES

7.000

CHYMOTRYPSINOGEN-A - BOVINE $\bullet \bullet \bullet \bullet \bullet \bullet-$ TR BOCH 7.001

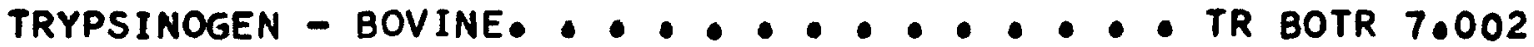

PAPAIN. $\bullet \bullet \bullet \bullet \bullet \bullet \bullet \bullet \bullet \bullet \bullet \bullet \bullet \bullet \bullet \bullet-$ PA PA 7.101

LYSOZYME - CHICKEN. $\bullet \bullet \bullet \bullet \bullet \bullet \bullet \bullet \bullet \bullet-L S$ CH 7.201

VIII - HORMONES

8.000

GLUCAGON

BOVINE $\bullet \cdot \cdot \cdot \cdot \cdot \cdot \cdot \cdot \cdot \cdot \cdot \cdot \cdot \cdot \cdot \cdot \cdot \cdot \cdot$ GN BO 8.001

PRESSINS

VASOPRESSIN ARGININE $\bullet \bullet \cdot \bullet \cdot \bullet \cdot-\bullet \cdot$ PR BOAR 8.101

VASOPRESSIN LYSINE $\bullet \bullet \cdot \bullet \cdot \bullet \cdot \bullet \bullet, \bullet \cdot$ PR PGLS 8.102

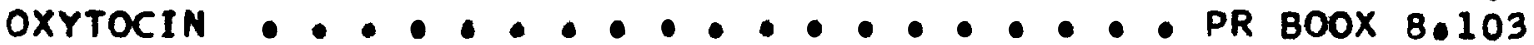

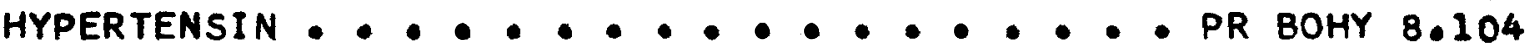

\section{CORTICOIDS}

ALPHA MELANOCYTE-STIMULATING HORMONE

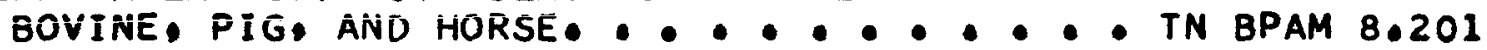

BETA MELANOCYTE-STIMULATING HORMONE

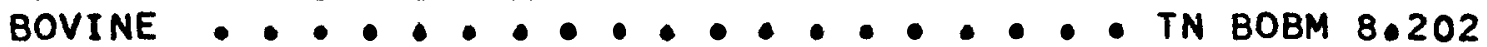

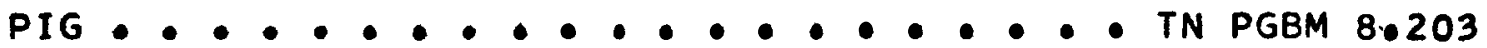

HORSE $\bullet \cdot \bullet \cdot \bullet \cdot \bullet \cdot \bullet \cdot \bullet \cdot \bullet \cdot \bullet \cdot \bullet$ TN HOBM 8.204

HUMAN ..................... TN HUBM 8.205

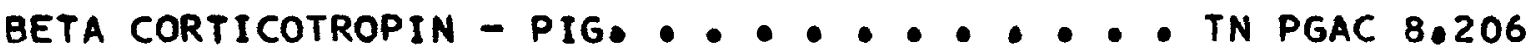

ALPHA CORTICOTROPIN - SHEEP AND BOVINE. $\bullet$. TN SBAC 8.207

INSULINS

INSULIN A

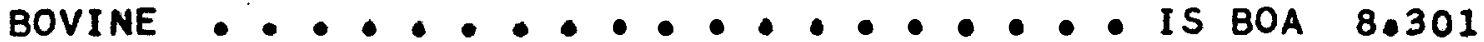

BONITO. $\bullet \cdot \bullet \cdot \bullet \cdot \bullet \cdot \bullet \cdot \bullet \cdot \bullet$ IS BNA 8.302

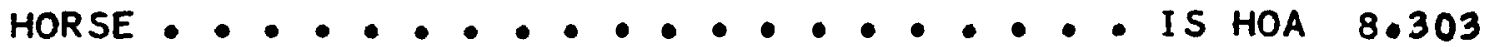

SHEEP . $\bullet \cdot \bullet \cdot \bullet \cdot \bullet \cdot \bullet \cdot \bullet \cdot \bullet \cdot$ - $\cdot$ IS SHA 8.304

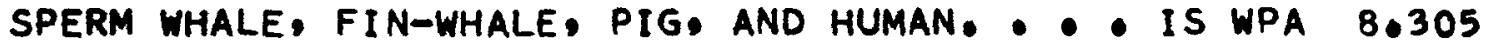

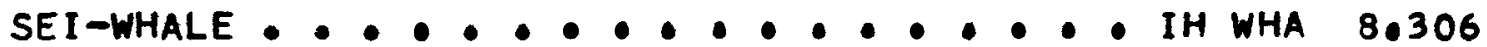

INSULIN B

BOVINE, SHEEP, HORSE, HUMAN, PIG, AND

SPERM WHALE. - $\bullet$ IS BOB 8.321

BONITO $\bullet \bullet \cdot \bullet \bullet \bullet \bullet \bullet \bullet \bullet \bullet \bullet \bullet \bullet \bullet \bullet \bullet \bullet$ IS BNB 8.322 
PAGE

IX. Plasma proteins

9.000

FIBRINOPEPTIDE A

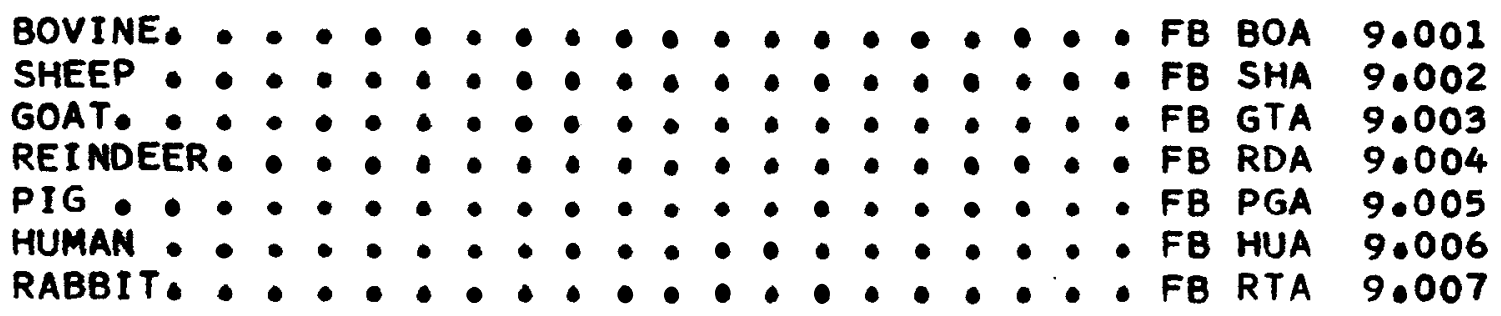

FIBRINOPEPTIDE B

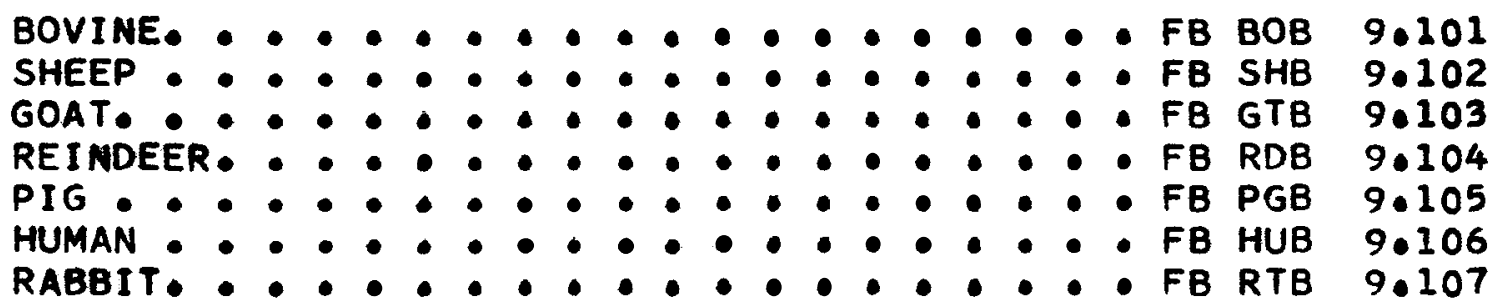

x Globulins

10.000

BENCE-JONES .................. BJ HU 10.001 
the meaning of the punctuation is as follows.

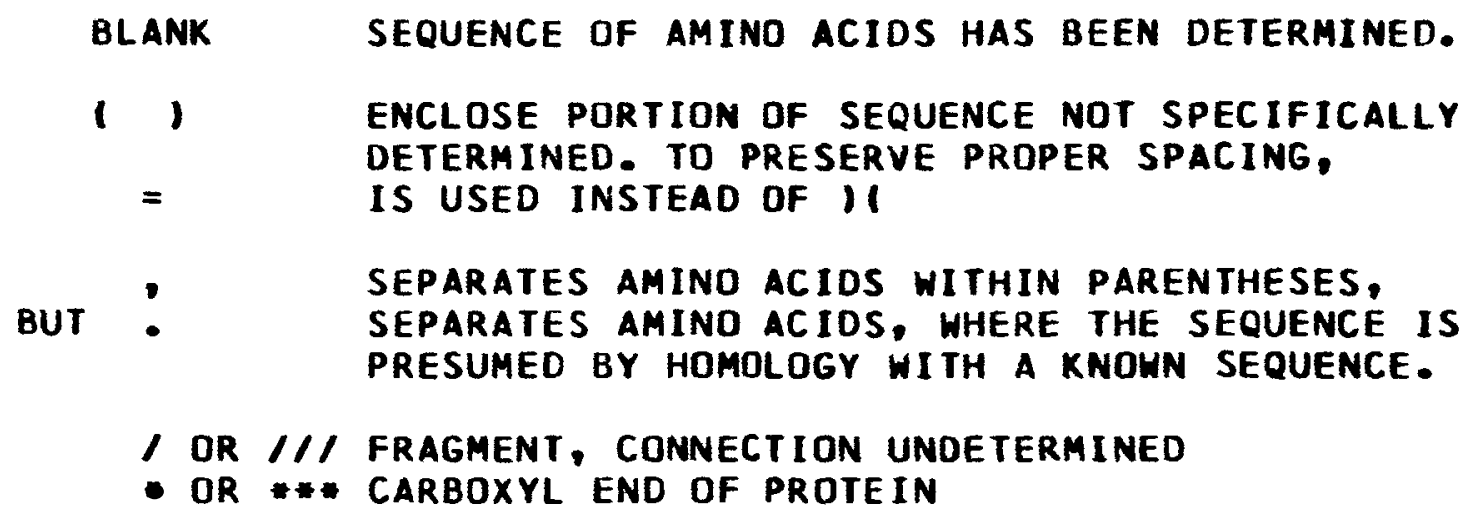

- ASTERISK BEFORE REFERENCE INDICATES THAT THE SEQUENCE HAS COPIED FROM, AND PROOFREAD AGAINST, THE ORIGINAL ARTICLE.

= BEFORE REFERENCE INDICATES THAT HE HAVE NOT SEEN THE ORIGINAL ARTICLE.

NO MARK BEFORE REFERENCE INDICATES OTHER GROUPS WHICH HAVE ALSO REPORTED hORK ON THE SAME PROTEIN. 
BOTH SINGLE- AND THREE-LETTER NOTATIONS ARE USED, AS FOLLOWS.

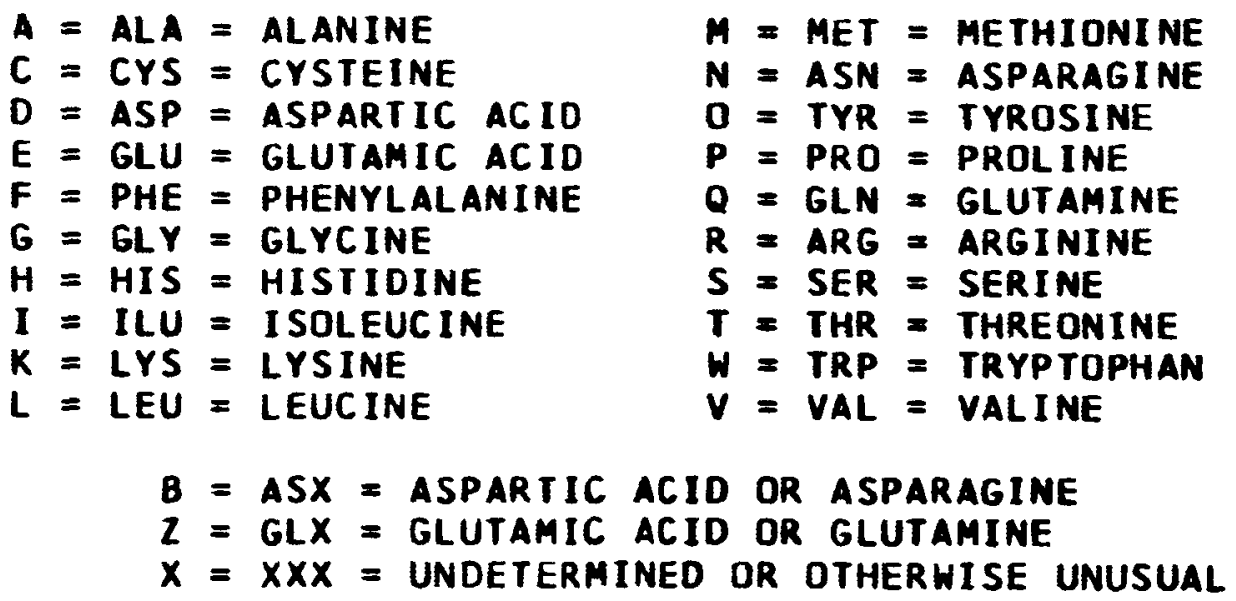

MNEMONICS OF THE ONE-LETTER CODE

IF POSSIBLE, THE INITIAL LETTER OF THE AMINO ACID IS USED. IF MOPE THAN GNE AMINO ACID BEgINS WITH THE SAME LETTER, THE MOST COMMONLY-OCCURRING ONE IS ASSIGNEO THE INITIAL.
$A=$ AL ANINE
$I$ = ISOLEUCINE
$S=$ SERINE
$C=$ CYSTEINE
$L=$ LEUCINE
$G=$ GLYCINE
$M=$ METHIONINE
$T=$ THREONINE
$H=$ HISTIDINE
$P=$ PROLINE
$v=$ VALINE

SOME OF THE DTHERS ARE PHONETICALLY SUgGESTIVE.

$$
\begin{aligned}
& F=\text { PHENYLALANINE } \\
& R=\text { ARGININE } \\
& 0=\text { TYROSINE }
\end{aligned}
$$

DOUBLE RING IN THE SIDE CHAIN.

$$
W=\text { TRYPTOPHAN }
$$

THE TWO ACIDS ARE ADJACENT, IN ALPHABETICAL ORDER.

$$
\begin{aligned}
& D=\text { ASPARTIC ACID } \\
& E=\text { GLUTAMIC ACID }
\end{aligned}
$$

the two amines have letters from the middle of the alphabet.

$$
\begin{aligned}
& N=\text { ASPARAGINE (CONTAINS N) } \\
& Q=\text { GLUTAMINE ('Q-TAMINE })
\end{aligned}
$$

NON-INITIAL LETTER AS CLOSE AS POSSIBLE TO ITS INITIAL.

$$
K=\text { LYSINE }
$$


CY TOCHROME C - BAKER IS YEAST

HEME BONDED TO CYSTEINES AT POSITIONS 19 AND 22.

123456789012345678901234567890

1 TEFKAGSAKKGATLFKTRCELCHTVEKG GP 31 HKVGPNLHGIFGRHSGOAQGOSOTTDANIKK 61 NVLWDENNMSEOLTNPKKOIPGTKMAFGGL 91 KKEKDRNDLITOLKKACE.

$\begin{array}{lllllllllllllll}1 & 2 & 3 & 4 & 5 & 6 & 7 & 8 & 9 & 10 & 11 & 12 & 13 & 14 & 15\end{array}$

1 thr glu phe lys ala gly ser ala lys lys gly ala thr leu phe LYS THR ARG CYS GLU LEU CYS HIS THR YAL GLU LYS GLY GLY PRO 31 HIS LYS VAL GLY PRO ASN LEU HIS GLY ILU PHE GLY ARG HIS SER GLY GLN ALA GLN GLY TYR SER TYR THR ASP ALA ASN ILU LYS LYS 61 ASN VAL LEU TRP ASP GLU ASN ASN MET SER GLU TYR LEU THR ASN PRO LYS LYS TYR ILU PRO GLY THR LYS MET ALA PHE GLY GLY LEU 91 LYS LYS GLU LYS ASP ARG ASN ASP LEU ILU THR TYR LEU LYS LYS ALA CYS GLU ***

COMPOSITION

\begin{tabular}{|c|c|c|c|c|c|c|c|c|c|c|}
\hline 7 & ALA & A & 2 & GLN & $Q$ & 8 & LEU & $\mathbf{L}$ & 4 & SER \\
\hline 3 & ARG & $\mathbf{R}$ & 7 & GLU & $\mathbf{E}$ & 16 & LYS & $K$ & 8 & THR \\
\hline 7 & ASN & $\mathbf{N}$ & 12 & GLY & $\mathbf{G}$ & 2 & MET & M & 1 & TRP \\
\hline 4 & ASP & D & 4 & HIS & $\mathbf{H}$ & 4 & PHE & $F$ & 5 & IYR \\
\hline 3 & CYS & C & 4 & ILU & $I$ & 4 & PRO & $\mathbf{P}$ & 3 & VAL \\
\hline
\end{tabular}

- NARITA,K.,TITANI, K.,YAOI, Y., MURAKAMI,H., BIOCHIM. BIOPHYS. ACTA, VOL. 77, PP.688-690, 1963 
CYTOCHROME C - CHICKEN

ACETYL AT AMINO END.

HEME BONDED TO CYSTEINES AT POSITIONS 14 AND 17.

123456789012345678901234567890

16 DIEKGKKIFVOKCSOCHTVEKGGKHKTGP

31 NLHGLFGRKTGOAEGESOTDANKNKGITHG 61 EDTLMEOLENPKKOIPGTKMIFAGIKKKSE 91 R V D LIADLKKATNS.

$\begin{array}{lllllllllllllll}1 & 2 & 3 & 4 & 5 & 6 & 7 & 8 & 9 & 10 & 11 & 12 & 13 & 14 & 15\end{array}$

1 GLY ASP ILU GLU LYS GLY LYS LYS ILU PHE VAL GLN LYS CYS SER GLN CYS HIS THR VAL GLU LYS GLY GLY LYS HIS LYS THR GLY PRO

31 ASN LEU HIS GLY LEU PHE GLY ARG LYS THR GLY GLN ALA GLU GLY PHE SER TYR THR ASP ALA ASN LYS ASN LYS GLY ILU THR TRP GLY

61 GLU ASP THR LEU MET GLU TYR LEU GLU ASN PRO LYS LYS TYR ILU PRo gly thr LYS MET ILU PHE ALA GLY ILU LYS LYS LYS SER GLU

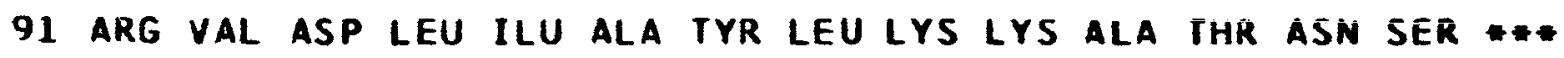

\section{COMPOSITION}

\begin{tabular}{|c|c|c|c|c|c|c|c|c|c|c|}
\hline 5 & ALA & $\mathbf{A}$ & 3 & GLN & $\mathbf{Q}$ & 6 & LEU & $\mathbf{L}$ & 4 & SER \\
\hline 2 & ARG & $\mathbf{R}$ & 7 & GLU & $\mathbf{E}$ & 18 & LYS & $K$ & 8 & THR \\
\hline & ASN & $\mathbf{N}$ & 13 & GLY & G & 2 & MET & $\mathbf{H}$ & 1 & TRP \\
\hline & ASP & D & 3 & HIS & H & 4 & PHE & $F$ & 4 & TYR \\
\hline & CYS & C & 7 & ILU & $\mathbf{I}$ & 3 & PRO & $\mathbf{P}$ & 3 & VAL \\
\hline
\end{tabular}

- MaRgol I ASH,E.,NEEDLEMAN,S.B. AND STEWART,J.H., aCTA CHEM. SCAND., VOL.17, SUPPL.1, PP.250-256, 1963 
CYTOCHROHE C - HORSE

ACETYL AT AMINO END.

HEME BONDED TO CYSTEINES AT POSITIONS 14 AND 17.

OXIDATION-REDUCTION POTENTIAL EQUALS . $250 \mathrm{~V}$.

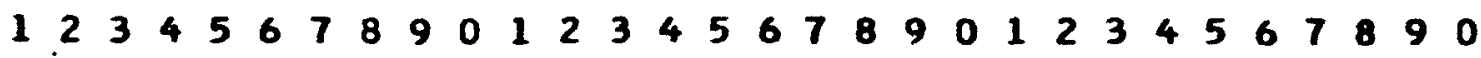

I G DVEKGKKIFVOKCAOCHTVEKGGKHKTG P

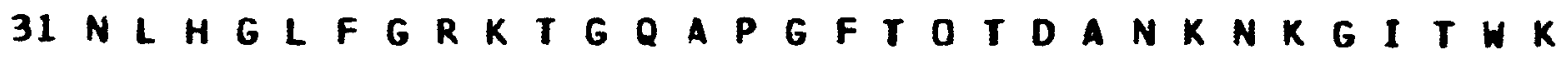

61 E E T L EOLEN PKK DI PG TKMIFAGIKKKTE

91 REDL I A OLKKA T NE*

$\begin{array}{lllllllllllllll}1 & 2 & 3 & 4 & 5 & 6 & 7 & 8 & 9 & 10 & 11 & 12 & 13 & 14 & 15\end{array}$

1 GLY ASP VAL GLU LYS GLY LYS LYS ILU PHE VAL GLN LYS CYS ALA GLN CYS HIS IHR VAL GLU LYS GLY GLY LYS HIS LYS THR GLY PRO

31 ASN IEU HIS GIY LEU PHE GIY ARE LYS THR ELY GIN ALA PRO GLY PHE THR TYR THR ASP ALA ASN LYS ASN LYS GLY ILU THR TRP LYS

61 GLU GLU THR LEU MET GLU TYR LEU GLU ASN PRO LYS LYS TYR ILU PRO GLY THR LYS MET ILU PHE ALA GLY ILU LYS LYS LYS THR GLU 91 arg glu ASP LEU ILU ALA TyR LEU LyS LYS ALA thr ASN GLU ***

\section{COMPOSITION}

\begin{tabular}{|c|c|c|c|c|c|c|c|c|c|c|}
\hline 6 & ALA & A & 3 & GLN & 0 & 6 & LEU & $L$ & 0 & SER \\
\hline 2 & ARG & $\mathbf{R}$ & 9 & GLU & $\mathbf{E}$ & 19 & LYS & $k$ & 10 & THR \\
\hline 5 & ASN & $\mathbf{N}$ & 12 & GLY & $\mathbf{G}$ & 2 & MET & $M$ & 1 & TRP \\
\hline 3 & ASP & D & 3 & HIS & $H$ & 4 & PHE & $F$ & 4 & TYR \\
\hline 2 & CYS & C & 6 & ILU & $\mathbf{I}$ & 4 & PRO & $\mathbf{P}$ & 3 & VAL \\
\hline
\end{tabular}

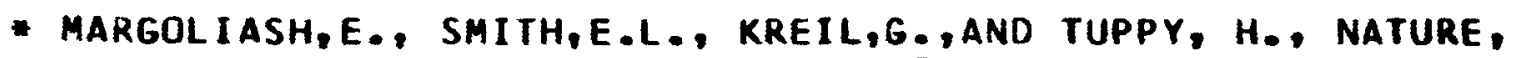
VOL.192, NO.4808, PP.1121-1127, DEC.23, 1961 
CYTOCHROME C - HUMAN

ACETYL AT AMINO END.

HEME BONDED TO CYSTEINES AT POSITIONS 14 AND 17.

LEU (L) REPLACES MET (M) AT POSITION 65 IN 10 PERCENT

YIELD IN POOLED PROTEIN.

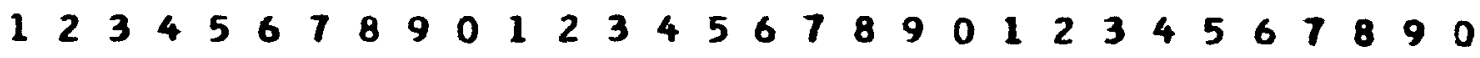

I G DVEKGKKIFIMKCSQCHTVEKGGKHKTE P

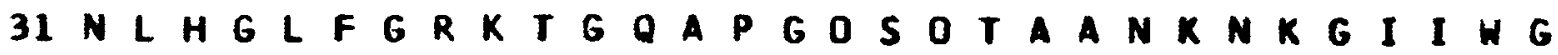
61 E D T L M ELEN PKKDIPG TKM IF V G IKKKEE 91 R A D L I OLKKA TNE

$\begin{array}{lllllllllllllll}1 & 2 & 3 & 4 & 5 & 6 & 7 & 8 & 9 & 10 & 11 & 12 & 13 & 14 & 15\end{array}$

1 gLY ASP VAL GLU LYS GLY LYS LYS ILU PHE ILU MET LYS CYS SER GLN CYS HIS THR VAL GLU LYS GLY GLY LYS HIS LYS THR GLY PRO

31 ASN LEU HIS GLY LEU PHE GLY ARG LYS THR GLY GLN ALA PRO GLY TYR SER TYR THR ALA ALA ASN LYS ASN LYS GLY ILU ILU TRP GLY 61 GLU ASP THR LEU MET GLU TYR LEU GLU ASN PRO LYS LYS TYR ILU PRD GLY THR LYS MET ILU PHE VAL GLY ILU LYS LYS LYS GLU GLU

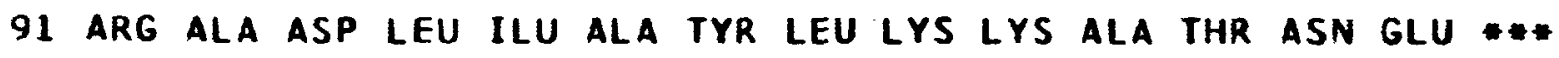

\section{COMPOSITION}

\begin{tabular}{|c|c|c|c|c|c|c|c|c|c|c|}
\hline 6 & ALA & A & 2 & GLN & 0 & 6 & LEU & $L$ & 2 & SER \\
\hline 2 & ARG & $\mathbf{R}$ & 8 & GLU & $\mathbf{E}$ & 18 & LYS & $\mathbf{k}$ & 7 & THR \\
\hline 5 & ASN & $\mathbf{N}$ & 13 & GLY & $\mathbf{G}$ & 3 & MET & $\mathbf{M}$ & 1 & TRP \\
\hline 3 & ASP & D & 3 & HIS & $H$ & 3 & PHE & $F$ & 5 & TYR \\
\hline 2 & CYS & C & 8 & ILU & I & 4 & PRD & $\mathbf{P}$ & 3 & VAL \\
\hline
\end{tabular}

- matsubara, H., AND SMITH,E.l., J. BIOL. ChEM., VOL. 237, NO.11, PC 3575-PC 3576, NOV. 1962 
CYTOCHROME - C PIG ANO BOVINE

ACETYL AT AMINO END.

HEME BONDED TO CYSTEINES AT POSITIONS 14 AND 17.

$\begin{array}{llllllllllllllllllllllllllllll} & 2 & 3 & 4 & 5 & 6 & 7 & 8 & 9 & 0 & 1 & 2 & 3 & 4 & 5 & 6 & 7 & 9 & 0 & 1 & 2 & 3 & 4 & 5 & 6 & 7 & 9 & 9\end{array}$

I G DVEKGKKIIFVQKCA OCH TVEKGGKHKT GP

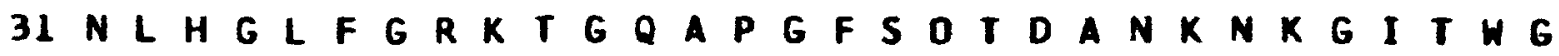
61 E E TLMEOLEN PKKOIPGIKMIFAG IKKKGE 91 R E L I A O LKKATNE.

$\begin{array}{lllllllllllllll}1 & 2 & 3 & 4 & 5 & 6 & 7 & 8 & 9 & 10 & 11 & 12 & 13 & 14 & 15\end{array}$

1 gLY ASP VAL GLU LYS GLY LYS LYS ILU PHE VAL GLN LYS CYS ALA GLN CYS HIS THR VAL GLU LYS GLY GLY LYS HIS LYS THR GLY PRO 31 ASN LEU HIS GLY LEU PHE GLY ARG LYS THR GLY GLN ALA PRO GLY PHE SER TYR THR ASP ALA ASN LYS ASN LYS GLY ILU THR TRP GLY 61 GLU GLU THR LEU MET GLU TYR LEU GLU ASN PRO LYS LYS TYR ILU PRO GLY THR LYS MET ILU PHE ALA GLY ILU LYS LYS LYS GLY GLU 91 ARg GLU ASP LEU ILU ALA TYR LEU LYS LYS ALA THR ASN GLU ***

\section{COMPOSI TION}

\begin{tabular}{|c|c|c|c|c|c|c|c|c|c|c|}
\hline 6 & ALA & A & 3 & GLN & $Q$ & 6 & LEU & $\mathbf{L}$ & 1 & SER \\
\hline 2 & ARG & $\mathbf{R}$ & 9 & GLU & $E$ & 18 & LYS & $K$ & 8 & THR \\
\hline 5 & ASN & $\mathbf{N}$ & 14 & GLY & $\mathbf{G}$ & 2 & MET & $M$ & 1 & TRP \\
\hline 3 & ASP & D & 3 & HIS & $\mathbf{H}$ & 4 & PHE & $F$ & 4 & TYR \\
\hline 2 & CYS & C & 6 & ILU & I & 4 & PRO & $\mathbf{P}$ & 3 & VAL \\
\hline
\end{tabular}

TOTAL NO. OF ACIDS $=104$

- margol iash, E. NEedleman, S.B. and Stehart, J.h., aCta Chem. SCAND.. VOL.17, SUPPL.1, PP.250-256, 1963 (PIG) 
CY PG $1.005-2$

TUPPY H., AND PALEUS, S., ACTA CHEM. SCAND., VOL. 13, NO.4. PP. 641-646, 1959, (HEME ATTACHEMENT REgION ONLY - BOVINE)

YASUNOBU, K. T., NAKASHIMA, T., HIGA, H., MATSUbaRA, H., AND BENSON, A. BIOCHIM. BIOPHYS. ACTA VOL. 78, PNI324 PP. 791-794, 1963 (BOVINE) 
CYTOCHROME C - PSEUDOMONAS

HEME BONDED TO CYSTEINES AT POSITIONS 12 AND 15.

THE AMINO END IS NOT ACETYLATED.

123456789012345678901234567890

I EDPEVLFKNKGCVACHAIDTKMVGPAOKDV 31 A AKFAG QAGAEAELAQRIKNGSQGVWGPIP 61 M P P A VS D DEAOTLAKWVLSQK.

$\begin{array}{lllllllllllllll}1 & 2 & 3 & 4 & 5 & 6 & 7 & 8 & 9 & 10 & 11 & 12 & 13 & 14 & 15\end{array}$

1 gLU ASP PRo GLU VAL LEU PHE LYS ASN LYS GLY CYS VAL aLA CyS HIS ALA ILU ASP THR LYS MET VAL GLY PRO ALA TYR LYS ASP VAL

31 ala ala lys phe ala gly gln ala gly ala glu ala glu leu ala GLN ARG ILU LYS ASN GLY SER GLN GLY VAL TRP GLY PRO ILU PRO 61 MET PRo PRo ASN ALA YAL SER ASP ASP GLU ALA GLî THK LEU aLA LYS TRP VAL LEU SER GLN LYS **

\section{COMPOSITION}

\begin{tabular}{|c|c|c|c|c|c|c|c|c|c|c|}
\hline 3 & ALA & A & 5 & GLN & $Q$ & 4 & LEU & $L$ & 3 & SER \\
\hline 1 & ARG & $\mathbf{R}$ & 5 & GLU & $E$ & 8 & LYS & $K$. & 2 & THR \\
\hline 3 & ASN & $\mathbf{N}$ & 7 & GLY & $\mathbf{G}$ & 2 & MET & $\mathbf{M}$ & 2 & TRP \\
\hline 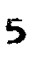 & ASP & D & 1 & HIS & $\mathbf{H}$ & 2 & PHE & $\mathbf{F}$ & 1 & TYR \\
\hline 2 & CYS & C & 3 & ILU & $\mathbf{I}$ & 6 & PRO & $p$ & 7 & VAL \\
\hline
\end{tabular}

- AMBLER, R. P., BIOCHEM. J., VOL.89, P.349-378. 1963 
CYTOCHROME C - TUNA FISH

ACETYL AT AMINO END.

HEME BONDED TO CYSTEINES AT POSITIONS 14 AND 17.

1234567890123456789012345678990

1 G DVA K.G K K.TFV O K.C A QIC.HIT VENG G K.H KIV.G.P.

31 NIL H.G L F.G R.K TIG.QIA E G D.S O TID.A.NIK.S K.G I V WIN.

61 N.DIT L M E O.LEN PKK.OII.P.GITKIM.IIF.A G I KK.KGE

91 R.Q D LIV.A)O.LKSTAS

$\begin{array}{lllllllllllllll}1 & 2 & 3 & 4 & 5 & 6 & 7 & 8 & 9 & 10 & 11 & 12 & 13 & 14 & 15\end{array}$

1 GLY ASP VAL ALA LYS.GLY LYS LYS.THR PHE VAL GLN LYS.CYS ALA GLNICYS.HISITHR VAL GLU ASN GLY GLY LYS.HIS LYSIYAL.GLY.PRO.

31 ASNILEU TRP.GLY LEU PHE.GLY ARG.LYS THR(GLY.GLN)ALA GLU GLY TYR.SER TYR THR(ASP.ALA.ASN)LYS.SER LYS.GLY ILU VAL TRPIASN,

61 ASN,ASPITHR LEU MET GLU TYR.LEU GLU ASN PRO LYS LYS.TYRIILU. PRO.GLYITHR LYS.MET. ILUJPHE.ALA GLY ILU LYS LYS.LYS GLY GLU 91 ARG.GLN ASP LEUIVAL. ALAITYR.LEU LYS SER THR ALA SER ***

\section{COMPOSITION}

\begin{tabular}{|c|c|c|c|c|c|c|c|c|c|c|}
\hline 7 & ALA & A & 4 & GLN & $Q$ & 6 & LEU & $\mathbf{L}$ & 4 & SER \\
\hline 2 & ARG & $\mathbf{R}$ & 5 & GLU & $\mathbf{E}$ & 16 & LYS & $K$ & 7 & THR \\
\hline 6 & ASN & $N$ & 13 & GLY & $\mathbf{G}$ & 2 & MET & $\mathbf{M}$ & 2 & TRP \\
\hline 4 & ASP & D & 2 & HIS & $H$ & 3 & PHE & $\mathbf{F}$ & 5 & TYR \\
\hline 2 & CYS & $C$ & 4 & ILU & $I$ & 3 & PRD & $\mathbf{P}$ & 6 & VAL \\
\hline
\end{tabular}

TOTAL ND. OF ACIDS $=103$

* KREIL,G., 2. PHYStol. CHEM., BD. 334, PP.154-166, 1963 
CYTOCHROME C - BOMBYX MORI (SILKWORM)

HEME BONDED TO CYSTEINES AT POSITIONS 4 AND 7 OF FRAGMENT.

123456789901

I VORCAQCH T(V,E)I

$\begin{array}{lllllllllll}1 & 2 & 3 & 4 & 5 & 6 & 7 & 8 & 9 & 10 & 11\end{array}$

// VAL GLN ARG CYS ALA GLN CYS HIS THR(VAL,GLU)//

COMPOSITION OF FRAGMENT

\begin{tabular}{|c|c|c|c|c|c|c|c|c|c|c|}
\hline 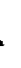 & ALA & A & 2 & GLN & $Q$ & 0 & LEU & $\mathrm{L}$ & 0 & SER \\
\hline$l$ & ARG & $\mathbf{R}$ & 1 & GLU & $E$ & 0 & LYS & $K$ & 1 & THR \\
\hline 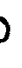 & ASN & $N$ & 0 & GLY & $\mathbf{G}$ & 0 & MET & $M$ & 0 & TRP \\
\hline 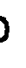 & ASP & D & 1 & HIS & $\mathrm{H}$ & 0 & PHE & $\mathbf{F}$ & 0 & TYR \\
\hline & CYS & $c$ & 0 & $\underline{I L U}$ & $\mathbf{I}$ & 0 & PRO & $\mathbf{P}$ & 2 & VAL \\
\hline
\end{tabular}

* TUPPY H., Z. NATURFORSCH,, VOL.12, PP.784-788, 1957 
CYTOCHROME C - RATTLESNAKE

ACETYL AT AMINO END.

123456789012345678901234567890

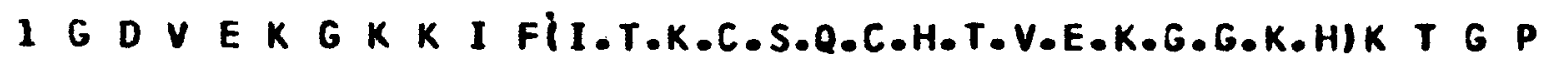

31 N L H.G L F.G RK T G Q A V G D.S O.TA A N K N.K G I I H.G G1 D D T L M E O.L EN PKK O.IP G TK M.V F.T G L.SKKKE 91 R T N L.I A D.LKEKTAA -

$\begin{array}{lllllllllllllll}1 & 2 & 3 & 4 & 5 & 6 & 7 & 8 & 9 & 10 & 11 & 12 & 13 & 14 & 15\end{array}$

1 GLY ASP VAL GLU LYS GLY LYS LYS ILU PHEIILU.THR.LYS.CYS.SER. GLN.CYS.HIS.THR.VAL.GLU.LYS.GLY.GLY.LYS.HISILYS THR GLY PRO

31 ASN LEU HIS.GLY LEU PHE.GLY ARG LYS THR GLY GLN ALA VAL GLY TYR.SER TYR.THR ALA ALA ASN LYS ASN.LYS GLY ILU ILU TRP.GLY

61 ASP ASP THR LEU MET GLU TYR.LEU GLU ASN PRO LYS LYS TYR.ILU PRD GLY THR LYS MET.VAL PHE.THR GLY LEU.SER LYS LYS LYS GLU

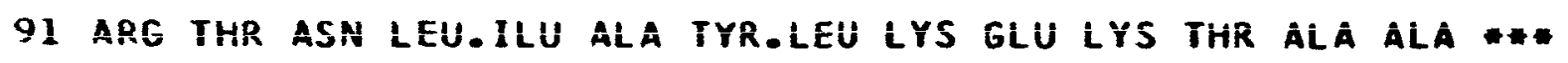

\section{COMPOSITION}

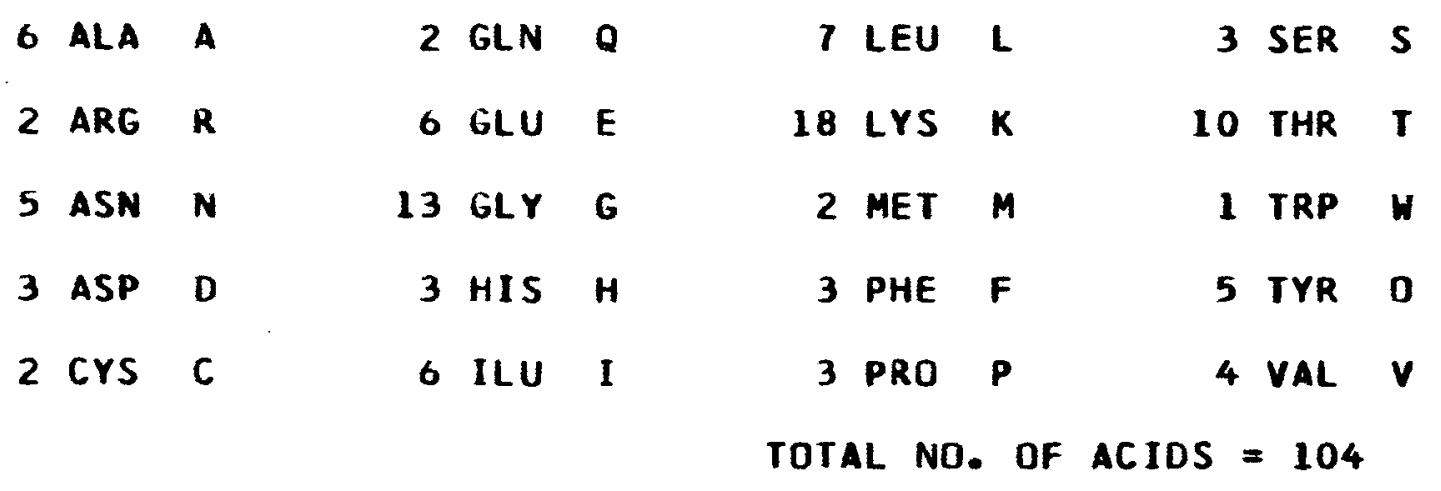

- BAHL, D. P. AND SMITH, E. L., J. BIOL. CHEM., VOL.240, NO.9, PP.3585-3593, SEPT., 1965 
CYTOCHROME - C RHODOSPIR ILLUM RUBRUM

HEME BONDED TO CYSTEINES AT POSITIONS 1 AND 4 OF FRAGMENT.

1234567890123

I CLACHTFBZGANKI

$\begin{array}{lllllllllllll}1 & 2 & 3 & 4 & 5 & 6 & 7 & 8 & 9 & 10 & 11 & 12 & 13\end{array}$

// CYS LEU ALA CYS HIS THR PHE ASX GLX GLY ALA ASN LYS $/ /$

COMPOSITION OF FRAGMENT

\begin{tabular}{|c|c|c|c|c|c|c|c|c|c|c|}
\hline 2 & ALA & A & 0 & GLN & $a$ & 1 & LEU & L & 0 & SER \\
\hline 0 & ARG & $\mathbf{R}$ & 0 & GLU & $E$ & 1 & LYS & $k$ & 1 & THR \\
\hline 1 & ASN & $\mathbf{N}$ & 1 & GLY & $\mathbf{G}$ & 0 & MET & $M$ & 0 & TRP \\
\hline 0 & ASP & $D$ & 1 & HIS & $\mathbf{H}$ & 1 & PHE & $F$ & 0 & TYR \\
\hline 2 & cys & $c$ & 0 & ILU & I & 0 & PRO & $p$ & 0 & VALL \\
\hline & ASX & B & 1 & $G L X$ & 2 & & & & & \\
\hline
\end{tabular}

- TUPPY H., and Paleus, S., ACTa CHEM. SCANO., Vol. 13, N0.4, PP. 641-646, 1959 
CYTOCHRDME C - SALMON

HEME BONDED TO CYSTEINES AT POSITIONS 4 AND 7 OF FRAGMENT.

$\begin{array}{lllllllllll}1 & 2 & 3 & 4 & 5 & 6 & 7 & 8 & 9 & 0 & 1\end{array}$

IVOKCAOHCT $(V, E) /$

$\begin{array}{lllllllllll}1 & 2 & 3 & 4 & 5 & 6 & 7 & 8 & 9 & 10 & 11\end{array}$

// VAL GLN LYS CYS ALA GLN CYS HIS THR (VAL, GLU)//

COMPOSITION OF FRAGMENT

\begin{tabular}{|c|c|c|c|c|c|c|c|c|c|}
\hline$\hat{A} \mathbf{L} \dot{A}$ & $\dot{\mathbf{A}}$ & 2 & GLiN & $\mathbf{2}$ & 0 & LEV & 1 & 0 & SER \\
\hline ARG & $\mathbf{R}$ & 1 & GLU & $E$ & 1 & LYS & $\mathbf{K}$ & 1 & THR \\
\hline ASN & $\mathbf{N}$ & 0 & GLY & $\mathbf{G}$ & 0 & MET & $M$ & 0 & TRP \\
\hline ASP & D & 1 & HIS & $\mathbf{H}$ & 0 & PHE & $F$ & 0 & TYR \\
\hline CYS & $C$ & 0 & ILU & I & 0 & PRO & $\mathbf{P}$ & 2 & VAL \\
\hline
\end{tabular}

* Tuppy H., and paleus, S., aCta CHem. SCAND., vol. 9, P. 353-364, 1955 
HEMOGLOBIN ALPHA - HUMAN

$\begin{array}{llllllllllllllllllllllllllllll}2 & 3 & 4 & 5 & 6 & 7 & 8 & 9 & 0 & 1 & 2 & 3 & 4 & 5 & 6 & 7 & 8 & 9 & 0 & 1 & 2 & 3 & 4 & 5 & 6 & 7 & 8 & 9 & 0\end{array}$

IVLS PADKTNVKAAHGKVGAHAGEOGAEALE 31 RFLSFPTIKTOFPHFDLSHGSA O VKGHGK 61KVA DALTNA VAHVDDHANALSALS DLHAH K 91 L R D P V NFKLLSHCLLVTLAAHLAAEFTA A 121 VHASLDKFLASVSTVLTSKOR *

$$
\begin{array}{lllllllllllllll}
1 & 2 & 3 & 4 & 5 & 6 & 7 & 8 & 9 & 10 & 11 & 12 & 13 & 14 & 15
\end{array}
$$

1 VAL LEU SER PRO ALA ASP LYS THR ASN VAL LYS ALA ALA TRP GLY LyS VAl gly ala his ala gly glu tYr gly ala glu ala leu glu 31 ARg MET PHE LEU SER PHE PRO THR THR LYS THR TYR PHE PRO HIS PHE ASP LEU SER HIS GLY SER ALA GLN VAL LYS GLY HIS GLY LYS

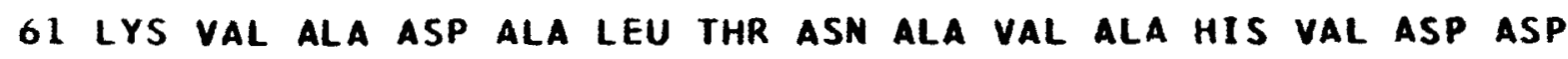
met pRo asn ala leu SER ala leu SER asp leu his ala his lys 91 LeU ARg VAL ASP PRO YAL ASN PHE LYS LEU LEU SER HIS CYS LEU leu val thr leu ala ala his leu pro ala glu phe thr pro ala 121 VAL hIS ALA SER LEU ASP LYS PHE LEU ALA SER VAL SER THR VAL LEU THR SER LYS TYR ARG *:*

COMPOSITION

\begin{tabular}{|c|c|c|c|c|c|c|c|c|c|c|}
\hline 21 & ALA & $\mathbf{A}$ & 1 & GLN & $Q$ & 18 & LEU & $\mathbf{L}$ & 11 & SER \\
\hline 3 & ARG & $\mathbf{R}$ & 4 & GLU & $\mathbf{E}$ & 11 & LYS & $K$ & 9 & THR \\
\hline 4 & ASN & $\mathbf{N}$ & 7 & GLY & $\mathbf{G}$ & 2 & MET & $M$ & 1 & TRP \\
\hline 8 & ASP & D & 10 & HIS & $H$ & 7 & PHE & $F$ & 3 & TYR \\
\hline 1 & CYS & $C$ & 0 & $I L U$ & 1 & 7 & PRO & $\mathbf{P}$ & 13 & VAL \\
\hline
\end{tabular}


- HILL, R.J.. AND KONIGSBERG, W., J. BIOL. CHEM., VOL. 237, Mo.10, PP. 3151-3156, OCT., 1962

BRAUNITZER, G., GEHRING-MULLER, R., HILSCHMANH, N., HILSE, K., HOBOM, G., RUDLOFF, V., AND WITTMANN-LIEBOLD, B...

2. PHYS IOL. CHEM., VOL. BD 325, PP.283-286, 1961

THE SAME SEQUENCE, hithout distinguishing AMINES, also

REPORTED IN THE ARTICLE.

SCHROEDER,W.A., J.R.SHELTON, J.B.SHELTON, AND J.CORMICK BIOCHEMISTRY, VOL. 2, NO.6, PP.1353-1357, NOV.-DEC., 1963

FETAL ALPHA CHAIN IS VERY PROBABLY IDENTICAL WITH ADULT ALPHA CHAIN. TRYPTIC AND CHYMOTRYPTIC PEPTIDES, MOST OF WHICH WERE COMPLETELY SEQUENCED, WERE SHOWN TO FIT EXACTLY INTO THE ADULT .ALPHA CHAIN SEQUENCE. 
HEMOGLOBIN BETA - HUMAN

$\begin{array}{llllllllllllllllllllllllllllll}1 & 2 & 3 & 4 & 5 & 6 & 7 & 8 & 9 & 0 & 1 & 2 & 3 & 4 & 5 & 6 & 7 & 9 & 9 & 1 & 2 & 3 & 4 & 5 & 6 & 7 & 8 & 9 & 0\end{array}$

IVHLTPEEKSAVTALWGKVDVDEVGGEALGR

31 L LVVOPHTERFFESFG DLSTPDAVM G D P K V 61 KAHGKKYLGAFSDGLAHLDDLKGTFATLSE 91 L CDKLHVDPEDFRLLGDVLVCVLAHHFGK 121 EF TP P VAA OEKVVAGVADALAHKOH

$$
\begin{array}{lllllllllllllll}
1 & 2 & 3 & 4 & 5 & 6 & 7 & 8 & 9 & 10 & 11 & 12 & 13 & 14 & 15
\end{array}
$$

I VAL HIS LEU THR PRO GLU GLU LYS SER ALA VAL THR ALA LEU TRP GLY LYS VAL ASP VAL ASP GLU VAL GLY GLY GLU ALA LEU GLY ARG

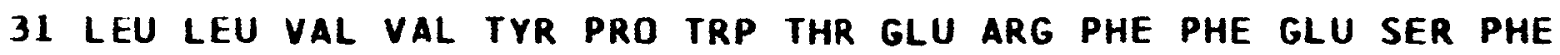
GLY ASP LEU SER THR PRO ASP ALA VAL MET GLY ASP PRO LYS VAL 61 LYS ALA HIS GLY LYS LYS VAL LEU GLY ALA PHE SER ASP GLY LEU ALA HIS LEU ASP ASP LEU LYS GLY THR PHE ALA THR LEU SER GLU 91 LEU HIS CYS ASP LYS LEU hIS VAL ASP PRO GLU ASP PHE ARg LeU LEU GLY asp VAL LEU VAL CYS VAL LEU ALA HIS hIS PHE GLY LyS izi glu phe thr pró pró val glu ala ala tró glu lys val val ala gly val ala asp ala leu aLA his lys tYR HIS ***

\section{COMPOSITION}

\begin{tabular}{|c|c|c|c|c|c|c|c|c|c|c|}
\hline 15 & ALA & $A$ & 0 & GLN & 0 & 18 & LEU & $\mathbf{L}$ & 5 & SER \\
\hline 3 & ARG & $R$ & 11 & GLU & $\mathbf{E}$ & 11 & LYS & $K$ & 7 & THR \\
\hline 0 & ASN & $\mathbf{N}$ & 13 & GLY & $\mathbf{G}$ & 1 & MET & $M$ & 2 & TRP \\
\hline 13 & ASP & D & 9 & HIS & $H$ & 8 & PHE & $F$ & 3 & TYR \\
\hline 2 & CYS & C & 0 & ILU & I & 7 & PRD & $\mathbf{P}$ & 18 & VAL \\
\hline
\end{tabular}

- BRAUNITZER, G., GEHRING-MULLER, R., HILSCHMANN, N., HILSE, K., HOBOM, G., RUDLOFF, $Y .$, AND HITTMANN-LIEBOLD, B.,

Z. PHYSIOL. CHEM., VOL. BD 325, PP.283-286, 1961 
HEMOGLOBIN GAMHA - HUMAN

123445677890122345677899012234567896

I GHF TEE DKATITSLHGKVNVEDAGGETLGR 31 L LVVDPWTORFFDSFGNLSSASA IMGN PKV 61 KA H GKKVLTS L G D A I KHLDODLKG TFA Q LSE $91 L H C D K L H V D P E N F K L L G N V L V T V L A I H F G K$ 121 EF T P EV Q A S UKMVTGVASALSS R DH*

$\begin{array}{lllllllllllllll}1 & 2 & 3 & 4 & 5 & 6 & 7 & 8 & 9 & 10 & 11 & 12 & 13 & 14 & 15\end{array}$

1 GLY HIS PHE THR gLU GLU ASP LYS ALA THR ILU THR SER LEU TRP GLY LYS VAL ASN VAL GLU ASP ALA GLY GLY GLU THR LEU GLY ARG

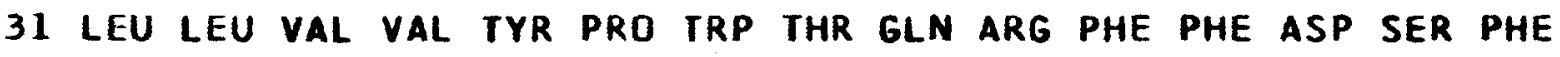
GLY ASN LEU SER SER ALA SER ALA ILU MET GLY ASN PRO LYS VAL 61 LYS ALA HIS GLY LYS LYS VAL LEU THR SER LEU GLY ASP ALA ILU LYS HIS LEU ASP ASP LEU LYS GLY THR PHE ALA GLN LEU SER GLU 91 LEU hIS CYS ASP LYS LEU hIS VAL ASP PRO gLU ASN PHE LYS LeU LEU GLY ASN VAL LEU VAL THR VAL LEU ALA ILU HIS PHE GLY LYS 121 GLU PHE THR PRO GLU VAL GLN ALA SER TRP GLN LYS MET VAL THR gly val ala ser ala leu ser ser arg tyr his *:*

COMPOSITION

\begin{tabular}{|c|c|c|c|c|c|c|c|c|c|c|}
\hline 11 & ALA & A & 4 & GLN & $\mathbf{Q}$ & 17 & LEU & $L$ & 11 & SER \\
\hline 3 & ARG & $\mathbf{R}$ & 8 & GLU & $E$ & 12 & LYS & $k$ & 10 & THR \\
\hline 5 & $\mathbf{A S N}$ & $\mathbf{N}$ & 13 & GLY & G & 2 & MET & $M$ & 3 & TRP \\
\hline 8 & ASP & D & 7 & HIS & $H$ & 8 & PHE & $F$ & 2 & TYR \\
\hline 1 & CYS & $c$ & 4 & ILU & I & 4 & PRD & $\mathbf{P}$ & 13 & VAL \\
\hline
\end{tabular}

- SCHROEDER, W.A., SHELTON, J.R., SHELTON, J.B., CORMICK, J., AND JONES,R.T., BIOCHEMISTRY, VOL.2, NO.5, PP.992-1008, SEPT.-OCT., 1963 
HEMOGLOBIN BETA - GORILLA

12345678901234567899012345678990

IVHLTPEEKSAVTALHGKVDVDEVGGEALGR $31 L L V V O P N T E R F F E S F G D L S T P D A V M G A N K V$ 61 KAHGKKVLGAFSDGLAHLDDLKGTFATLSE 91 L H DKLHVDPEDFLLLGDVLVCVLAHHFGK 121 EF TP P E A A D K V V A G A DALAHKOH.

$\begin{array}{lllllllllllllll}1 & 2 & 3 & 4 & 5 & 6 & 7 & 8 & 9 & 10 & 11 & 12 & 13 & 14 & 15\end{array}$

1 VAL HIS LEU THR PRO GLU GLU LYS SER ALA VAL THR ALA LEU TRP GLY LYS VAL ASP VAL ASP GLU VAL GLY GLY GLU ALA LEU GLY ARG

31 LeU leu val val tyr pro trp thr glu arg phe phe glu ser phe GLY ASP LEU SER THR PRD ASP ALA VAL MET GLY ASP PRO LYS VAL

61 LyS ALA hIS gLY LYS LYS VAL LEU gLY ALA PHE SER ASP gLY LEU ALA HIS LEU ASP ASP LEU LYS GLY THR PHE ALA THR LEU SER GLU

91 LEU hIS CYS ASP LYS LEU hIS VAL ASP PRO GLU ASP PHE LEU LEU

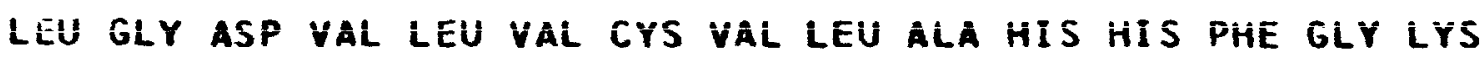
121 GLU PHE thr pRo pRo VAL GLU ALA ALA TYR GLU LyS VAL VAL aLA gly val ala asp ala leu ala his lys tyr his ***

COMPOSITION

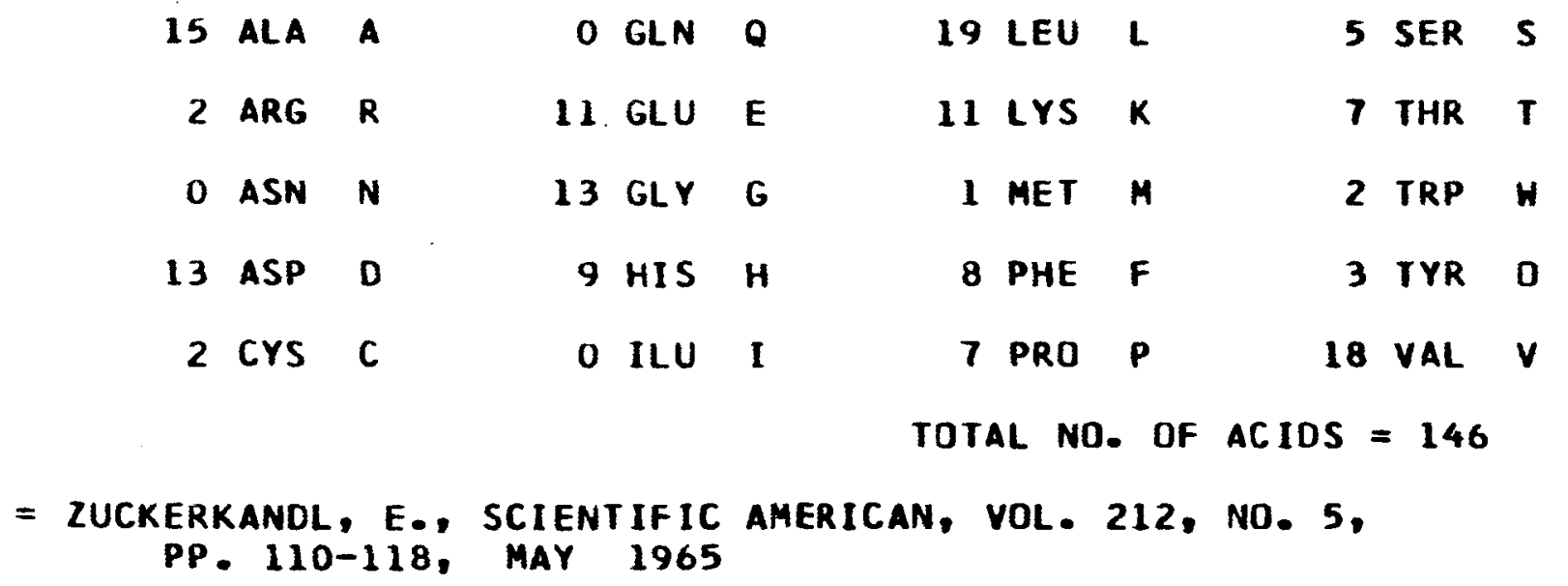


HEMOGLOBIN BETA - HORSE

123456789012345678901234567890

IVELS GEEKAALIV,A,L,H,D)K V DEE E G GIGEAA)LG R

31 L L V O P H T ER FIF.E.S.F.G.D.L.S.G.P.D.A.VIMIG.D.PIK V

61 KAHGKKVLLHSFGEGVHHIL.D.D.LIK G T F AIA.L.S.E.

91 L.H.C.D.K.L.H•V.D.P.E•D.FIR L L G D L A L V VARHFGK 121 DFT PELEASOEKVVAGVADALAHKOH*

$\begin{array}{llllllllllllllll}1 & 2 & 3 & 4 & 5 & 6 & 7 & 8 & 9 & 10 & 11 & 12 & 13 & 14 & 15\end{array}$

I VAL GLU LEU SER GLY GLU GLU LYS ALA ALA LEUIVAL, ALA,LEU, TRP, ASPILYS VAL ASP GLU GLU GLU VAL GLYIGLY.GLU.ALAILEU GLY ARG

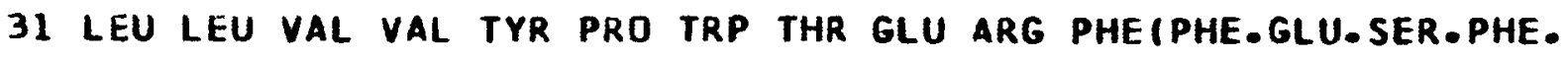
GLY.ASP.LEU.SER.GLY.PRO.ASP.ALA.VALIMETIGLY.ASP.PROILYS VAL

61 LYS ALA HIS GLY LYS LYS VAL LEU HIS SER PHE GLY GLU GLY VAL

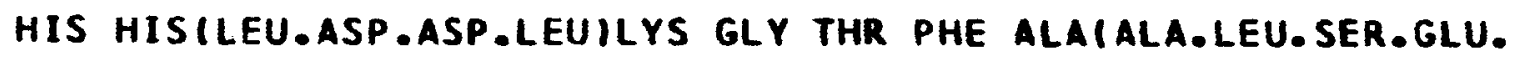

91 LEU.HIS.CYS.ASP.LYS.LEU.HIS.VAL.ASP.PRO.GLU.ASP.PHEIARG LEU LeU gly asp Val leu ala leu val val ala arg his phe gly lys 121 ASP PHE THR PRo GLU LEU GLU ALA SER TYR GLU LYS VAL VAL ALA gly val ala asp ala leu ala his lys tyr his ***

COMPOSITION

\begin{tabular}{|c|c|c|c|c|c|c|c|c|c|c|}
\hline 15 & ALA & A & 0 & GLN & 0 & 19 & LEU & $L$ & 6 & SER \\
\hline 4 & ARG & $\mathbf{R}$ & 15 & GLU & $\mathbf{E}$ & 11 & LYS & $\mathbf{k}$ & 3 & THR \\
\hline 0 & ASN & $\mathbf{N}$ & 14 & GLY & $\mathbf{G}$ & 1 & ME T & $M$ & 2 & TRP \\
\hline 13 & ASP & D & 9 & HIS & $\mathbf{H}$ & 8 & PHE & $\mathbf{F}$ & 3 & TYR \\
\hline 1 & CYS & $C$ & 0 & ILU & I & 5 & PRO & $\mathbf{P}$ & 17 & VAL \\
\hline
\end{tabular}

TOTAL NO. OF ACIDS $=146$

- SMITH, D. B.. CAN. J. BIOCHEM., VOL.42, N0.5, PP.755-762, 1964 
HEMOGLOBIN ALPHA - HORSE

$\begin{array}{lllllllllllllllllllllllllllllll}1 & 2 & 3 & 4 & 5 & 6 & 7 & 8 & 9 & 0 & 1 & 2 & 3 & 4 & 5 & 6 & 7 & 8 & 9 & 0 & 1 & 2 & 3 & 4 & 5 & 6 & 7 & 8 & 9 & 0\end{array}$

IVL S A A DKT N V KAAWSKVGGHAGEOGAEALE 31 R M L GFP T TK T DFPHFDLSHGSA Q VKAHGK 61KVA D GLTLAVGHLDDLPGALSNLSDLHAHK 91 L R D P V N K L L S H C L L S T L A V H L P N D F T PA 121 VHA S L D KFLS SVSTVLTSKOR*

$\begin{array}{lllllllllllllll}1 & 2 & 3 & 4 & 5 & 6 & 7 & 8 & 9 & 10 & 11 & 12 & 13 & 14 & 15\end{array}$

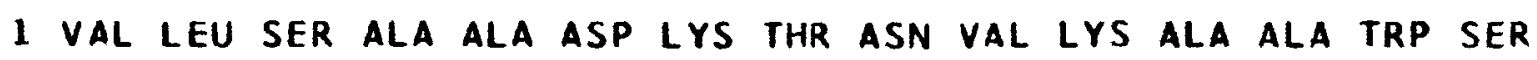

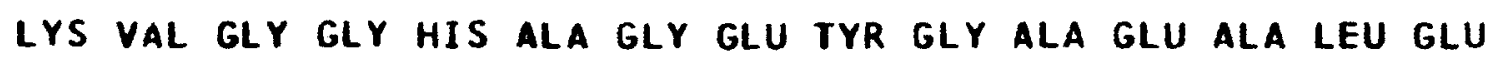
31 ARG MET PHE LEU GLY PHE PRO THR THR LYS THR TYR PHE PRO HIS PHe ASP LEU SER HIS GLY SER ALA GLN VAL LyS ALA HiS GLY LyS 61 LYS VAL ALA ASP GLY LEU THR LEU ALA VAL GLY HIS LEU ASP ASP

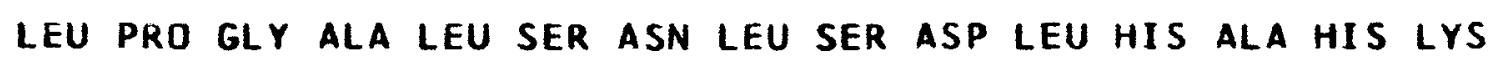
91 LeU ARg VAl ASP PRo VAL ASN PHE LYS LEU LEU SER HIS CYS LEU LEU SER THR LEU ALA VAL HIS LEU PRO ASN ASP PHE THP PRO ALA 121 VAL HIS ALA SER LEU ASP LYS PHE LEU SER SER VAL SER THR VAL LEU THR SER LYS TYR ARG ***

\section{COMPOSITION}

\begin{tabular}{|c|c|c|c|c|c|c|c|c|c|c|}
\hline 16 & ALA & $\mathbf{A}$ & 1 & GLN & $\mathbf{Q}$ & 21 & LEU & $L$ & 13 & SER \\
\hline 3 & ARG & $\mathbf{R}$ & 3 & GLU & $E$ & 11 & LYS & $K$ & 9 & THR \\
\hline 4 & ASN & $\mathbf{N}$ & 10 & GLY & $\mathbf{G}$ & 1 & MET & $M$ & 1 & TRP \\
\hline 9 & ASP & $\mathbf{D}$ & 10 & HIS & $H$ & 7 & PHE & $\mathbf{F}$ & 3 & TYR \\
\hline 1 & CYS & $c$ & 0 & ILU & I & 6 & PRD & $\mathbf{P}$ & 12 & VAL \\
\hline
\end{tabular}

TOTAL NO. OF ACIDS $=141$

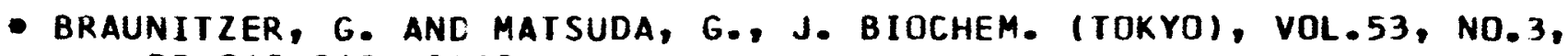
PP. 262-263, 1963

THIS SEQUENCE WAS DETERMINED PARTIALLY BY HOMOLOGY WITH HUMAN ALPHA. 
HEMOGLOBIN BETA - LEMUR FULVUS

1234567890123445678990112345678890

1 TLLSA EEDAHVTSLWGKVNVEKVGGEALGR

$31 L L V V \& O, P, H, T, E, R, F, F, E, S, F, G, D=L, S, S, P, S, A, V, H, G, D, P, K, V \bullet$

$61 K, A, H, G, K, K, V, L, S, A, F, S, E, G=L, H, H, L, D, D, L, K, G, T, F, A, A, L, S, E$,

$91 L, H, C, V, A, L, H, V, D, P, E, D, F, K, L, L, G, D, S, L, S, D, V, L, A, D, H, F, G, K)$

$121 \times \times \times \times \times \times \times \times \times \times \times \times \vee \vee A G \vee(A, D, A, L, A, H, K, O, H)=$

$\begin{array}{lllllllllllllll}1 & 2 & 3 & 4 & 5 & 6 & 7 & 8 & 9 & 10 & 11 & 12 & 13 & 14 & 15\end{array}$

I THR LEU LEU SER ALA GLU GLU ASP ALA his VAl thr SER Leu trp GLY LYS VAL ASN VAL GLU LYS VAL GLY GLY GLU ALA LEU GLY ARG

31 LEU LEU VAL VALITYR,PRo, TRP, THR, GLU,ARG, PHE, PHE, GLU, SER,PHE, GLY, ASP =LEU, SER, SER, PRO, SER, ALA, VAL, MET, GLY, ASP, PRO, LYS, YAL,

61 LYS,ALA,HIS, GLY, LYS, LYS, VAL, LEU, SER, ALA, PHE, SER, GLU, GLY=LEU, HIS,HIS, LEU, ASP, ASP, LEU,LYS, GLY, THR, PHE, ALA, ALA, LEU, SER, GLU,

91 LEU,HIS, CYS, VAL, ALA, LEU, HIS, VAL, ASP, PRO, GLU, ASP, PHE, LYS, LEU, $L E U, G L Y, A S P, S E R, L E U, S E R, A S P, V A L, L E U, A L A, A S P, H I S, P H E, G L Y, L Y S)$

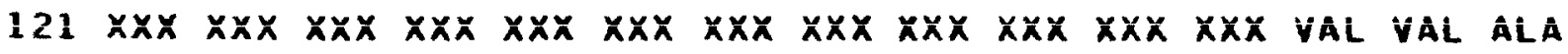
GLY VALIALA,ASP, ALA,LEU, ALA,HIS,LYS, TYR,HIS)**

\section{COMPOSITION}

\begin{tabular}{|c|c|c|c|c|c|c|c|c|c|c|}
\hline 14 & ALA & $\mathbf{A}$ & 0 & GLN & 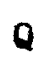 & 19 & LEU & $L$ & 11 & SER \\
\hline 2 & ARG & $\mathbf{R}$ & 9 & GLU & $E$ & 10 & LYS & $K$ & 4 & THR \\
\hline 1 & ASN & $\mathbf{N}$ & 12 & GLY & $G$ & 1 & MET & $M$ & 2 & TRP \\
\hline 11 & ASP & D & 9 & HIS & $H$ & 7 & PHE & $F$ & 2 & TYR \\
\hline 1 & CYS & C & 0 & ILU & I & 4 & PRO & $\mathbf{P}$ & 15 & VAL \\
\hline
\end{tabular}

TOTAL NO. OF $\triangle$ CIDS $=146$

- BUETTNER-JANUSCH, J. AND HILL, R. L. SCIENCE, VOL. 147 , PP. 836-842, FEB. 19, 1965 
Normal adult human hemoglobin (hemoglobin A) contains two pairs of polypeptide chains, termed alpha and beta. Each pair is identical. Some modified beta chains have been given other Greek letters, for example, normal fetal hemoglobin is composed of two alpha chains and two "gamma" chains. Usually, however, al tered hemoglobins are different in only a single amino acid. A number of hemoglobins bearing these altered amino acid sequences in their polypeptide chains have been described. For example, one of the early reports by Ingram (1957) shows the chemical difference between normal human hemoglobin and sickle cell hemoglobin. By comparison of amino acid sequences of tryptic peptide digests of the two hemoglobins, it was established that hemoglobin A (normal) contains a GLU residue in the locus where hemoglobin S (sickle cell) contains VAL. This replacement of two charged GLU residues for two uncharged VAL residues in the hemoglobin tetramer is sufficient to account for the "sickling" phenomenon in the abnormal hemoglobin. Listed below are a number of known amino acid replacements in abnormal human hemoglobins.

\begin{tabular}{|c|c|c|c|c|}
\hline \multirow{3}{*}{$\begin{array}{l}\text { HEMOGLOBIN } \\
\text { NAME } \\
\text { A NORMAL }\end{array}$} & \multicolumn{3}{|c|}{ CHANGES } & \multirow[t]{2}{*}{ REFERENCE } \\
\hline & \multicolumn{3}{|c|}{ CHAIN POS. FROM TO } & \\
\hline & & & & \\
\hline F NORMAL FETAL & BETA & (CALLED & GAMMA) & 1 \\
\hline I & ALPHA & 16 & LYS-ASP & 2 \\
\hline NORFOLK & ALPHA & 57 & GLY-ASP & 3 \\
\hline M BOSTON & ALPHA & 58 & HIS-TYR & 4 \\
\hline M SASKATOON & BETA & 63 & HIS-TYR & 4 \\
\hline M MILWAUKEE & BETA & 67 & VAL-GLU & 4 \\
\hline D PUNJAB & BETA & 121 & GLU-GLN & 5 \\
\hline SAN JOSE & BETA & 7 & GLU-GLY & 6 \\
\hline ZURICH & BETA & 63 & HIS-ARG & 7 \\
\hline c & BETA & 6 & GLU-LYS & 8 \\
\hline 0 ARABIA & BETA & 121 & GLU-LYS & 9 \\
\hline 0 INDONESIA & ALPHA & 116 & GLU-LYS & 9 \\
\hline \multirow[t]{2}{*}{$x$} & ALPHA & 68 & ASN-LYS & \\
\hline & $\begin{array}{l}\text { and } \\
\text { BETA }\end{array}$ & 6 & GLU-LYS & 10 \\
\hline$S$ & BETA & 6 & GLU-VAL & 11 \\
\hline D IBADAN & BETA & 87 & THR-LYS & 12 \\
\hline F TEXAS & GAMMA & 5 or 6 & GLU-LYS & 13 \\
\hline KENWOOD & BETA & 143 & HIS-ASP & 14 \\
\hline G & BETA & 7 & GLU-GLY & 15 \\
\hline
\end{tabular}


1. Rhinesmith, H. W., Schroeder, W. A., and Pauling, L., J. Am. Chem. Soc., Vol. 79, p. 4682, 1957

Rhinesmith, H. W., Schroeder, H. A., and Martin, N., J. Am. Chem. Soc., Vol. 80, p. 3358, 1958

2. Murayama, M., Fed. Proc., Vol. 19, p. 78, 1960

3. Baglioni, C., J. Biol. Chem., Vol. 237, pp. 69-74, 1962

4. Gerald, P. S. and Efron, M. L., Proc. Natl. Acad. Sci. U.S., Vol. 47, pp. 1758-1767, 1958

5. Baglioni, C., Biochim. Biophys. Acta, Vol. 59, pp. 437-440, 1962

6. Hill, R. L. and Schwartz, H. C., Nature, Vol. 184, pp. 641-642, 1959

7. Muller, C. J. and Kingma, S., Biochim. Biophys. Acta, Vol. 50, p. 595, 1961

8. Hunt, J. A. and Ingram, V. M., Nature, Vol. 184, p. 640, 1959

Ingram, V. M., Nature, Vol. 180, pp. 326-328, 1957

9. Baglioni, C. and Lehmann, H., Nature, Vol. 196, pp. 229-232, 1962

10. Baglioni, C. and Ingram, V. M., Nature, Vol. 189, pp. 465-467, 1961

11. Ingram, V. M., Nature, Vo1. 180, pp. 326-328, 1957

12. Watson-Williams, E. J., Nature, Vol. 205, pp. 1273-1276, 1965

13. Schneider, R. G., Science, Vol. 148, pp. 240-242, 1965

14. Beale, D. and Lehmann, H., Nature, Vol. 207, pp. 249-261, 1965

15. Hill, R. L., Swenson, R. T., and Schwartz, H. C., J. Biol. Chem., Vol. 235, pp. 3182-3187, 1960 
MYOGLOBIN - WHALE

123456789012334567896012234567890

IVLSEGEHOLVLHVHAKVEADVAGHG O D I LI

31 RLFKSHPETLEKF DRFKHLKTEAEMKA SE O

61 LKKHGVTVLTALGAILKKKGHHEAELKPLA

91 Q SHATKHKI PIKOLEFISEAIIH VLHSRH P 121 G NF GA DAOGAMNKALELFRKDIAAKOKELG $15100 \mathrm{Q} *$

$\begin{array}{lllllllllllllll}1 & 2 & 3 & 4 & 5 & 6 & 7 & 8 & 9 & 10 & 11 & 12 & 13 & 14 & 15\end{array}$

I VAL LEU SER GLU GLY GLU TRP GLN LEU VAL LEU His VAL TRP aLA LyS VAL GLU ALA ASP VAL ALA GLY HIS GLY GLN ASP ILU LEU ILU

31 ARG LEU PHE LYS SER HIS PRO GLU THR LEU GLU LYS PHE ASP ARG

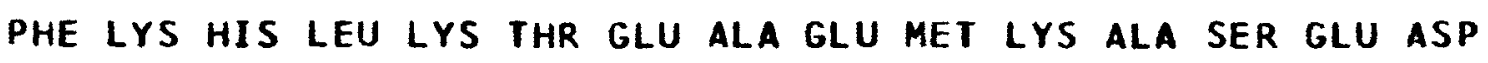
61 LEU LYS LYS HIS GLY VAL THR VAL LEU THR ALA LEU GLY ALA ILU LEU LYS LYS LYS GLY HIS HIS GLU ALA GLU LEU LYS PRO LEU ALA

91 GLN SER HIS ALA THR LYS HIS LYS ILU PRO ILU LYS TYR LEU GLU Phe ILU SER glu ala ILU ILU his VAL LeU His SER arg his pro 121 gly asn phe gly ala asp ala gLn gLy aLA met asn lys ala leu gLU LEU PHE ARG LYS ASP ILU ALA ALA LYS TYR LYS GLU LEU GLY 151 TYR GLN GLY ***

\section{COMPOSITION}

\begin{tabular}{|c|c|c|c|c|c|c|c|c|c|c|}
\hline 17 & ALA & $A$ & 5 & GLN & 0 & 18 & LEU & $\mathbf{L}$ & 6 & SER \\
\hline 4 & ARG & $\mathbf{R}$ & 14 & GLU & $E$ & 19 & LYS & $k$ & 5 & THR \\
\hline 2 & ASN & $\mathbf{N}$ & 11 & GLY & 6 & 2 & MET & $M$ & 2 & TRP \\
\hline 6 & ASP & D & 12 & HIS & $H$ & 6 & PHE & $F$ & 3 & TYR \\
\hline 0 & CYS & $C$ & 9 & ILU & I & 4 & PRO & $\mathbf{P}$ & 8 & VAL \\
\hline
\end{tabular}

- EDMUNDSON, A. B.. NATURE, VOL.205, N0.4974, PP.883-887, FEBRUARY 27,1965 
DiHeme PEPTIDE - ChromatiUm

THE PEPTIDE CONTAINS Iho heme gROUPS. THE FIRST IS COVALENTLY BONDED TO CYSTEINES 5 AND 8. THERE IS ONLY ONE OTHER CYSTEINE AVAILABLE FOR THE OBSERVEd COVALENT BONDING OF THE SECOND HEME.

123456789012345678901234567

I FAGKCSOCHTLVADEGSAKCHTFDEGS/

$\begin{array}{lllllllllllllll}1 & 2 & 3 & 4 & 5 & 6 & 7 & 8 & 9 & 10 & 11 & 12 & 13 & 14 & 15\end{array}$

"/ Phe ala gly lys cys ser gln cys his thr leu val ala asp glu

GLY SER ALA LYS CYS HIS THR PHE ASP GLU GLY SER //

\section{COMPOSITION}

$\begin{array}{lllllllll}3 \text { ALA A } & 1 \text { GLN } & 0 & 1 \text { LEU L } & 3 \text { SER } & S \\ 0 \text { ARG } & R & 2 \text { GLU } & \text { E } & 2 \text { LYS } K & 2 \text { THR } & \text { T } \\ 0 \text { ASN N } & 3 \text { GLY } & 6 & 0 \text { MET } & \text { M } & 0 \text { TRP } & \text { N } \\ 2 \text { ASP } & \text { D } & 2 \text { HIS } & \text { H } & 2 \text { PHE F } & 0 \text { TYR } & 0 \\ 3 \text { CYS } & \text { C } & 0 \text { ILU } & \text { I } & 0 \text { PRO P } & 1 \text { VAL } V\end{array}$

TOTAL ND. OF ACIDS IN FRAGMENT $=27$

- dus,k., Barts'ch, R.g., and Kamen,M.D., J. BIol. Chem., vol.237, NO.10, PP.3083-3093, OCT., 1962 
THE PROTEIN CONTAINS 7 SULPhide AND 7 IRON atoms PER molecule. II DOES NOT CONTAIN HEME.

123456789012345678901234567890

I A OK I A D S C V S C/G A C/ASECPVNAISOG D S I FI $31 V I D A D T C I D C G N C A N V C P V G A P V O E$.

$\begin{array}{lllllllllllllll}1 & 2 & 3 & 4 & 5 & 6 & 7 & 8 & 9 & 10 & 11 & 12 & 13 & 14 & 15\end{array}$

I ALA TYR LYS ILU ALA ASP SER CYS VAL SER CYS/GLY ALA CYS/ALA SER GLU CYS PRO VAL ASN ALA ILU SER GLN GLY ASP SER ILU PHE/

31 VAL ILU ASP ALA ASP THR CYS ILU ASP CYS GLY ASN CYS ALA ASN VAL CYS PRO VAL GLY ALA PRO VAL GLN GLU

\section{COMPOSITION}

\begin{tabular}{|c|c|c|c|c|c|c|c|c|c|c|}
\hline 8 & ALA & $A$ & 2 & GLN & 0 & 0 & LEU & $L$ & 5 & SER \\
\hline jo & ARG & $\mathbf{R}$ & 2 & GLU & $\mathbf{E}$ & 1 & LYS & $k$ & $\mathbf{i}$ & THR \\
\hline 3 & $A S N$ & $\mathbf{N}$ & 4 & GLY & $\mathbf{G}$ & 0 & MET & $M$ & 0 & TRP \\
\hline b & ASP & D & 0 & HIS & $\mathbf{H}$ & 1 & PHE & $F$ & 1 & TYR \\
\hline 8 & CYS & $C$ & 5 & ILU & I & 3 & PRO & $p$ & 6 & VAL \\
\hline
\end{tabular}

- TANAKA, M., NAKASHIMA, T., BENSON,A., MOMER,H.F , ,AND YASUNDBU,K.T., BIOCHEM. BIOPHYS. RES. COMMUN., VOL. 16, NO.5, PP. $422-427,1964$ 
AZURIN - PSEUDOMONAS FLUORESCENS

THE BLUE PROTEIN CONTAINS DNE COPPER ATOM PER MOLECULE.

123456786901223456778960123456678990

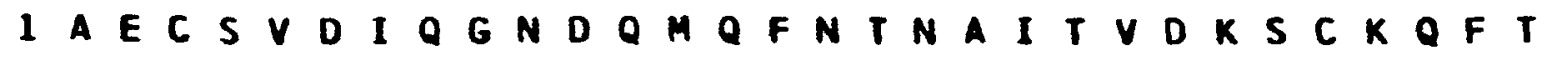

31 V NLSH PGNLPKN V A GHNHVLSTAADM OGVV

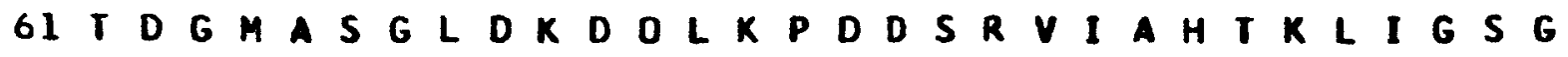

91 E K D S T T D V S KLKEGEODAFF C TFP GHSAL

$121 M K G T L T L K *$

$\begin{array}{lllllllllllllll}1 & 2 & 3 & 4 & 5 & 6 & 7 & 8 & 9 & 10 & 11 & 12 & 13 & 14 & 15\end{array}$

1 ala glu Cys SER VAl ASP ILU GLN GLY ASN ASP GLN MEt GLN PHE ASN THR ASN ALA ILU THR VAL ASP LYS SER CYS LYS GLN PHE THR

31 VAL ASN LEU SER HIS PRO GLY ASN LEU PRO LYS ASN VAL MET GLY hIS ASN tRP VAL LEU SER THR ALA ALA ASP MET GLN GLY VAL VAL

61 THR ASP GLY MET ALA SER GLY LEU ASP LYS ASP TYR LEU LYS PRO ASP ASP SER ARG VAL ILU ALA HIS THR LYS LEU ILU GLY SER GLY

91 GLU LYS ASP SER VAL THR PHE ASP VAL SER LYS LEU LYS GLU GLY glu gln tyr met phe phe cys thr phe pro gly his ser ála leu

121 MET LYS GLY THR LEU THR LEU LYS ***

COMPOSITION

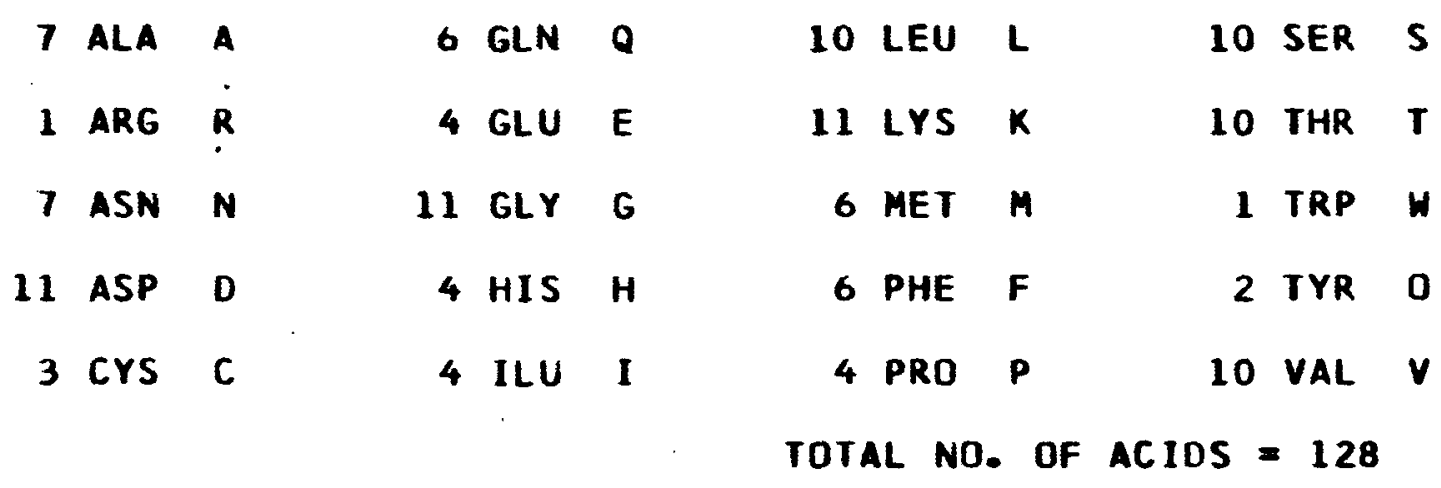

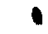

- AMBLER,R.P.,AND BROWN,L.H., J. MOL. BIOL., VOL.9, NO.3, PP. 825-828, SEPT. 1964 


\section{RI BONUCLEASE - BOVINE}

DISULPHIDE BONDS ARE FORMEO BETHEEN CYSTEINES AT POSITIONS 26 AND 84, 40 AND 95, 58 AND 110, AND 65 AND 72.

$\begin{array}{llllllllllllllllllllllllllllll} & 2 & 3 & 4 & 5 & 6 & 7 & 8 & 9 & 0 & 1 & 2 & 3 & 4 & 5 & 6 & 7 & 8 & 9 & 0 & 1 & 2 & 3 & 4 & 5 & 6 & 7 & 8 & 9 & 0\end{array}$

I K E T A A A K FER Q H M O S S T S A A S S S N O C N O M M 31 K S R N L T K D R C K P V N T F V HE SLA D V O A V C S O

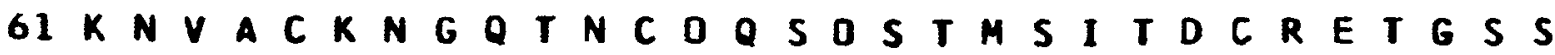
91 KO P N CAOKTT QANKHIIVACEGNPOVPVHF 121 D A S V *

$\begin{array}{lllllllllllllll}1 & 2 & 3 & 4 & 5 & 6 & 7 & 8 & 9 & 10 & 11 & 12 & 13 & 14 & 15\end{array}$

1 Lys glu thr ala ala ala lys phe gLU ARg gLN his met asp ser SER THR SER ALA ALA SER SER SER ASN TYR CYS ASN GLN MET MET

31 LYS SER ARG ASN LEU THR LYS ASP ARG CYS LYS PRO VAL ASN THR

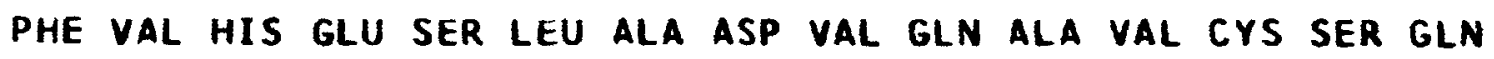
61 LYS ASN VAL ALA CYS LYS ASN GLY GLN THR ASN CYS TYR GLN SER TYR SER THR MET SER ILU THR ASP CYS ARG GLU THR GLY SER SER

91 LYS TYR PRO ASN CYS ALA TYR LYS THR THR GLN ALA ASN LYS HIS ILU ILU VAL ALA CYS GLU GLY ASN PRO TYR VAL PRD VAL HIS PHE 121 ASP ALA SER VAL **:

COMPOSITION

\begin{tabular}{|c|c|c|c|c|c|c|c|c|c|c|}
\hline 12 & ALA & $A$ & 7 & GLN & 0 & 2 & LEU & $\mathbf{L}$ & 15 & SER \\
\hline 4 & ARG & $\mathbf{R}$ & 5 & GLU & $E$ & 10 & LYS & $\mathbf{K}$ & 10 & THR \\
\hline 10 & ASN & $\mathbf{N}$ & 3 & GLY & $\mathbf{G}$ & 4 & MET & $M$ & 0 & TRP \\
\hline 5 & ASP & D & 4 & HIS & $H$ & 3 & PHE & $F$ & 6 & TYR \\
\hline 8 & CYS & C & 3 & ILU & I & 4 & PRO & $P$ & 9 & VAL \\
\hline
\end{tabular}

* SMYTH, D.G., STEIN,H.H. AND MODRE, S., J. BIOL. CHEM., VOL.238, NO.1, PP.227-234, JAN., 1963 
DISULPHIDE BONOS ARE FORMED BETHEEN CYSTEINES AT POSITIONS 5-55, 14-38, AND 30-51.

123456789012345678901234567890

I RPDFCLEPPOTGPCKARIIROFONAKAGLC 31 Q TFVOGGCRAKRNNFKSAEDCMRTCGGA*

$\begin{array}{lllllllllllllll}1 & 2 & 3 & 4 & 5 & 6 & 7 & 8 & 9 & 10 & 11 & 12 & 13 & 14 & 15\end{array}$

1 ARG PRO ASP PHE CYS LEU GLU PRO PRO TYR THR GLY PRO CYS LYS

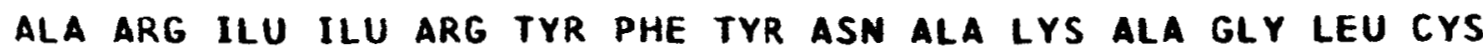

31 GLN THR PHE VAL TYR GLY GLY CYS ARg ALA LYS ARG ASN ASN PHE LYS SER ALA GLU ASP CYS MET ARG tHR CYS GLY GLY ALA **

CDMPOSITION

$\begin{array}{lllllllll}6 \text { ALA } & \text { A } & 1 \text { GLN } & 0 & 2 \text { LEU L } & 1 \text { SER } & S \\ 6 \text { ARG } & \text { R } & 2 \text { GLU } & \text { E } & 4 \text { LYS } & \text { K } & 3 \text { THR } & T \\ 3 \text { ASN N } & 6 \text { GLY } & G & 1 \text { MET } M & 0 \text { TRP } & W \\ 2 \text { ASP } & \text { D } & 0 \text { HIS } & H & 4 \text { PHE F } & 4 \text { TYR } & 0 \\ 6 \text { CYS } & \text { C } & 2 \text { ILU } & \text { I } & 4 \text { PRO P } & 1 \text { VAL } V \\ & - & & & \text { TOTAL NO. OF ACIOS }=58\end{array}$

- KASSEll, B., RADICEVIC, M., ANSFIELd, M. J., AND LASKOWSKi, M., BIOCHEM. BIOPHYS. RES. COMMUN., VOL. 18, NO,2, PP.255-258, 1965

DLOUHA, V., POSPISILOVA, D., MELOUN, B. AND SORM, F., COLLECTION CZECH. CHEM. COMMUN., VOL. 30, PP.1311-1325, 1965

THE SEQUENCE REPORTED HERE DIFFERS FROM THE ABDVE IN HAVING THE ILU (I) DELETED AT POSITION 19. 
TI BOPA 5.001-2

CHAUVET, J., NOUVEL, G., AND ACHER, R., BIOCHIM. BIOPHYS. ACTA, VOL. 92, PP. 200-201, 1964

THE SEQUENCE REPORTED HERE DIFFERS FROM THE ABOVE IN THE FOLLOWING RESPECTS.

THE ARG (R) FROM POSITION 42 HAS BEEN REMOVED AND INSERTED

BETWEEN POSITIONS 20 AND 21. THE GLN (Q) AT POSITION 31 HAS BEEN DELETED AND A GLU (E) ADDED BeTHEEN POSITIONS 32 AND 33.

KASSELL, B., AND LASKOWSKI, M., BIOCHEM. BIOPHYS. RES. CUMMUN. VOL 20, NO.4, PP.463-468, 1965 
TOBACCO MOSAIC VIRUS

ACETYL - AT AMINO END

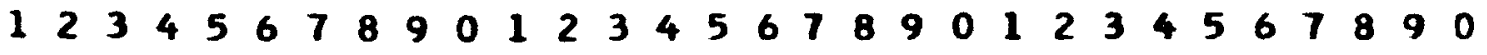

I S O S I T T P S Q F V FL S S A HA D P I EL I N L C T N A

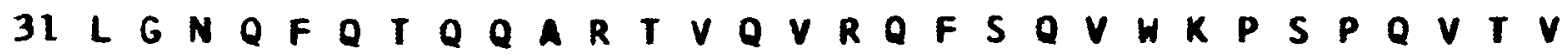
61RFP DSDFKVORONAVLDPLVTALLGAFATR 91 N R I I Q V Q D O A N P T T A Q T L D A T R R V D D A T VA 121 I R S A D I N L I V E L I R G T G S O N R S S F E S S S G L 151 V TS GPAT

$\begin{array}{lllllllllllllll}1 & 2 & 3 & 4 & 5 & 6 & 7 & 8 & 9 & 10 & 11 & 12 & 13 & 14 & 15\end{array}$

I SER TYR SER ILU THR THR PRO SER GLN PHE VAL PHE LEU SER SER ALA trp alA ASP PRO ILU GLU LEU ILU ASN LEU CYS THR ASN ALA

31 LEU GLY ASN GLN PHE GLN THR GLN GLN ALA ARG THR VAL GLN VAL ARG GLN PHE SER GLN VAL TRP LYS PRO SER PRO GLN VAL THR VAL

61 ARG PHE PRO ASP SER ASP PHE LYS VAL TYR ARG TYR ASN ALA VAL

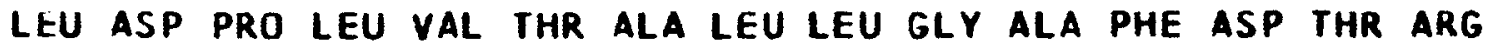

91 ASN ARG ILU ILU GLN VAL GLN ASP GLN ALA ASN PRO THR thr aLA GLN THR LEU ASP ALA THR ARG ARG VAL ASP ASP ALA THR VAL ALA 121 ILU ARg SER ALA ASP ILU ASN LEU ILU YAL GLU LEU ILU ARG GLY THR GLY SER TYR ASN ARG SER SER PHE GLU SER SER SER GLY LEU 151 VAL TRP THR SER GLY PRO ALA THR **

\section{COMPOSITION}

\begin{tabular}{|c|c|c|c|c|c|c|c|c|c|c|}
\hline 14 & ALA & A & 13 & GLN & $\mathbf{a}$ & 12 & LEU & $L$ & 16 & SER \\
\hline 11 & ARG & $\mathbf{R}$ & 3 & GLU & $\mathbf{E}$ & 2 & LYS & $k$ & 16 & THR \\
\hline 8 & ASN & $N$ & 6 & GLY & G & 0 & MET & $\boldsymbol{M}$ & 3 & TRP \\
\hline 10 & ASP & D & 0 & HIS & $H$ & 8 & PHE & $F$ & 4 & TYR \\
\hline 1 & CYS & $C$ & 9 & ILU & $\mathbf{I}$ & 8 & PRD & $\mathbf{P}$ & 14 & VAL \\
\hline
\end{tabular}

TOTAL NO. OF ACIDS $=158$ 


\section{TM TM $6.001-2$}

- ANDERER, F.A., Z. NATURFORSCH., VOL. 17, PP.526-543, 1962 STRUCTURE REVISIONS AND CONFIRMATIONS.

ANDERER, F.A., UHLIG, H., HEBER, E., AND SCHRAMM, G., NATURE, VOL. 186, NO.4729, PP.922-925, JUNE 18, 1960

FUNATSU, G., TSUGITA, A., AND FRAENKEL-CONRAT, H., ARCH. BIOCHEH. BIOPHYS., VOL. 105, NO.1, PP.25-41, APR. 1964 
TOBACCO MOSAIC VIRUS STRAIN DAHLMENSE

ACETYL- AT AMINO END

123456789012345678901234567890

IS OS I T SPS QFVFLLSSVHADPIELLNVCISS

31 LGNQFQTQOARTTOVQQFSEVWKPFPQSTV 61 RFPGDVOKVORONAVLDPLITALLGTFDTR 91 NR I I E VEN Q Q S P T TAETLDATRR VD DATIVA 121 I RSANINLVNELVRGTGLONONTFESMSGL 151 V W S A PAS -

$\begin{array}{lllllllllllllll}1 & 2 & 3 & 4 & 5 & 6 & 7 & 8 & 9 & 10 & 11 & 12 & 13 & 14 & 15\end{array}$

1 SER tyr ser ilu thr SER PRo SER GLN PHE VAL PHe LeU SER SER VAL TRP ALA ASP PRO ILU GLU LEU LEU ASN VAL CYS THR SER SER 31 LEU GLY ASN GLN PHE GLN THR GLN GLN ALA ARG THR THR GLN VAL GLN GLN PHE SER GLU VAL tRP LYS PRO PHE PRO GLN SER thr VAL 61 ARG PHE PRO GLY ASP VAL TYR LYS VAL TYR ARG TYR ASN ALA VAL LEU ASP PRO LEU ILU THR ALA LEU LEU GLY THR PHE ASP THR ARG gi ASN ARG ILU ILU GLU VAL GLU ASN GLN GLN SER PRO THR THR ALA GLU THR LEU ASP ALA thr ARg ARg VAL ASP ASP ALA THR VAL ALA 121 ILU ARg SER ALA ASN ILU ASN LEU VAL ASN GLU LEU VAL ARg GLY thr GLY LEU tYr ASN GLN ASN THR PHE GLU SER MET SER GLY LEU 151 VAL TRP THR SER ALA PRO ALA SER ***

COMPOSITION

$\begin{array}{rlrllllll}11 \text { ALA A } & 12 \text { GLN } & \text { Q } & 13 \text { LEU L } & 16 \text { SER } & \text { S } \\ 9 \text { ARG } & \text { R } & 7 \text { GLU } & \text { E } & 2 \text { LYS } & \text { K } & 17 \text { THR } & \text { T } \\ 10 \text { ASN N } & 6 \text { GLY } & \text { G } & 1 \text { MET } & \text { M } & 3 \text { TRP } & \text { H } \\ 7 \text { ASP } & \text { D } & 0 \text { HIS } & \text { H } & 8 \text { PHE F } & 5 \text { TYR } & \text { F } \\ 1 \text { CYS } \text { C } & 7 \text { ILU } & \text { I } & 8 \text { PRO P } & 15 \text { VAL } V\end{array}$

TOTAL NO. OF ACIDS $=158$ 
TM TMD 6.002-2

- WITTMANN-LIEBOLD,B. AND WITTMANN, H. G., Z. VERERBUNGS., VUL. 94, PP. 427-435, 1963 
CHYMOTRYPSINOGEN-A - BOVINE

12345678901223456789901234567890

I C G V P I Q PVLS GLSRIVG DEEAVPG SWPHO 31 V L U DKT GFHF C G G SLINENWVVIAAHCGV 61 T S DVVVAGEF D Q G S S SEKI Q KLKIAK VFK 91 N S K O N S L T I N N N I T L L K L S TA A S F S O T V S A 121 V C L P S A S D D FA A G T T C V T T G H G L T R O T NA N 151 T P D R L Q A S L PLLS N T N C K K O W G TKK I K D A A 181 I C A G A S G S S C M G D S G G PLVCKKNGA HTLV 211 G IVSWGSSTCSTTST P G V OAR V TA L V N W V Q Q 241 T L A A

$\begin{array}{lllllllllllllll}1 & 2 & 3 & 4 & 5 & 6 & 7 & 8 & 9 & 10 & 11 & 12 & 13 & 14 & 15\end{array}$

I CyS gLY VAL PRO ALA ILU GLN PRO VAL LEU SER GLY LEU SER ARG ILU VAL GLY ASP GLU GLU ALA VAL PRO GLY SER TRP PRO TRP GLN

31 VAL SER LEU GLN ASP LYS THR gLY PHE HIS PHE CYS GLY GLY SER LEU ILU ASN GLU ASN TRP VAL VAL THR ALA ALA HIS CYS GLY VAL 61 THR THR SER ASP VAL VAL VAL ALA GLY GLU PHE ASP GLN GLY SER SER SER GLU LYS ILU GLN LYS LEU LYS ILU ALA LyS VAL PHE LyS

91 ASN SER LYS TYR ASN SER LEU THR ILU ASN ASN ASN ILU THR LEU LEU LyS LEU SER thr aLA ALA SER PHE SER GLN thr VAL SER ALA 121 VAL CYS LEU PRO SER ALA SER ASP ASP PHE ALA ALA GLY THR THR CYS VAL THR THR GLY TRP GLY LEU THR ARG TYR THR ASN ALA ASN 151 THR PRO ASP ARG LEU GLN GLN ALA SER LEU PRO LEU LEU SER ASN THR ASN CYS LYS LYS TYR TRP GLY THR LYS ILU LYS ASP ALA MET 181 ILU CYS ALA GLY ALA SER GLY VAL SER SER CYS MET GLY ASP SER GLY GLY PRD LEU VAL CYS LYS LYS ASN GLY ALA TRP THR LEU VAL 211 GLY ILU YAL SER TRP GLY SER SER THR CYS SER THR SER THR PRD GLY VAL TYR ALA ARG VAL IHR ALA LEU VAL ASN TRP VAL GLN GLN 
COMPOSITION

\begin{tabular}{|c|c|c|c|c|c|c|c|c|c|c|}
\hline 22 & $A L A$ & $A$ & 10 & GLN & $Q$ & 19 & LEU & $\mathbf{L}$ & 28 & SER \\
\hline 4 & ARG & $R$ & 5 & GLU & $E$ & 14 & LYS & $\mathbf{K}$ & 23 & THR \\
\hline 14 & ASN & $\mathbf{N}$ & 23 & GLY & $\mathbf{G}$ & 2 & MET & $\mathbf{M}$ & 8 & TRP \\
\hline 9 & ASP & D & 2 & HIS & H & 6 & PHE & $F$ & 4 & TYR \\
\hline 10 & CYS & C & 10 & $\mathbf{I L U}$ & I & 9 & PRO & $\mathbf{P}$ & 23 & VAL \\
\hline
\end{tabular}

- HARILEY, B.S.. BRDWN, J.R., KAUFFMAN, D.L., AND SMILLIE,L.R., NATURE, VOL.207, NO.5002, PP.1157-1159, SEPT.11, 1965

THIS SEQUENCE HAS BEEN CORRECTED BY DELETING SER (S)

WHICH WAS AT POSITIUN 215.

BROHN, J.R., AND HARTLEY, B. S., BIDCHEM J.. VOL. 89, 59P, 1963

THE ACTIVE SITE SERINE IS AT POSITION 195

KEIL, B., PRUSIK, Z., AND SORM, F., BIOCHIM. BIUPHYS. ACTA. VOL. 78, P. 559-561, 1963

DISULPHIDE BRIDGES LINK POSITIONS 1-122, 42-58, 136-201, 168-182 AND 191-220.

KOSTKA, V., MELOUN, B., AND SORM,F., COLLECTION CZECH.

CHEM. COMMUN.. VOL. 28, PP.2779-2805, 1963 .

HARTLEY,B.S', NATURE, VOL. 201, NO. 4962, PP.1284-1287, MARCH 28,1964 


\section{TRYPSINOGEN}

123456789012345678901234567890

IVDDDDKIVGG OTCGANTVPOOVSLNSGOHF 31 C G GSLINSOWVVSAAHCOKSGIOVRLGEDN 61 I NVVEG DEOFISASKSIVHPSON(P,L,T,NIN N D 91 I MLIKLKSAASLNSRVASISLPTSGASAGT 121 OCLIS GWGNTKSSGTSOPDVLKCLKAPILS 151 DSSCKSAOPG OITSNMFCAGOLEGGKNSCO 181 G DS G GPVVCSGKL Q GIVSWGSGCAQKNKPG $211 V O T K V C N O V S$ I K O T I A S N.

$\begin{array}{lllllllllllllll}1 & 2 & 3 & 4 & 5 & 6 & 7 & 8 & 9 & 10 & 11 & 12 & 13 & 14 & 15\end{array}$

1 VAL ASP ASP ASP ASP LYS ILU VAL GLY GLY TYR THR CYS GLY ALA ASN THR VAL PRO TYR GLN VAL SER LEU ASN SER GLY TYR HIS PHE

31 CYS GLY GLY SER LEU ILU ASN SER GLN TRP VAL VAL SER ALA ALA HIS CYS TYR LYS SER GLY ILU GLN VAL ARG LEU GLY GLU ASP ASN

61 ILU ASN VAL VAL GLU GLY ASP GLU GLN PHE ILU SER ALA SER LYS SER ILU VAL HIS PRO SER TYR ASNIPRO,LEU,THR, ASNIASN ASN ASP

91 ILU met LeU ILU LyS LEU LyS SER ALA ALA SER Leu ASN SER ARg VAL ALA SER ILU SER LEU PRO THR SER CYS ALA SER ALA GLY THR 121 GLN CYS LEU ILU SER GLY TRP GLY ASN THR LYS SER SER GLY THR SER tYr PRo ASP VAL LEU LYS CYS LEU LYS ALA PRO ILU LEU SER 151 ASP SER SER CYS LYS SER ALA TYR PRO GLY GLN ILU THR SER ASN MET PHE CYS ALA GLY TYR LEU GLU GLY GLY LYS ASN SER CYS GLN 181 GLY ASP SER GLY GLY PRO VAL VAL CYS SER GLY LYS LEU GLN GLY ILU VAL SER TRP GLY SER GLY CYS ALA GLN LYS ASN LYS PRO GLY 211 VAL TYR THR LYS VAL CYS ASN TYR VAL SER TRP ILU LYS GLN THR ILU ALA SER ASN $*$ 
TR BOTR 7.002-2

COMPOSITION

\begin{tabular}{|c|c|c|c|c|c|c|c|c|c|c|}
\hline 14 & ALA & A & 10 & GLN & $Q$ & 14 & LEU & $L$ & 33 & SER \\
\hline 2 & ARG & $\mathbf{R}$ & 4 & GLU & $\mathbf{E}$ & 15 & LYS & $k$ & 10 & THR \\
\hline 6 & ASN & $\mathbf{N}$ & 25 & GLY & $\mathbf{G}$ & 2 & MET & $M$ & 4 & TRP \\
\hline 10 & ASP & D & 3 & HIS & $H$ & 3 & PHE & $F$ & 10 & TYR \\
\hline ? & CYS & C & 15 & ILU & I & 9 & PRO & $\mathbf{P}$ & 18 & VAL \\
\hline
\end{tabular}

TOTAL NO. OF ACIDS $=229$

- HALSH, K.. AND NEURATH, H., PRDC. NATL. ACAD. SCI. U.S., VOL. 52, NO.4, PP.884-889, 1964

KAUFFMAN, D. L., J. MOL. BIOL., VOL.12, PP.929-932, 1965

DISULPHIDE BRIDGES HERE FOUND BETHEEN LINKS 13-143, 31-47 $115-216,122-189,154-168$, AND 179-203.

THE ACTIVE SER INE IS AT LINK 183. 
PAPAIN

123456789012345678901234567890 1/IPEOVDWROKGAVTPVKNOGSCGSCW/AF/III 31 R N T P O OEG VOR OCRSREKG P OAAKT D G V R O 61VOPONQGALLOSIANQPSVVLOAAGKDFAL 91 ORG GIFVGPCGNKVDHAVAAVGONPGOILLI

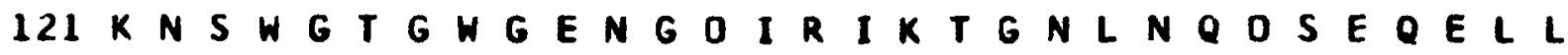
151 D C D R R S O G C O P G D G WIS A L/VA Q O G I H O R G T G $181 N$ S O G V GLOTSSFOPVKN

$\begin{array}{lllllllllllllll}1 & 2 & 3 & 4 & 5 & 6 & 7 & 8 & 9 & 10 & 11 & 12 & 13 & 14 & 15\end{array}$ 1/ILU PRO GLU TYR VAL ASP TRP ARG GLN LYS GLY ALA VAL THR PRO VAL LYS ASN GLN GLY SER CYS GLY SER CYS TRP/ALA PHE/ILU ILU/ 31 ARG ASN THR PRO TYR TYR GLU GLY VAL GLN ARG TYR CYS ARG SER ARG GLU LYS GLY PRO TYR ALA ALA LYS THR ASP GLY VAL ARG GLN

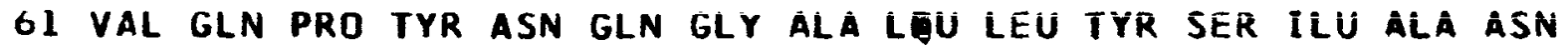
gLN pro ser val val Leu glN ala ala gly lys asp phe glN Leu

91 TYR ARG GLY GLY ILU PHE VAL GLY PRO CYS GLY ASN LYS VAL ASP his ala val ala ala val gly tyr asn pro gly tyr ILU LeU ILU 121 LYS ASN SER TRP GLY THR GLY TRP GLY GLU ASN GLY TYR ILU ARG ILU LYS THR GLY ASN LEU ASN GLN TYR SER GLU GLN GLU LEU LEU 151 ASP CYS ASP ARG ARG SER TYR GLY CYS TYR PRO GLY ASP GLY TRPJ SER ALA LEU/VAL ALA GLN TYR GLY ILU HIS TYR ARG GLY THR GLY 181 ASN SER TYR GLY VAL CYS GLY LEU TYR THR SER SER PHE TYR PRO VAL LYS ASN *** 
PA PA $7.101-2$

\section{COMPOSITION}

\begin{tabular}{|c|c|c|c|c|c|c|c|c|c|c|}
\hline 13 & ALA & $A$ & 12 & GLN & $Q$ & 10 & LEU & $L$ & 12 & SER \\
\hline 11 & ARG & $\mathbf{R}$ & 6 & GLU & $E$ & 9 & LYS & $k$ & 7 & THR \\
\hline 12 & $A S N$ & $\mathbf{N}$ & 27 & GLY & G & 0 & MET & $M$ & 5 & TRP \\
\hline 7 & ASP & D & 2 & HIS & $H$ & 4 & PHE & $\mathbf{F}$ & 19 & TYR \\
\hline 7 & Crs & $C$ & 10 & ILU & $\mathbf{I}$ & 10 & PRO & $\mathbf{P}$ & 15 & VAL \\
\hline
\end{tabular}

- LIGHT, A., FRATER, R. KIMMEL, J., AND SMITH, E.L., PRDC. NATL. ACAD. SCI. U.S., VOL.52, N0.5, PP.1276-1283, NDV. 1964 DISULPHIDE BRIDGES ARE FORMED BETWEEN CYSTEINES AT POSITIONS 43 AND 152, 100 AND 186, AND 22 AND 159.

THE ACTIVE SULFHYORYL GROUP IS AT POSITION 25. 


\section{LYSOZYME - CHICKEN}

LYSOZYME HAS A BETA $(1-4)$ GLUCOSAMINIDASE ACTIVITY HITH THE ABILITY TO HYDROLYSE A MUCOPOL YSACCHARIDE COMPONENT OF SOME BACTER IAL CELL WALLS RELEASING N-ACETYL AMINO SUGARS DERIVED FROM GLUCOSAMINE AND MURAMIC ACID.

123456789012345678901234567890

IKVFGRCELAAAMKRHGLDNORGOSLGNWVC 31 A A KFESNFNTAATNRNTOGGSTDOOGILLOINS 61RHWCNDGRTPGSRNLCNIPCSALLSSDITA 91 S V N CAKKIVSDGDGMNAWVAWRNRCKGT DV $1210 A$ IRGCRL*

$$
\begin{array}{lllllllllllllll}
1 & 2 & 3 & 4 & 5 & 6 & 7 & 8 & 9 & 10 & 11 & 12 & 13 & 14 & 15
\end{array}
$$

1 Lys val phe gly arg cys glu leu ala ala ala met lys arg his GLY LEU ASP ASN TYR ARG GLY TYR SER LEU GLY ASN TRP VAL CYS

31 ala ala lys phe gLU Ser asn phe asn thr gLN ala thr asn arg ASN THR ASP GLY SER THR ASP TYR GLY ILU LEU GLN ILU ASN SER

61 ARG TRP TRP CYS ASN ASP GLY ARG THR PRO GLY SER ARG ASN LEU CYs aśn ilu pro crs ser ala leu leu ser ser asp ilu thr ala 91 SER VAL ASN CYS ALA LYS LYS ILU VAL SER ASP GLY ASP GLY MET ASN ALA TRP VAL ALA TRP ARG ASN ARG CYS LYS GLY THR ASP VAL 121 GLN ALA TRP ILU ARG GLY CYS ARG LEU ***

\section{COMPOSITION}

$\begin{array}{lllllllll}12 & \text { ALA } & \text { A } & 3 \text { GLN } & Q & 8 \text { LEU L } & 10 \text { SER } & \text { S } \\ 11 \text { ARG } & \text { R } & 2 \text { GLU } & \text { E } & 6 \text { LYS } & \text { K } & 7 \text { THR } & \text { T } \\ 13 \text { ASN N } & 12 \text { GLY } & \text { G } & 2 \text { MET } & \text { H } & 6 \text { TRP } & \text { W } \\ 8 \text { ASP } & \text { D } & 1 \text { HIS } & \text { H } & 3 \text { PHE F } & 3 \text { TYR } & 0 \\ 8 \text { CYS } & \text { C } & 6 \text { ILU } & \text { I } & 2 \text { PRO P } & 6 \text { VAL } V\end{array}$

TOTAL NO. OF ACIDS $=129$ 
- CANFIELD, R., J. BIOL. CHEM., VOL.238, ND.8, PP.2698-2707. AUG. 1963

CANFIELD, R., LIU,A.K., J. BIOL. CHEM., VOL.240, NO.5, PP. 1997-2002, MAY 1965

ABOVE SEQUENCE CONFIRMED IN THIS HORK.

DISULPHIDE BONDS ARE FOUND BETHEEN 6 AND 127, 30 AND 115, 64 AND 80, AND 76 AND 94.

JOLLES, J., JAUREGUI-ADELL, J., BERNIER, I. , AND JOLLES, P., BIDCHIM. BIOPHYS. ACTA, VOL.78, PP.668-689, 1963

THIS SEQUENCE DIFFERS FROM THE ABOVE AS FOLLOHS, 40-GLN, 41-ALA, 42-THR, 43-THR, 46-ASP, 58-ASN, 59-ILU, 92-ASN, AND 93-VAL.

BLAKE, C.C.F., KOENING, D.F., MAIR, G.A., NORTH, A.C.T., PHILLIPS, D.C., AND SARMA, V.R., NATURE, ND. 4986, PP. 757-761, MAY 22, 1965

A 2 ANGSTROM RESOLUTION FOURIER SYNTHESIS HAS BEEN PERFORMED BY X-RAY CRYSTALLOGRAPHIC METHODS. THE LOCATION OF THE FDUR DISULPHIDE BRIDGES HAS BEEN CONFIRMED. THE BINDING SITE OF THE INHIBITOR N-ACETYL-GLUCOSAMINE AND ITS DIMER HAS BEEN FOUND TO BE VERY EXTENSIVE INVOLVING RESIDUES AT POSITIONS $44,46,47,48,50,52,57,59,61-63,72,73,97,99-101$, $103,107-110,113$, AND 114. 


\section{GLUCAGON - BOVINE}

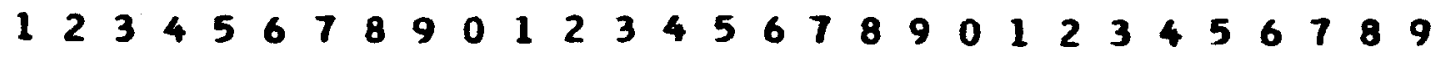

I HS Q G TFT SDOSKOLDSRRAODFVOHLANTH

$\begin{array}{lllllllllllllll}1 & 2 & 3 & 4 & 5 & 6 & 7 & 8 & 9 & 10 & 11 & 12 & 13 & 14 & 15\end{array}$

1 HIS SER GLN GLY THR PHE THR SER ASP TYR SER LYS TYR LEU ASP SER ARG ARG ALA GLN ASP PHE VAL GLN TRP LEU MET ASN THR **

\section{COMPOSITION}

\begin{tabular}{|c|c|c|c|c|c|c|c|c|c|c|}
\hline 1 & ALA & $A$ & 3 & GLN & $a$ & 2 & LEU & $\mathbf{L}$ & 4 & SER \\
\hline 2 & ARG & $\mathbf{R}$ & 0 & GLU & $\mathbf{E}$ & 1 & LYS & $k$ & 3 & THR \\
\hline 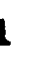 & $A S N$ & $\mathbf{N}$ & 1 & GLY & $\mathbf{G}$ & 1 & MET & $M$ & 1 & TRP \\
\hline 3 & ASP & $\mathbf{D}$ & 1 & HIS & H & 2 & PHE & $F$ & 2 & TYR \\
\hline & CYS & $c$ & 0 & ILU & I & 0 & PRD & $P$ & 1 & VAL \\
\hline
\end{tabular}

- BROMER, W.H., SINN, L.G., AND BEHRENS, 0.K., J. AM. CHEM. SOC., VOL. 79, PP. 2807-2810, JUNE 5, 1957 
THE C TERMINAL gLYCINE IS PRESENT AS THE AMIDE.

THE TWO CYSTEINES ARE LINKED BY A DISULPHIDE BOND.

123456789

ICOFONCPRG

$\begin{array}{lllllllll}1 & 2 & 3 & 4 & 5 & 6 & 7 & 8 & 9\end{array}$

1 CYS TYR PHE GLN ASN CYS PRO ARG GLY \#.**

COMPOSITION

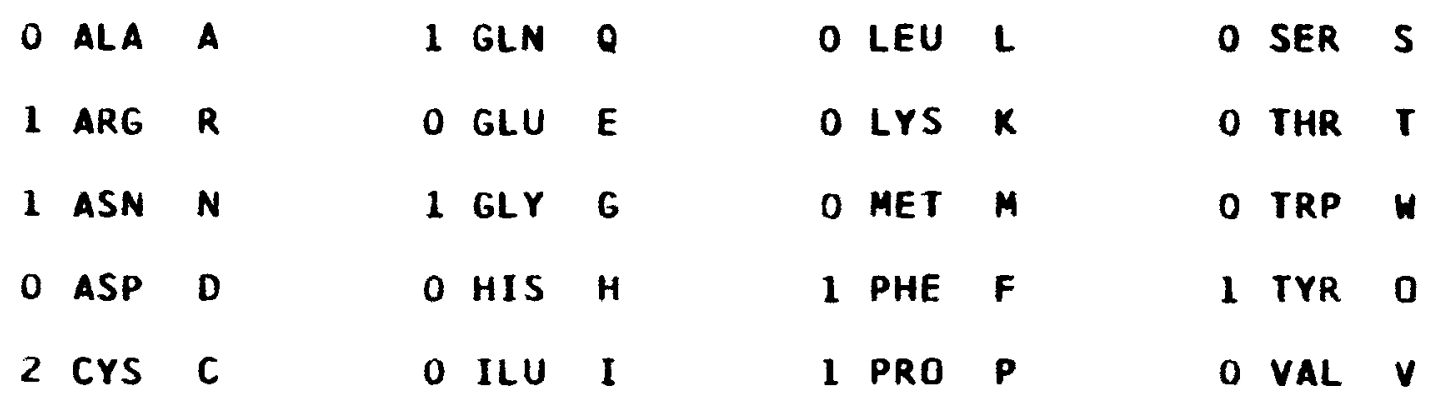

TOTAL NO. OF ACIDS $=9$

- du Vigneaud, V., Lahler, H. C., and popenoe, e. A., J. AM. CHeM. SOC., VOL.75, PP.4880-4881, OCT. 5, 1953

ACHER, R., AND CHAUVET, J., BIOCHIM. BIOPHYS. ACTA, VOL. 12, PP.487-488, 1953

THIS WORK CONFIRMED THE SEQUENCE ABOVE, HOWEVER GLU (E) AND ASP (D) WERE NOT DISTINGUISHED FROM GLN (Q) AND ASN (N). 
THE C TERMINAL GLYCINE IS PRESENT AS THE AMIDE.

THE TWO CYSTEINES ARE LINKED BY A DISULPHIDE BOND.

123456789

1 C.O.F.Q.N.C.P.K.G.*

$\begin{array}{lllllllll}1 & 2 & 3 & 4 & 5 & 6 & 7 & 8 & 9\end{array}$

1 CYS.TYR.PHE.GLN.ASN.CYS.PRD.LYS.GLY **

COMPOSITION
- ala a
1 GLN 0
o LEU L
- SER S
o ARG R
- GLU E
1 LYS $K$
O THR T
1 ASN N
I GLY G
O MET $M$
O TRP H
O ASP D
O HIS H
1 PHE F
1 TYR 0
2 CYS C
o ILU I
1 PRO P
o val v
TOTAL NO. OF ACIDS $=9$

* POPENOE, E. A., LAWLER, H. C.. AND DU VIGNeAUd, Y.,

J. AH. CHEM. SOC., VOL.74, P.3713, JULY 20, 1952 


\section{OXYTOCIN - BOVINE}

THE C TERMinal gLYCINe is pResent as the amide. THE THO CYSTEINES ARE LINKED BY A DISULPHIDE BOND. OXYTOCIN IS THE PRINCIPAL UTERINE CONTRACTING AND MILK EJECIING HORMONE OF THE POSTERIOR PITUITARY.

123456789

ICOIONCPLG.

$\begin{array}{lllllllll}1 & 2 & 3 & 4 & 5 & 6 & 7 & 8 & 9\end{array}$

1 CYS TYR ILU GLN ASN CYS PRO LEU GLY *..*

\section{COMPOSITION}

o ala a

O ARG R

1 ASN N

- ASP D

2 crs C
1 GLN Q

O GLU E

1 GLY G

0 HIS H

1 ILU I
1 LEU L

0 LYS K

O MET M

O PHE F

1 PRO P

TOTAL NO. OF ACIOS $=9$

- DU VIGNEAUd, V., RESSleR, C., TRIPPETT, S., J. BIOL. CHEM., VOL.205, PP.949-957, 1953

TUPPY, H. AND MICHL, H., MONATSH. CHEM., VOL.84, PP.1011-1020, 1953 
HYPERTENSIN - BOVINE

1234567890

I D R V V H P F H L

$\begin{array}{llllllllll}1 & 2 & 3 & 4 & 5 & 6 & 7 & 8 & 9 & 10\end{array}$

1 asp arg VAl tyr val his pro phe his leu ***

\section{COMPOSITION}

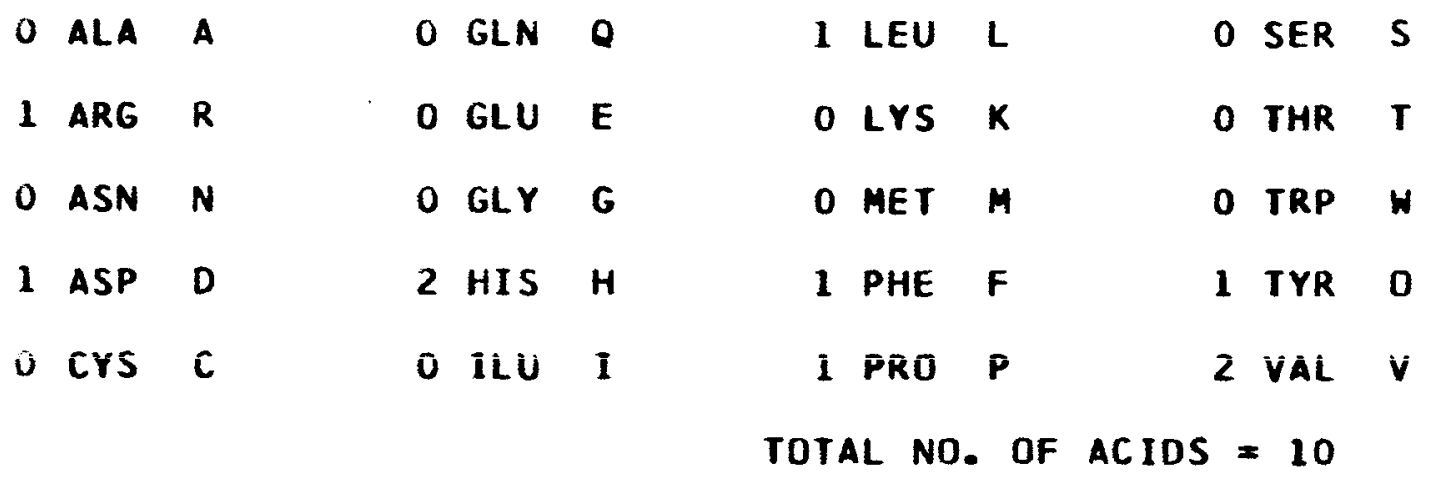

- elliot, D. F., and peart, H. S., BIOChem. J., Vol.65, PP. 246-254, 1957 
ALPha melanocyte-stimulating hoRmone - bOVINE, PIG, and horse

ACETYL AT AMINO END.

C-TERMINAL VALINE IS AMINATED.

1234567890123

SOS MEHFRHGKPV*

$\begin{array}{lllllllllllll}1 & 2 & 3 & 4 & 5 & 6 & 7 & 8 & 9 & 10 & 11 & 12 & 13\end{array}$

SER TYR SER MET GLU HIS PHE ARG TRP GLY LYS PRO VAL * *

\section{COMPOSITION}

\begin{tabular}{|c|c|c|c|c|c|c|c|c|c|c|}
\hline 0 & ALA & A & 0 & GLN & $Q$ & 0 & LEU & $L$ & 2 & SER \\
\hline 1 & ARG & $\mathbf{R}$ & 1 & GLU & $\mathbf{E}$ & 1 & LYS & $K$ & 0 & THR \\
\hline 0 & ASN & $\mathbf{N}$ & 1 & GLY & $\mathbf{G}$ & 1 & MET & $M$ & 1 & TRP \\
\hline 0 & ASP & D & 1 & HIS & $H$ & 1 & PHE & $F$ & 1 & TYR \\
\hline $\mathbf{U}$ & CYS & $\mathrm{C}$ & 0 & $I L U$ & I & 1 & PRO & $\mathbf{P}$ & 1 & VAL \\
\hline
\end{tabular}

- HARRIS, J. I. AND LERNER, A. B., NATURE, VOL.179, NO.4574, PP. 1346-1347, JUNE 29, 1957 (PIG)

LI, C. H., LABORATORY INVESTIGATION, VOL. 8, NO.2, PP. 574-587, 1959 (BOVINE)

DIXON, J. S. AND LI, C. H., J. AM. CHEM. SOC., VOL.82, PP.4568-4572, SEPT. 5, 1960 (HORSE) 
beta melandcyte-stimulating hormone - bovine

123456789012345678

1 DS GP DKMEHFRWGSPPKD

$\begin{array}{lllllllllllllll}1 & 2 & 3 & 4 & 5 & 6 & 7 & 8 & 9 & 10 & 11 & 12 & 13 & 14 & 15\end{array}$

1 ASP SER GLY PRO TYR LYS MET GLU HIS PHE ARG TRP GLY SER PRO PRO LYS ASP ***

\begin{tabular}{|c|c|c|c|c|c|c|c|c|c|c|c|}
\hline & & & & & APC & & & & & & \\
\hline 0 & ALA & $A$ & 0 & GLN & 0 & 0 & LEU & $L$ & 2 & SER & $S$ \\
\hline 1 & ARG & $\mathbf{R}$ & 1 & GLU & $\mathbf{E}$ & 2 & LYS & K & 0 & THR & $\mathbf{T}$ \\
\hline 0 & $A S N$ & $\mathbf{N}$ & 2 & GLY & G & 1 & MET & $M$ & 1 & TRP & $W$ \\
\hline 2 & ASP & D & $\mathbf{I}$ & HIS & H & 1 & PHE & $F$ & 1 & TYR & 0 \\
\hline 0 & CYS & C & 0 & ILU & I & 3 & PRO & $P$ & 0 & VAL & $\mathbf{v}$ \\
\hline
\end{tabular}

- geschWIND, I.I., LI, C. H., and barnafi, l., J. am. Chem. SOC., VOL. 79, PP.1003-1004, FEB. 20, 1957 
Beta melanocyte-stimulating hoRmone - PIg

123345677899012345678

I DEG P DKMEHFRWGSPPKD*

$\begin{array}{lllllllllllllll}1 & 2 & 3 & 4 & 5 & 6 & 7 & 8 & 9 & 10 & 11 & 12 & 13 & 14 & 15\end{array}$

I ASP GLU GLY PRO TYR LYS MET GLU HIS PHE ARG TRP GLY SER PRO PRO LYS ASP *E:

\section{COAPOSITION}

\begin{tabular}{|c|c|c|c|c|c|c|c|c|c|c|}
\hline 0 & ALA & A & 0 & GLN & $\mathbf{Q}$ & 0 & LEU & $L$ & 1 & SER \\
\hline 1 & ARG & $\mathbf{R}$ & 2 & GLU & $E$ & 2 & LYS & $\mathbf{k}$ & 0 & THR \\
\hline 0 & ASN & $\mathbf{N}$ & 2 & GLY & $\mathbf{G}$ & 1 & MET & $M$ & 1 & TRP \\
\hline 2 & ASP & D & 1 & HIS & $H$ & 1 & PHE & $\boldsymbol{F}$ & 1 & TYR \\
\hline 0 & CYS & C & 0 & ILU & $I$ & 3 & PRO & $\mathbf{P}$ & 0 & VAL \\
\hline
\end{tabular}

- HARRIS, J. I. AND ROOS, P., NATURE, VOL.178, NO.4524, P. 90, JULY 14.1956

GESCHWIND, I.I., LI, C. H., AND BARNAFI, L., J. AM. CHEM. SOC.. VOL. 79, PP.620-625, FEB. 5, 1957 
BetA MELANOCYTE-STIMULATING hORMONE - hORSE

123456789012345678

I DEGPOKMEHFRWGSPRKD.

$\begin{array}{lllllllllllllll}1 & 2 & 3 & 4 & 5 & 6 & 7 & 8 & 9 & 10 & 11 & 12 & 13 & 14 & 15\end{array}$

1 ASP GLU GLY PRO TYR LYS MET GLU HIS PHE ARG TRP GLY SER PRO ARG LYS ASP ***

COMPOSITION

o AlA A

O GLN Q

0 LEU L

1 SER $S$

2 ARG R

2 GLU E

2 LYS $K$

O THR T

O ASN N

2 GLY 6

1 MET $M$

1 TRP W

2 ASP D

1 HIS H

1 PHE $F$

1 TYR 0

0 CYS C

0 ILU I

2 PREO P

O VAL $V$

TOTAL NO. OF ACIDS $=18$

- DIXON, J. S. AND LI, C. H., GEN. COMP. ENDOCRINOL.,

VOL.1, PP.161-169, 1961 
Beta melandCyte-stimulatimg hoRmone - human

1234567890123456789012

I AEKKDEGPORMEHFRWGSPPKD.

$\begin{array}{lllllllllllllll}1 & 2 & 3 & 4 & 5 & 6 & 7 & 8 & 9 & 10 & 11 & 12 & 13 & 14 & 15\end{array}$

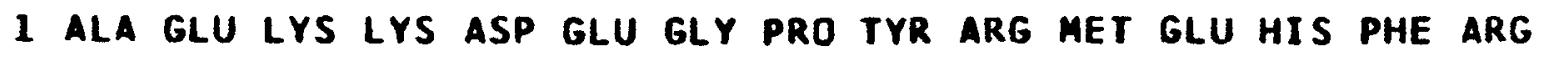
TRP GLY SER PRO PRO LYS ASP ***

\section{COMPOSITION}

\begin{tabular}{|c|c|c|c|c|c|c|c|c|c|}
\hline 1 & ALA & $A$ & 0 & GLN & 0 & 0 LEU & $\mathbf{L}$ & 1 & SER \\
\hline 2 & ARG & $\mathbf{R}$ & 3 & GLU & $E$ & 3 LYS & $K$ & 0 & THR \\
\hline 0 & ASN & $\mathbf{N}$ & 2 & GLY & $\mathbf{G}$ & 1 MET & $M$ & 1 & TRP \\
\hline 2 & ASP & D & 1 & HIS & H & 1 PHE & $F$ & 1 & TYR \\
\hline & CYS & C & 0 & ILU & I & 3 PRO & $\mathbf{P}$ & 0 & VAL \\
\hline
\end{tabular}

- HARRIS, J. I., NATURE, VOL. 184, NO. 4681, PP.167-169, JULY 18,1959 
BETA CORTICOTROPIN - PIG

123456789012345678901234567890

IS DSMEHFRHGKPVGKKRRPVKVDPGAE D D Q 31 L A E F P LEF

$\begin{array}{lllllllllllllll}1 & 2 & 3 & 4 & 5 & 6 & 7 & 8 & 9 & 10 & 11 & 12 & 13 & 14 & 15\end{array}$

I SER TYR SER MET GLU HIS PHE ARG TRP GLY LYS PRO VAL GLY LYS

LYS ARG ARG PRO VAL LYS VAL TYR PRO GLY ALA GLU ASP ASP GLN

31 Leu ala glu ala phe pro leu glu phe ***

\section{COMPOSITION}

\begin{tabular}{|c|c|c|c|c|c|c|c|c|c|c|}
\hline 3 & ALA & $\mathbf{A}$ & 1 & GLN & 0 & 2 & LEU & $L$ & 2 & SER \\
\hline 3 & ARG & $\mathbf{R}$ & 4 & GLU & $\mathbf{E}$ & 4 & LYS & $\mathbf{K}$ & 0 & THR \\
\hline 0 & ASN & $\mathbf{N}$ & 3 & GLY & $\mathbf{G}$ & 1 & MET & $M$ & 1 & TRP \\
\hline 2 & ASP & D & 1 & HIS & $H$ & 3 & PHE & $\mathbf{F}$ & 2 & TYR \\
\hline & Crs. & $c$ & $\overline{0}$ & $\mathbf{I} \mathbf{L} \mathbf{U}$ & I & 4 & PRU & $\mathbf{P}$ & 3 & VAL \\
\hline
\end{tabular}

TOTAL NO. OF ACIDS $=39$

- hHITE, W. F.. AND LANDMANN, H. A., J. AM. CHEM. SOC.. VOL. 77,. PP.1711-1712, MARCH 20, 1955

HOWARD, K. S., SHEPHERD, R. G., EIGNER, E. A., DAVIS, D. S., AND BELL, P. H., J. AM. CHEM. SOC., VOL.77, PP.3419-3420, JUNE 20,1955

BELL, P. H., J. AM. CHEM. SOC., VOL.76, PP.5565-5567, NOV. 1954

THIS SEQUENCE DIFFERS FROM THAT SHOWN ABOVE BY REMOVING THE ASP (D) FROM POSITION 29 AND INSERTING IT BETHEEN POSITIONS 24 AND 25. 
ALPHA CORTICOTROPIN - SHEEP AND BOVINE

123456789012345678901234567890 I S OSMEHFRWGKPVGKKRRPVKVOPDGEAED 31 SA QAFPLEF.

$\begin{array}{lllllllllllllll}1 & 2 & 3 & 4 & 5 & 6 & 7 & 8 & 9 & 10 & 11 & 12 & 13 & 14 & 15\end{array}$

1 SER TYR SER MET GLU HIS PHE ARG TRP GLY LYS PRO VAL GLY LYS LYS ARg ARg PRo VAL LYS YAL TYR PRo ASP gLY GLU ALA GLU ASP 31 Ser ala gln ala phe pro leu glu phe ****

\section{COMPOSITION}

\begin{tabular}{|c|c|c|c|c|c|c|c|c|c|}
\hline 3 ALA & A & 1 & GLN & $Q$ & 1 & LEU & $\mathbf{L}$ & 3 & SER \\
\hline ARG & $\mathbf{R}$ & 4 & GLU & $E$ & 4 & LYS & $\mathbf{K}$ & 0 & THR \\
\hline ASN & $\mathbf{N}$ & 3 & GLY & $\mathbf{G}$ & 1 & MET & $M$ & 1 & TRP \\
\hline ASP & $D$ & 1 & HIS & $H$ & 3 & PHE & $F$ & 2 & TYR \\
\hline crs & C & 0 & ILU & I & 4 & PRO & $\mathbf{P}$ & 3 & VAL \\
\hline
\end{tabular}

TOTAL NO. OF ACIDS $=39$

- LI, C.H., GESCHHIND, I. I., COLE, D., RAACK, I. D., HARRIS, J.I., AND DIXÓN, J. S., NATURE, VOL.176, NO.4484, PP.687-689, OCT. 8, 1955 (SHEEP)

LI, C. H., DIXON, J. S., AND CHUNG, D., J. AM. CHEM. SOC., VOL. $80, P .2587,1958$ (BOVINE) 
INSULIN A - BOVINE

123456789012345678901

1 GIVEQCCASVCSLOQLENOCN.

$\begin{array}{lllllllllllllll}1 & 2 & 3 & 4 & 5 & 6 & 7 & 8 & 9 & 10 & 11 & 12 & 13 & 14 & 15\end{array}$

1 GLY ILU VAL GLU GLN CYS CYS ALA SER VAL CYS SER LEU TYR GLN LEU GLU ASN TYR CYS ASN ***

\section{COMPOSITION}

\begin{tabular}{|c|c|c|c|c|c|c|c|c|c|}
\hline 1 & ALA & A & 2 & GLN & $Q$ & 2 LEU & $\mathbf{L}$ & 2 & SER \\
\hline D & ARG & $\mathbf{R}$ & 2 & GLU & $E$ & O LYS & $K$ & 0 & THR \\
\hline 2 & $A S N$ & $\mathbf{N}$ & 1 & GLY & $\mathbf{G}$ & O MET & $M$ & 0 & TRP \\
\hline D & ASP & D & 0 & HIS & $H$ & O PHE & $F$ & 2 & TYR \\
\hline & Crs & C & 1 & ILU & I & O PRD & $P$ & 2 & VAL \\
\hline
\end{tabular}

- SANGER, F. AND THOMPSON, E. O. P., BIOCHEM J.. VOL.53, PP. 353-374, 1953

THE AMIDE GROUPS WERE SUBSEQUENTLY DETERMINED.

RYLE, A. P., SANGER, F., SMITH, L.F., AND KITAL, R., BIOCHEM. J., VOL. 60, PP. 541-556, 1955 
INSULIN A - BONITO

123456789012345678901

$1 G I(H, E, E, C(C, K, P, H) C, D, L) F$ LE D DCN •

$\begin{array}{lllllllllllllll}1 & 2 & 3 & 4 & 5 & 6 & 7 & 8 & 9 & 10 & 11 & 12 & 13 & 14 & 15\end{array}$

1 GLY ILUIHIS,GLU,GLU, CYSICYS,LYS,PRO, HISICYS, ASP, LEU) PHE GLU LEU GLU ASP TYR CYS ASN ***

\begin{tabular}{|c|c|c|c|c|c|c|c|c|c|c|c|}
\hline \multicolumn{12}{|c|}{ COMPOSITION } \\
\hline 0 & ALA & $A$ & 0 & GLN & 0 & 2 & LEU & $L$ & 0 & SER & $S$ \\
\hline 0 & ARG & $R$ & 4 & GLU & $E$ & 1 & LYS & K & 0 & THR & $\mathbf{T}$ \\
\hline 1 & ASN & $N$ & 1 & GLY & G & 0 & MET & $M$ & 0 & TRP & $W$ \\
\hline 2 & ASP & D & 2 & HIS & H & 1 & PHE & $\mathbf{F}$ & 1 & TYR & 0 \\
\hline 4 & crs & C & 1 & ILU & I & $\mathbf{1}$ & PRO & $\mathbf{P}$ & 0 & VAL & V \\
\hline
\end{tabular}

* KOTAKI, A., J. BIOCHEM. (TOKYO), VOL.53, NO.1, PP.61-70, 1963 


\section{INSULIN A - HORSE}

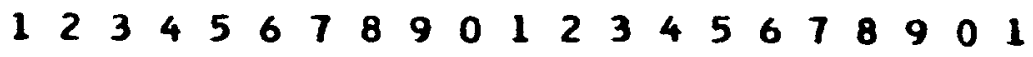

I GIVEACCTGICSLOQLENOCN

$\begin{array}{lllllllllllllll}1 & 2 & 3 & 4 & 5 & 6 & 7 & 8 & 9 & 10 & 11 & 12 & 13 & 14 & 15\end{array}$

I GLY ILU VAL GLU GLN CYS CYS THR GLY ILU CYS SER LEU TYR GLN LEU GLU ASN TYR CYS ASN ***

\section{COMPOSITION}

\begin{tabular}{|c|c|c|c|c|c|c|c|c|c|}
\hline O ALA & $\mathbf{A}$ & 2 & GLN & 0 & 2 & LEU & $\mathbf{L}$ & 1 & SER \\
\hline O ARG & $\mathbf{R}$ & 2 & GLU & $E$ & 0 & LYS & $\mathbf{K}$ & 1 & THR \\
\hline $2 A S N$ & $\mathbf{N}$ & 2 & GLY & $\mathbf{G}$ & 0 & MET & $M$ & 0 & TRP \\
\hline ASP & D & 0 & HIS & $H$ & 0 & PHE & $\mathbf{F}$ & 2 & TYR \\
\hline CYS & C & 2 & ILU & I & 0 & PRO & $\mathbf{P}$ & 1 & VAL \\
\hline
\end{tabular}

- HARR IS, J. I., SANGER, F., AND NAUGHTON, M. A., ARCH. BIOCHEM. BIOPHYS., VOL.65, PP.427-438, 1956

SOME EVIDENCE FOR THE SEQUENCE HAS DERIVED FROM HOMOLOGY WITH BOVINE INSULIN. 
INSULIN A - SHEEP

1234567899012345678901

I G IVEOCCAGVCSLOQLENOCN.

$\begin{array}{lllllllllllllll}1 & 2 & 3 & 4 & 5 & 6 & 7 & 8 & 9 & 10 & 11 & 12 & 13 & 14 & 15\end{array}$

1 gLY ILU YAL GLU GLN CYS CYS ALA gLY VAL CYS SER LEU TYR GLN

LEU GLU ASN TYR CYS ASN

COMPOSITION

\begin{tabular}{|c|c|c|c|c|c|c|c|c|c|c|}
\hline 1 & ALA & $\mathbf{A}$ & 2 & GLN & 0 & 2 & LEU & $\mathbf{L}$ & 1 & SER \\
\hline 0 & ARG & $\mathbf{R}$ & 2 & GLU & $E$ & 0 & LYS & $k$ & 0 & THR \\
\hline 2 & ASN & $\mathbf{N}$ & 2 & GLY & G & 0 & MET & $\boldsymbol{M}$ & 0 & TRP \\
\hline 0 & ASP & D & 0 & HIS & $H$ & 0 & PHE & $F$ & 2 & TYR \\
\hline 4 & CYS & C & 1 & ILU & I & 0 & PRO & $\mathbf{P}$ & 2 & VAL \\
\hline
\end{tabular}

TOTAL NO. OF ACIDS $=21$

- BROWN, H., SANGER, F., AND KITAI, R., BIOCHEM. J., VOL.60, PP. 556-565, 1955

SOME EVIDENCE FOR THE SEQUENCE HAS DER IVED FROM HOMOLOGY WITH BOVINE INSULIN. 
INSULIN A - SPERA WHALE, FIN-HHALE, PIG, AND HUMAN

123456789012345678901

I GIVEQCCTSICSLOOLENOCN.

$\begin{array}{lllllllllllllll}1 & 2 & 3 & 4 & 5 & 6 & 7 & 8 & 9 & 10 & 11 & 12 & 13 & 14 & 15\end{array}$

1 GLY ILU VAL GLU GLN CYS CYS THR SER ILU CYS SER LEU TYR GLN LEU GLU ASN TYR CYS ASN *.*

COMPOSITION

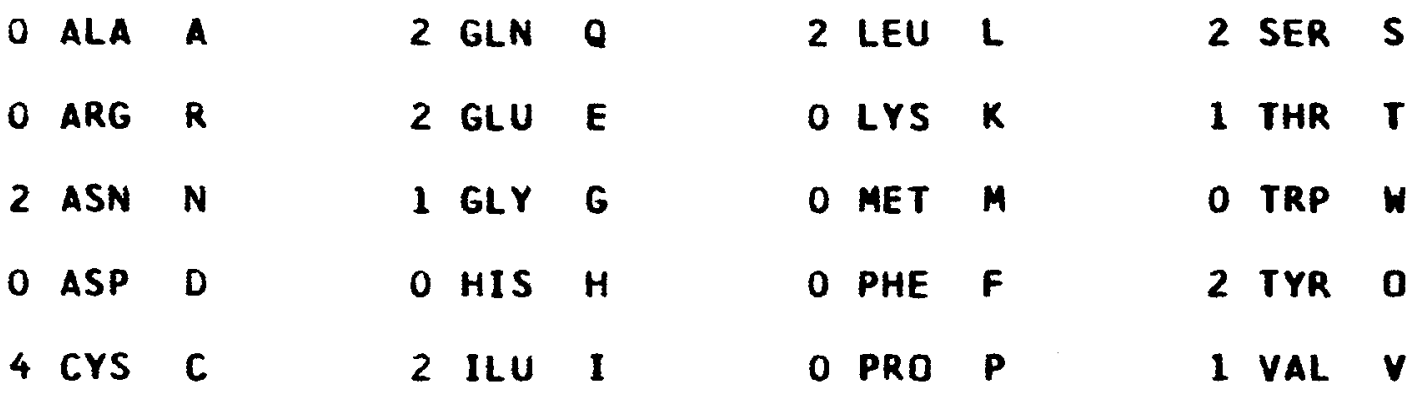

TOTAL NO. OF ACIDS $=21$

- BROWN, h., SANGer, F., AND KITAI, R., BIOCHEM. J., VOl.60, PP.556-565, 1955 (PIG)

SUME EVIDENCE FOR THE SEQUENCE WAS DERIVED FROM HOMOLOGY WITH BOVINE INSULIN.

HARR IS, J. I., SANGER, F., AND NAUGHTON, M. A., ARCH. BIOCHEM. BIOPHYS., VOL.65, PP.427-438, 1956 (SPERM WHALE)

HAMA, H., TITANI, K., SAKAKI, S., AND NARITA, K., J. BIOCHEM. (TOKYO), VOL.56, NO.3, PP.285-293, 1964 (FIN-WHALE)

THIS WORK CONFIRMED THE SEQUENCE ABOVE, EXCEPT GLU (E) AND GLN (O) WERE INTERCHANGED AT POSITIONS 15 AND 17.

NICOL, D. S. H. AND SHITH, L. F., NATURE, VOL. 187, N0.4736, PP. 483-485, AUG. 6, 1960 (HUMAN) 


\section{INSULIN A - SEI-WHALE}

12345678901234567789901

I G I V E Q C C A S T C SLOOLENOCN

$\begin{array}{lllllllllllllll}1 & 2 & 3 & 4 & 5 & 6 & 7 & 6 & 9 & 10 & 11 & 12 & 13 & 14 & 15\end{array}$

1 GLY ILU VAL GLU GLN CYS CYS ALA SER THR CYS SER LEU TYR GLN

LEU GLU ASN TYR CYS ASN *:*

\section{COMPOSITION}

\begin{tabular}{|c|c|c|c|c|c|c|c|c|c|}
\hline ALA & $A$ & 2 & GLN & 0 & 2 & LEU & $L$ & 2 & SER \\
\hline ARG & $\mathbf{R}$ & 2 & GLU & $\mathbf{E}$ & 0 & LYS & $K$ & 1 & THR \\
\hline 2 ASN & $\mathbf{N}$ & 1 & GLY & $\mathbf{G}$ & 0 & MET & $M$ & 0 & TRP \\
\hline ASP & D & 0 & HIS & $H$ & 0 & PHE & $F$ & 2 & TYR \\
\hline CYS & $C$ & 1 & ILU & I & 0 & PRO & $\mathbf{P}$ & 1 & VAL \\
\hline
\end{tabular}

ISHIHARA, Y.. SAITO, T., ITO, Y.. AND FUJINO, M., NATURE, VOL.181, NO.4621, PP.1461-1469, MAY 24, 1958 (SE I-WHALE) 
INSULIN B - BOVINE, SHEEP, hORSE, hUMAN, PIG, AND SPERM hHALE

TWO DISULPHIDE BDNDS CONNECT THE A ANO B CHAINS.

A7 IS BONDED TO B7 AND A20 IS BONDED TO B19. IN ADDITION THERE

IS A BOND FROM AG TO All.

123456789012234567889011234567896

I FVN Q H L C G H L VEALOLV CGERGFFOTAKA

$\begin{array}{lllllllllllllll}1 & 2 & 3 & 4 & 5 & 6 & 7 & 8 & 9 & 10 & 11 & 12 & 13 & 14 & 15\end{array}$

1 phe val asN gLN his Leu Cys gLy Ser his Leu val glu ala leu TYR LEU VAL CYS GLY GLU ARG GLY PHE PHE TYR THR PRO LYS ALA

\section{COMPOSITION}

\begin{tabular}{|c|c|c|c|c|c|c|c|c|c|c|}
\hline 2 & ALA & A & 1 & GLN & 0 & 4 & LEU & $\mathbf{L}$ & 1 & SER \\
\hline 1 & ARG & $\mathbf{R}$ & 2 & GLU & $E$ & 1 & LYS & $\mathbf{K}$ & 1 & THR \\
\hline 1 & ASN & $\mathbf{N}$ & 3 & GLY & G & 0 & MET & $M$ & 0 & TRP \\
\hline 0 & ASP & D & 2 & HIS & $\mathrm{H}$ & 3 & PHE & $F$ & 2 & TYR \\
\hline 2 & CYS & $c$ & 0 & ILU & I & 1 & PRO & $\mathbf{P}$ & 3 & VAL \\
\hline
\end{tabular}

TOTAL NO. OF ACIDS $=30$

- RYle, A. P., SANGer, F., SMITH, L.F., AND KITAI, R., BIOCHEM. J., VOL. 60, PP.541-556, 1955 (BDVINE, SHEEP, AND PIG)

SANGER, F. AND TUPPY, H., BIOCHEM. J., VDL.49, PP.481-490, 1951 (BCVINE)

THE AMIDE GROUPS WERE SUBSEQUENTLY DETERMINED.

HARRIS, J. I., SANGER, F., AND NAUGHTON, M. A., ARCH. BIOCHEM. BIOPHYS., VOL-65, PP. $427-438,1956$ (SPERM WHALE AND HORSE)

ISHIHARA, Y., SAITO, T., ITO, Y., AND FUJINO, M., NATURE, YOL.181, NO.4621, PP.1461-1469, MAY 24,1958 (SPERM AND SEI-WHALE)

NICOL, D. S. H. AND SMITH, L.F., NATURE, VUL.187, NO.4736, PP.483-485, AUG. 6, 1960 (HUAAN)

HUMAN INSUL IN B CHAIN IS IDENTICAL WITH ABOVE EXCEPT THAT POSITION 30 IS THR (T). 
INSULIN B - BONITO

123456789012344567899012234567890 1 A A N(P,H,LICIG,S,H,L,V,E,A,L)OL(V,C,G,E)R GFF O Q P K

$\begin{array}{lllllllllllllll}1 & 2 & 3 & 4 & 5 & 6 & 7 & 8 & 9 & 10 & 11 & 12 & 13 & 14 & 15\end{array}$

1 aLA ALA ASN(PRO,HIS,LEUICYSIGLY,SER, HIS,LEU, VAL, GLU, ALA, LEU) TYR LEUIVAL, CYS, GLY, GLUiARg GLY PHE PHE TYR GLN PRO LYS **

COMPOSITION

\begin{tabular}{|c|c|c|c|c|c|c|c|c|c|c|}
\hline 3 & ALA & A & 1 & GLN & 0 & 4 & LEU & $\mathbf{L}$ & 1 & SER \\
\hline 1 & ARG & $\mathbf{R}$ & 2 & GLU & $E$ & 1 & LYS & $k$ & 0 & THR \\
\hline 1 & ASN & $N$ & 3 & GLY & $\mathbf{G}$ & 0 & MET & $M$ & 0 & IRP \\
\hline 0 & ASP & D & 2 & HIS & H & 2 & PHE & $F$ & 2 & TYR \\
\hline$<$ & CYS & $C$ & 0 & ILU & I & 2 & PRO & $\mathbf{P}$ & 2 & VAL \\
\hline
\end{tabular}

- KOTAKI, A., J. BIOCHEM. (TOKYO), VÓL.51, N0.4, PP.301-309, 1962 
FIBR INOPEPTIDES ARE THOSE PORTIONS OF VERTEBRATE FIBRINOGEN MOLECULES WHICH ARE PROTEOLYTICALLY REMOVED BY THE ENZYME THROMBIN. THEIR REMOVAL PERMITS SPONTANEOUS POLYMERIZATION DF THE PARENT MOLECULES TO FORM AM INSOLUBLE FIBRINOGEL. SINCE THE FUNCTION OF THE FIBR INOPEPTIDES IS RATHER NON-SPECIFIC, LARGE SEQUENCE CHANGES ARE OBSERVED AMONG CLOSELY RELATED SPECIES.

12344567789012234567789

1 EDGS DPPSGDFLTEGGGVR/

$\begin{array}{lllllllllllllll}1 & 2 & 3 & 4 & 5 & 6 & 7 & 8 & 9 & 10 & 11 & 12 & 13 & 14 & 15\end{array}$

1 GLU ASP GLY SER ASP PRO PRO SER GLY ASP PHE LEU THR GLU GLY GLY GLY VAL ARG ///

\section{COMPOSITION}

\begin{tabular}{|c|c|c|c|c|c|c|c|c|c|c|}
\hline 0 & ALA & $A$ & 0 & GLN & 0 & 1 & LEU & $L$ & 2 & SER \\
\hline 1 & $A R G$ & $\mathbf{R}$ & 2 & GLU & $\vec{E}$ & 0 & LYS & $\mathbf{K}$ & I & THK \\
\hline 0 & ASN & $\mathbf{N}$ & 5 & GLY & $\mathbf{G}$ & 0 & MET & $\mathbf{M}$ & 0 & TRP \\
\hline 3 & ASP & $\mathrm{D}$ & 0 & HIS & $H$ & 1 & PHE & $F$ & 0 & TYR \\
\hline & CYS & $C$ & 0 & ILU & I & 2 & PRD & $\mathbf{P}$ & 1 & VAL \\
\hline
\end{tabular}

- DOOLITTLE, R. F. AND BLOMBACK, B., NATURE, VOL. 202, NO. 4928, PP. 147-152, APRIL 11, 1964 
FIBR INOPEPTIDE A - SHEEP

123456678901223456789

1 A D DS DPVGGEFLAEGGGVR,

$\begin{array}{lllllllllllllll}1 & 2 & 3 & 4 & 5 & 6 & 7 & 8 & 9 & 10 & 11 & 12 & 13 & 14 & 15\end{array}$

1 ALA ASP ASP SER ASP PRO VAL GLY GLY GLU PHE LEU ALA GLU GLY GLY GLY VAL ARG ///

\section{COMPOSITION}

\begin{tabular}{|c|c|c|c|c|c|c|c|c|c|}
\hline ALA & $A$ & 0 & GLN & $Q$ & 1 & LEU & $L$ & 1 & SER \\
\hline ARG & $\mathbf{R}$ & 2 & GLU & $E$ & 0 & LYS & $K$ & 0 & THR \\
\hline$A S N$ & $N$ & 5 & GLY & 6 & 0 & MET & $M$ & 0 & TRP \\
\hline$A S P$ & D & 0 & HIS & $H$ & 1 & PHE & $F$ & 0 & TYR \\
\hline CYS & $c$ & 0 & ILU & I & 1 & PRO & $p$ & 2 & VAL \\
\hline
\end{tabular}

- DOOLITILE, R. F. AND BLOMBACK, B., NATURE, VOL. 202, NO. 4928, PP. 147-152, APR IL 11, 1964 


\section{FIBRINOPEPTIDE A - GDAT}

1234567890123456789

I A D DSDPVGGEFLAEGGGVRI

$\begin{array}{lllllllllllllll}1 & 2 & 3 & 4 & 5 & 6 & 7 & 8 & 9 & 10 & 11 & 12 & 13 & 14 & 15\end{array}$

1 ALA ASP ASP SER ASP PRO VAL GLY GLY GLU PHE LEU ALA GLU GLY GLY GLY VAL ARG ///

\section{COMPOSITION}

\begin{tabular}{|c|c|c|c|c|c|c|c|c|c|}
\hline ALA & A & 0 & GLN & 0 & 1 & LEU & $\mathbf{L}$ & 1 & SER \\
\hline ARG & $\mathbf{R}$ & 2 & GLU & $\mathbf{E}$ & 0 & LYS & $K$ & 0 & THR \\
\hline ASN & $N$ & 5 & GLY & $\mathbf{G}$ & 0 & MET & $M$ & 0 & TRP \\
\hline ASP & D & 0 & HIS & $H$ & 1 & PHE & $\mathbf{F}$ & 0 & IYR \\
\hline crs & c & 0 & ILU & I & 1 & PRO & $\mathbf{P}$ & 2 & VAL \\
\hline
\end{tabular}

- dodlitile, R. F. and blomback, B., nature, vol. 202, NO. 4928, PP. 147-152, APRIL 11,1964 
FIBR INOPEPTIDE A - REINDEER

123445667889012234556789

IA DGS D P A G G E F(L,A,E,G,G,G,V)R /

$\begin{array}{lllllllllllllll}1 & 2 & 3 & 4 & 5 & 6 & 7 & 8 & 9 & 10 & 11 & 12 & 13 & 14 & 15\end{array}$

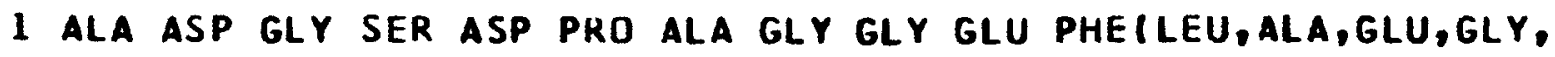
GLY,GLY,VALIARG ///

\section{COMPOSITION}

\begin{tabular}{|c|c|c|c|c|c|c|c|c|c|}
\hline$A L A$ & $A$ & 0 & GLN & $\mathbf{Q}$ & 1 & LEU & $\mathbf{L}$ & 1 & SER \\
\hline ARG & $\mathbf{R}$ & 2 & GLU & $\mathbf{E}$ & 0 & LYS & $K$ & 0 & THR \\
\hline ASN & $\mathbf{N}$ & 6 & GLY & G & 0 & MET & $M$ & 0 & TRP \\
\hline ASP & D & 0 & HIS & $\mathbf{H}$ & 1 & PHE & $\mathbf{F}$ & 0 & TYR \\
\hline CYS & 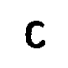 & 0 & ILU & 1 & 1 & PRO & $\mathbf{P}$ & 1 & VAL \\
\hline
\end{tabular}

- doulitTle, R. F. AND Blomback, B., nature, Vol. 202, NO. 4928, PP. 147-152, APR IL 11,1964 
PIBR INOPEPTIDE A - PIG

12334566778990112334567

I A E U DKGEFLAEGGGVR/

$\begin{array}{llllllllllllllll}\cdot & 1 & 2 & 3 & 4 & 5 & 6 & 7 & 8 & 9 & 10 & 11 & 12 & 13 & 14 & 15\end{array}$

I ALA GLU val GLN ASP LYS GLY GLU PHE LEU ALA GLU GLY GLY GLY VAL ARG ///

\section{COMPOSITION}

\begin{tabular}{|c|c|c|c|c|c|c|c|c|c|c|}
\hline 2 & ALA & A & 1 & GLN & $Q$ & 1 & LEU & $\mathbf{L}$ & 0 & SER \\
\hline 1 & ARG & $\mathbf{R}$ & 3 & GLU & $E$ & 1 & LYS & $k$ & 0 & THR \\
\hline 0 & ASN & $\mathbf{N}$ & 4 & GLY & $\mathbf{G}$ & 0 & MET & $M$ & 0 & TRP \\
\hline 1 & ASP & D & 0 & HIS & $H$ & 1 & PHE & $\mathbf{F}$ & 0 & TYR \\
\hline 0 & CYS & $C$ & 0 & ILU & I & 0 & PRO & $\mathbf{P}$ & 2 & VAL \\
\hline
\end{tabular}

* DOOl ItTle, R. F. AND Blomback, B., NATURE, Vol. 202, NO. 4928, PP. 147-152, APRIL 11, 1964 
FIBR INOPEPTIDE A - HUMAN

1234567890123456

IA DSGEGDFLAEGGGVR,

$\begin{array}{lllllllllllllll}1 & 2 & 3 & 4 & 5 & 6 & 7 & 8 & 9 & 10 & 11 & 12 & 13 & 14 & 15\end{array}$

1 aLA ASP SER GLY GLU GLY ASP PHE LEU ALA GLU GLY GLY GLY VAL ARG //

\section{COMPOSITION}

\begin{tabular}{|c|c|c|c|c|c|c|c|c|c|c|}
\hline 2 & ALA & A & 0 & GLN & $Q$ & 1 & LEU & $L$ & 1 & SER \\
\hline 1 & ARG & $\mathbf{R}$ & 2 & GLU & $E$ & 0 & LYS & K & 0 & THR \\
\hline 0 & ASN & $N$ & 5 & GLY & G & 0 & MET & $M$ & 0 & TRP \\
\hline 2 & ASP & $D$ & 0 & HIS & $\mathbf{H}$ & 1 & PHE & $\mathbf{F}$ & 0 & IYR \\
\hline 0 & crs & C & 0 & ILU & I & 0 & PRO & $p$ & 1 & VAL \\
\hline
\end{tabular}

TOTAL ND. OF ACIOS $=16$

- DOOLITILE, R. F. AND BLOMBACK, B., NATURE, VOL. 202, NO. 4928, PP. 147-152, APRIL 11, 1964

PHOSPHD-SERINE OCCURS AT POSITION 3 IN ABOUT HALF THE MOLECULES. A MINOR COMPONENI FRAGMENT, WITH THE N TERMINAL ALANINE MISSING, HAS BEEN DETECTED IN ALL INDIVIDUALS. 


\section{FIBR INOPEPT IDE A - RABBIT}

1234567890123456

IVDPGETSFLL(T,E,G,G)DAR/

$\begin{array}{lllllllllllllll}1 & 2 & 3 & 4 & 5 & 6 & 7 & 8 & 9 & 10 & 11 & 12 & 13 & 14 & 15\end{array}$

I VAL ASP PRO GLY GLU THR SER PHE LEU(THR,GLU,GLY,GLYIASP ALA ARG ///

\section{COMPOSITION}

1 ALA A

O GLN Q

1 LEU L

1 SER $S$

1 ARG $R$

2 GLU E

O LYS K

2 THR T

O ASN N

3 GLY G

O MET $M$

O TRP H

2 ASP D

O HIS H

1 PHE $F$

O TYR 0

o CYS C

O ILU I

1 PRO P

1 VAL V

TOTAL NO. OF ACIDS $=16$

- DOOLITTLE, R. F. AND Blomback, B., NATURE, VOL. 202, NO. 4928, PP. 147-152, APRIL 11, 1964 
FIBRINOPEPTIDE B - BOVINE

PYRROLIDONE CARBOXYLIC ACID - AT AMINO END SO4 ATTACHED TO TYROSINE AT POSITION 5

12345678901234567890

IFPTDODEG OD DRPKVGLGAR/

$\begin{array}{lllllllllllllll}1 & 2 & 3 & 4 & 5 & 6 & 7 & 8 & 9 & 10 & 11 & 12 & 13 & 14 & 15\end{array}$

1 PHE PRO THR ASP IYR ASP GLU GLY GLN ASP ASP ARG PRO LYS VAL GLY LEU GLY ALA ARG //

\section{COMPOSITION}

\begin{tabular}{|c|c|c|c|c|c|c|c|c|c|}
\hline ALA & A & 1 & GLN & 0 & 1 & LEU & $\mathbf{L}$ & 0 & SER \\
\hline ARG & $\mathbf{R}$ & 1 & GLU & $E$ & 1 & LYS & $K$ & 1 & THR \\
\hline ASN & $\mathbf{N}$ & 3 & GLY & $\mathbf{G}$ & 0 & MET & $M$ & 0 & TRP \\
\hline ASP & D & 0 & HIS & H & 1 & PHE & $F$ & 1 & TYR \\
\hline CYS & $C$ & 0 & ILU & I & 2 & PRO & P & 1 & VAL \\
\hline
\end{tabular}

- doolittle, R. F. AND Blomback, B., Nature, VDl. 202, NO. 4928, PP. 147-152, APRIL 11, 1964 
FIBR INOPEPTIDE $B$ - SHEEP

SL 4 ATTACHED TO TYROSINE AT POSITION 5

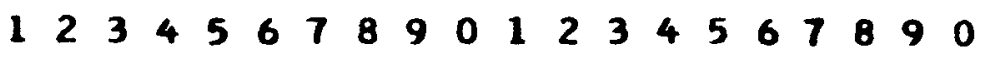

1 G OL L O DEV D D NRAKLPLDAR/

$\begin{array}{lllllllllllllll}1 & 2 & 3 & 4 & 5 & 6 & 7 & 8 & 9 & 10 & 11 & 12 & 13 & 14 & 15\end{array}$

1 GLY TYR LEU ASP TYR ASP GLU VAL ASP ASP ASN ARG ALA LYS LEU PRO LEU ASP ALA ARG $/ / 1$

\section{COMPOSITION}

\begin{tabular}{|c|c|c|c|c|c|c|c|c|c|c|}
\hline 2 & ALA & $A$ & 0 & GLN & 0 & 3 & LEU & $L$ & 0 & SER \\
\hline 2 & ARG & $\mathbf{R}$ & 1 & GLU & $E$ & 1 & LYS & $k$ & 0 & THR \\
\hline l & ASN & $\mathbf{N}$ & 1 & GLY & $G$ & 0 & MET & $M$ & 0 & TRP \\
\hline & ASP & D & 0 & HIS & $H$ & 0 & PHE & $F$ & 2 & TYR \\
\hline & CYS & C & 0 & ILU & 1 & 1 & PRO & $\mathbf{P}$ & 1 & VÀL \\
\hline
\end{tabular}

- DCOLItTle, R. F. AND BlomBaCK, B., NATURE, VOL. 202, NO. 4928, PP. 147-152, APRIL 11, 1964 


\section{FIBR INOPEPTIDE B - GOAT}

SO4 ATTACHED to TYROSINE AT POSITION 5

12345678901234567890

$1 G O L D O D E V D D N R A K L P L D A R /$

$\begin{array}{lllllllllllllll}1 & 2 & 3 & 4 & 5 & 6 & 7 & 8 & 9 & 10 & 11 & 12 & 13 & 14 & 15\end{array}$

1 GLY TYR LEU ASP TYR ASP GLU VAL ASP ASP ASN ARg ALA LYS LEU PKO LEU ASP ALA ARG //

\section{COMPOSITION}

$\begin{array}{lllllllll}2 \text { ALA } & \text { A } & \text { O GLN } & \text { Q } & 3 \text { LEU L } & 0 \text { SER } & S \\ 2 \text { ARG } & \text { R } & 1 \text { GLU } & \text { E } & 1 \text { LYS } & \text { K } & 0 \text { THR } & \text { T } \\ 1 \text { ASN N } & 1 \text { GLY } & \text { G } & 0 \text { MET } & M & 0 \text { TRP } & H \\ 5 \text { ASP } & \text { D } & 0 \text { HIS } & \text { H } & 0 \text { PHE } & \text { F } & 2 \text { TYR } & 0 \\ 0 \text { CYS } & \text { C } & 0 \text { ILU } & \text { I } & 1 \text { PRO P } & 1 \text { VAL } & V \\ & & & \end{array}$

- doOlitTle, R. F. and blomback, B., NatURe, VOL. 202, NO. 4928, PP.147-152, APRIL 11,1964 
FIBRINOPEPTIDE B - REINDEER

PYRROL IDONE CARBOXYLIC ACID - AT AMINO END

SO4 ATIACHED TO TYROSINE AT POSITION 4

A MUTANT HAS BEEN FOUND WHERE GLYCINE REPLACES HISTIDINE IN POSITION 9.

1234567890123456789

I LA DODEV(E,H,D)RAKLHLDAR/

$\begin{array}{lllllllllllllll}1 & 2 & 3 & 4 & 5 & 6 & 7 & 8 & 9 & 10 & 11 & 12 & 13 & 14 & 15^{\circ}\end{array}$

1 LEU ALA ASP IYR ASP GLU VALIGLU,HIS, ASPIARG ALA LYS LEU HIS LEU ASP ALA ARG //

COMPOSITION

\begin{tabular}{|c|c|c|c|c|c|c|c|c|c|c|}
\hline & ALA & $A$ & 0 & GLN & 0 & 3 & LEU & $\mathbf{L}$ & 0 & SER \\
\hline & ARG & $\hat{R}$ & 2 & GLU & $E$ & 1 & IYs & $k$ & 0 & THR \\
\hline & ASN & $N$ & 0 & GLY & 6 & 0 & MET & $M$ & 0 & TRP \\
\hline & ASP & D & 2 & HIS & $H$ & 0 & PHE & $F$ & 1 & TYR \\
\hline & CYS & c & 0 & ILU & I & 0 & PRO & $\mathbf{p}$ & 1 & VAL \\
\hline
\end{tabular}

TOTAL NO. OF ACIDS $=19$

- doolittle, r. F. and blomback, B., nature, vol. 202, NO. 4928, PP. 147-152, APRIL 11, 1964 


\section{FIBR INOPEPTIDE B - PIG}

SO4 ATTACHED TO TYROSINE AT POSITION 4

1233456778901234456789

I A I D O DE DE D GRPKVHVDAR/

$\begin{array}{lllllllllllllll}1 & 2 & 3 & 4 & 5 & 6 & 7 & 8 & 9 & 10 & 11 & 12 & 13 & 14 & 15\end{array}$

I ALA ILU ASP TYR ASP GLU ASP GLU ASP GLY ARG PRO LYS VAL HIS VAL ASP ALA ARG ///

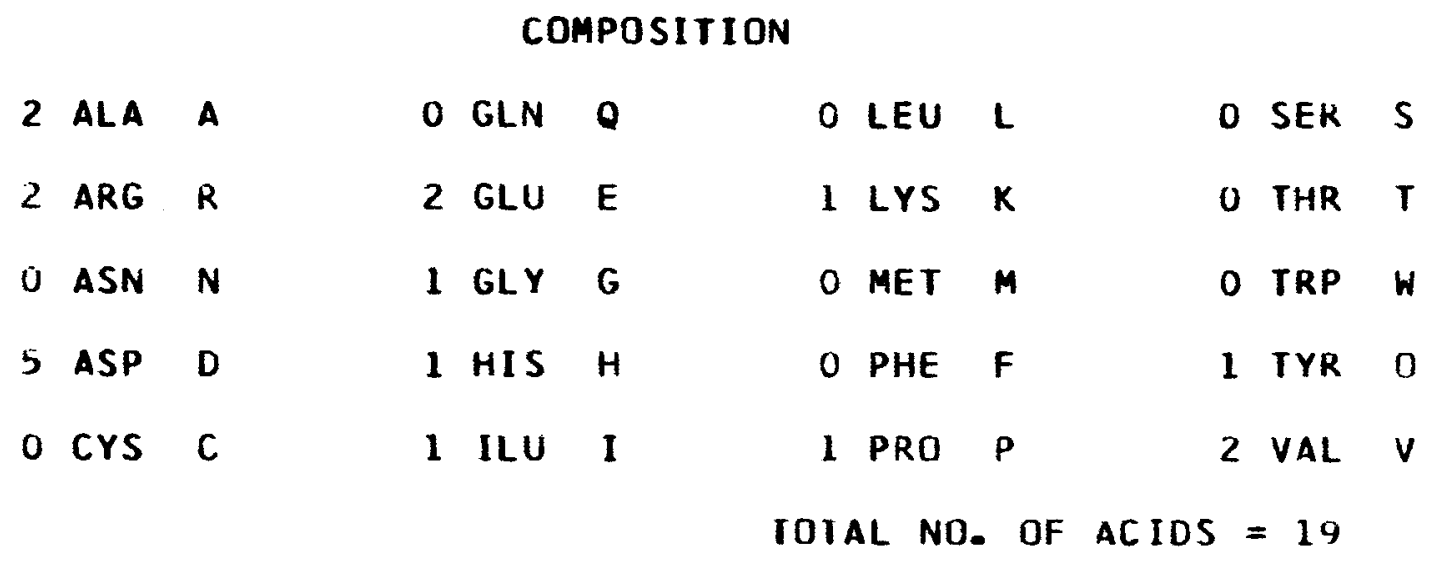

- DoOlittle, R. F. AND Blomback, B.. NATURE, VOl. 202 , NO. 4928, PP. 147-152, APRIL 11, 1964 
FIBRINOPEPTIDE B - HUMAN

PYRROLIDONE CARBOXYLIC ACID - AT AMINO END PHOSPHO-SERINE OCCURS IN POSITION 11.

1234567890123

I G VNDNEEGFFSAR/

$\begin{array}{lllllllllllll}1 & 2 & 3 & 4 & 5 & 6 & 7 & 8 & 9 & 10 & 11 & 12 & 13\end{array}$

1 GLY VAL ASN ASP ASN GLU GLU GLY PHE PHE SER ALA ARg //

COMPOSITION

1 ALA A

1 ARG $R$

2 ASN N

1 ASP D

0 CYS C
O GLN 0

2 GLU E

2 GLY G

O HIS H

0 ILU I
0 LEU L

O LYS K

O MET M

2 PHE $F$

O PRO P

TOIAL NO. DF ACIDS $=13$
1 SER $S$

O THR T

0 TRP $W$

o tYr 0

1 VAL $V$

- DOOlitTle, R. F. AND blomback, B., NATURE, VDl. 202, ND. 4928, PP. 147-152, APRIL 11,1964 


\section{FIBRINOPEPTIDE B - RABBIT}

SO 4 ATTACHED TO TYROSINE AT POSITION 4

1234567890123

IA D D OID,E,P,L,O,VID A R /

$\begin{array}{lllllllllllll}1 & 2 & 3 & 4 & 5 & 6 & 7 & 8 & 9 & 10 & 11 & 12 & 13\end{array}$

1 ALA ASP ASP TYRIASP,GLU,PRO,LEU,ASP, VAL)ASP ALA ARG //

\section{COMPOSITION}

\begin{tabular}{|c|c|c|c|c|c|c|c|c|c|c|}
\hline & ALA & $A$ & 0 & GLN & 0 & 1 & LEU & $\mathbf{L}$ & 0 & SER \\
\hline & ARG & $\mathbf{R}$ & 1 & GLU & $\mathbf{E}$ & 0 & LYS & $k$ & 0 & THR \\
\hline & ASN & $N$ & 0 & GLY & $\mathbf{G}$ & 0 & MET & $M$ & 0 & TRP \\
\hline & ASP & D & 0 & HIS & $H$ & 0 & PHE & $F$ & 1 & TYR \\
\hline & CYS & C & 0 & ILU & $\mathbf{I}$ & 1 & PRO & $\mathbf{P}$ & 1 & VAL \\
\hline
\end{tabular}

- dColitille, R. F. and blomback, B., Nature, vol. 202, NO. 4928, PP. 147-152, APRIL 11, 1964 
Immunoglobulins are serum proteins distinguishable by electrophoretic mobilities, sedimentation coefficients and differential solubilities in variable ethanol-salt solutions. Of these, the gamma globulins are associated with normal antibody function. A proposal for the structure of gamma globulin has been made by Porter (1959) and Fleishman et al, (1963).

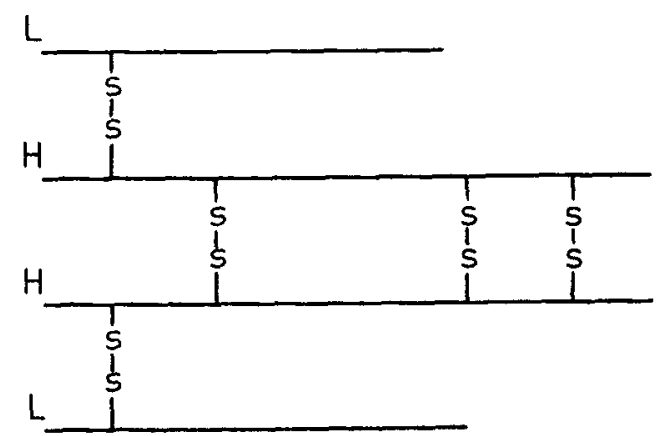

Gamma globulin is thought to be a tetramer consisting of two pairs of identical polypeptide chains held in a particular configuration by disulfide bonds. There are two L (m.w. 20-25,000 each) and two $H$ chains (m.w. 50,000-55,000 each). Because of the chemical problems associated with elucidation of gamma globulin structure, attention has turned to the abundantly produced, structurally similar giobuitins found in multiple myeloma.

Bence-Jones proteins are found exclusively in the urine of all multiple myeloma patients, and probably represent abnormal protein synthesized by the multiple myeloma tumor cell. They are thought to be made exclusively of $L$ chains, related to gamma globulins, (Edelman and Gally, 1962, S. Cohen, 1963, Putnam 1962). It is thought that determination of the amino acid sequence of a particular individual's Bence-Jones protein would reflect a homologous sequence in that individual's antibody structure, thereby partially elucidating the structure of gamma globulin.

Cohen, S. Biochem. J. Vol. 89, p. 334 (1963)

Edelman, G. M. and Gally, J. A. J. Exp. Med. Vol. 116, p. 207 (1962)

Fleishman, J. B. et al. Biochem. J. Vol. 88, p. 220 (1963)

Porter, R. R. Biochem. J. Vol. 73, p. 119 (1959)

Putnam, F. W. Biochim. Biophys. Acta. Vol. 63, p. 539 (1962) 
BENCE-JONES PROTEIN

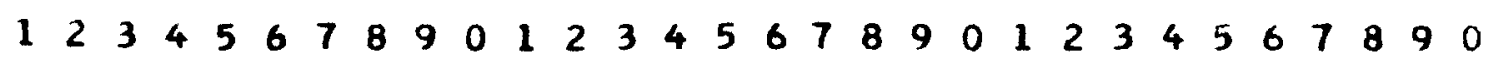

$1 D(T, S, S, S, E, E, P, M, I) L S(S, G, A, V) D R(D, T, T, S, S, E, E, A, V, I, I, I$, $31 F, C) L(0, D, W, E, E, P, G) K K A P K L L I O D A S K L E(S, P, G, A, V)$ 61 R F S(D,T,T,S,G,G,G)F T(D,S,S,E,E,P,I,L)I A T OID,D,T,E,E,P,

$91 L, L, C, O, F, F) G(T, G, G) K \vee D F K R T(S, P, A, A, V) \vee F I(D, S, E, E, P$, $121 P, F) L K S(T, S, G, A) V(V, C) L L D(D, P, F) O R E A K V E W K V(D, D$, $151 D, S, S, E, E, G, A, L) E S(D, T, S, E, E, V) K D(T, S) 0 S S S T L L T L S$ IU1 K A D O E K H K L O C E V T, E,G,H)L S(T,S,P,VIKS F D R $211 E C=$

$\begin{array}{lllllllllllllll}1 & 2 & 3 & 4 & 5 & 6 & 7 & 8 & 9 & 10 & 11 & 12 & 13 & 14 & 15\end{array}$

1 ASPITHR, SER, SER, SER, GLU,GLU,PRO,MET, ILU)LEU SERISER, GLY, ALA, VAL IASP ARGIASP, THR, THR, SER, SER, GLU,GLU,ALA, VAL, ILU, ILU, ILU,

31 PHE, CYSILEUITYR,ASP, TRP, GLU,GLU,PRO, GLYILYS LYS ALA PRO LYS LEU LEU ILU TYR ASP ALA SER LYS LEU GLUISER, PRO, GLY, ALA, VAL)

61 ARG PHE SER(ASP, THR, THR, SER, GLY,GLY,GLY)PHE THR(ASP, SER, SER, GLU,GLU,PRO,ILU,LEU)ILU ALA THR TYR(ASP,ASP,THR,GLU,GLU,PRO,

91 LEU,LEU, CYS, TYR, PHE, PHEIGLYITHR, GLY,GLYILYS VAL ASP PHE LYS ARg THRISER, PRo, ALA, ALA, VAL IVAL PHE ILUIASP, SER, GLU, GLU, PRo, 121 PRO, PHEILEU LYS SERITHR, SER, GLY, ALAIVALIVAL, CYSILEU LEU ASP IASP,PRO, PHEITYR ARG GLU ALA LYS VAL GLU TRP LYS VALIASP,ASP, 151 ASP, SER, SER, GLU, GLU, GLY, ALA,LEU)GLU SERIASP, THR, SER, GLU, GLU, VALILYS ASPITHR, SERITYR SER SER SER THR LEU LEU THR LEU SER

181 LYS ALA ASP TYR GLU LYS HIS LYS LEU TYR ALA CYS GLU VALITHR, GLU,GLY,HISILEU SERITHR, SER, PRO, VALILYS SER PHE ASP ARG GLY 
BJ HU $10.001-2$

\section{COMPOSITION}

\begin{tabular}{|c|c|c|c|c|c|c|c|c|c|c|}
\hline 13 & ALA & A & 0 & GLN & $Q$ & 17 & LEU & $\mathbf{L}$ & 29 & SER \\
\hline 5 & ARG & $R$ & 24 & GLU & $E$ & 14 & LYS & $k$ & 17 & THR \\
\hline 0 & ASN & $\mathbf{N}$ & 13 & GLY & G & 1 & MET & $M$ & 2 & TRP \\
\hline 20 & ASP & D & 2 & HIS & $H$ & 10 & PHE & $F$ & 8 & TYR \\
\hline 5 & CYS & C & 8 & ILU & I & 11 & PRO & $\mathbf{P}$ & 13 & VAL \\
\hline
\end{tabular}

- hilschmann, N. AND Craig, l.C., proc. natl. acad. SCI. U.S., VOL.53, NO.6, PP.1403-1409, 1965 
ACHER, R。

AMBLER, $R \bullet P$ •

ANDERER , F•A。

ANSFIELD, M. J.

BAHL, $O . P$.

BAGLIONI, C.

BARNAFI, L。

BARTSCH, R• G•

BEALE, D.

BEHRENS, $O \bullet K$.

BELL, P. H.

BENSON, A.

BERNIER, I •

BLAKE, C. C. F.

BLOMBACK, B.
BRAUN I TZER , G.

BROMER, W. W.

BROWN, H.

BROWN, J. R:

BROWN, L. H•

BUETTNER-JANUSCH, J.

CANFIELD, R •

CHAUVET, Jo

CHUNG, D.

COHEN, S•

COLE, D.

CORMICK, J.

CRAIG, L. C.

DAVIS, D. S.

DIXON, J. S.
TI BOPA 5.001

PR BOAR 8.101

CY PS 1.006

AZ PS 3.003

TM TM 6.001

TI BOPA 5.001

CY RS 1.009

GL HUH 2.020

TN BOBM 8.102

TN PGBM 8.203

$\mathrm{DH} \mathrm{CH} \quad 3.001$

GL HUH 2.020

GN BO 8.001

TN PGAC 8.206

CY PG 1.005

FE CP 3.002

LS CH 7.201

LS CH 7.201

FB BOA 9.001

FB SHA 9.002

FB GTA 9.003

FB RDA 9.004

FB PGA 9.005

FB HUA 9.006

FB RTA 9.007

FB BOB 9.101

FB SHB 9.102

FB GTB 9.103

FB RDB 9.104

FE PGB 9.105

FB HUB 9.206

FB RTB 9.107

GL HUHA 2.001

GL HUHB 2.002

GL HOHA 2.006

GN BO 8.001

IS SHA 8.304

IS WPA 8.305

TR BOCH 7.001

AZ PS 3.003

GL LEHB 2.007

LS CH 7.201

TI BOPA 5.001

PR BOAR 8.101

TN SBAC 8.207

10.000

TN SBAC 8.207

GL HUHA 2.001

GL HUHG 2.003

BJ HU 10.001

TN PGAC 8.206

TN BPAM 8.201

TN HOBM 8.204

TN SBAC 8.207

TI BOPA 5.001 
DOOLITTLE, R. F.

DUS, $K$.

DU VIGNEAUD, $V$.

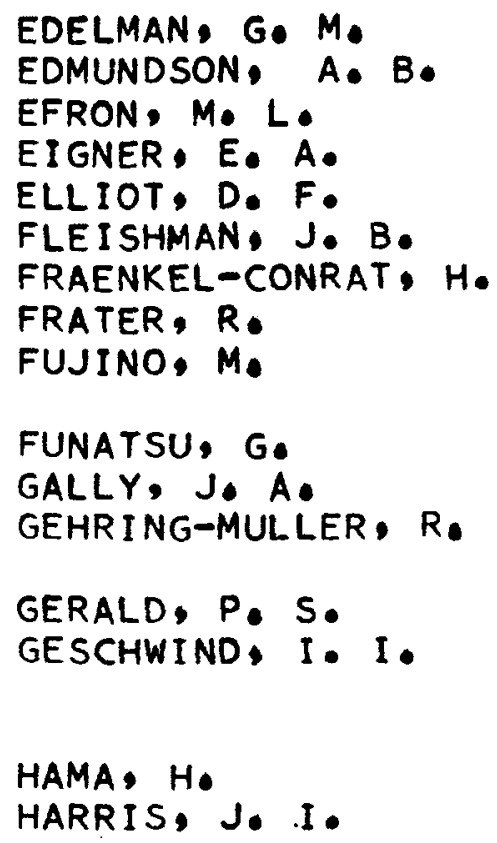

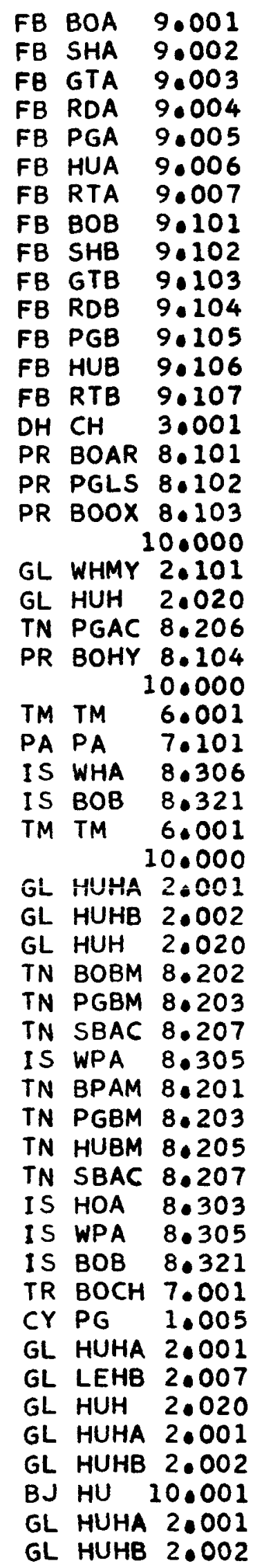




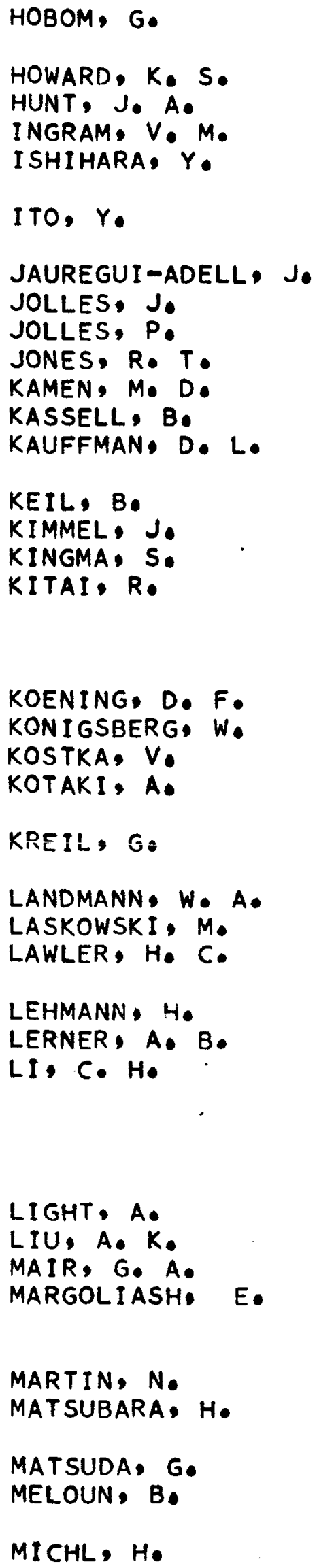

GL HUHA 2.001

GL HUHB 2.002

TN PGAC 8.206

GL HUH 2.020

GL HUH 2.020

IS WHA 8.306

IS BOB B.321

IS WHA 8.306

IS BOB 8.321

LS CH 7.201

LS CH 7.201

LS CH 7.201

GL HUHG 2.003

DH CH 3.001

TI BOPA 5.001

TR BOCH 7.001

TR BOTR 7.002

TR BOCH 7.001

PA PA 7.101

GL HUH 2.020

IS BOA 8.301

IS SHA 8.304

IS WPA 8.305

IS BOB 8.321

LS CH 7.201

GL HUHA 2.001

TR BOCH 7.001

IS BNA 8.302

IS BNB 8.322

CY HO 1.003

CY TF 1.007

TN PGAC 8.206

TI BOPA 5.001

PR BOAR 8.101

PR PGLS 8.102

GL HUH 2.020

TN BPAM 8.201

TN BPAM 8.201

TN BOBM 8.202

TN PGBM 8.203

TN HOBM 8.204

TN SBAC 8.207

PA PA 7.101

LS CH 7.201

LS CH 7.201

CY CH 1.002

CY HO 1.003

CY PG 1.005

GL HUH 2.020

CY HU 1.004

CY PG 1.005

GL HOHA 2.006

TI BOPA 5.001

TR BOCH 7.001

PR BOOX 8.103 


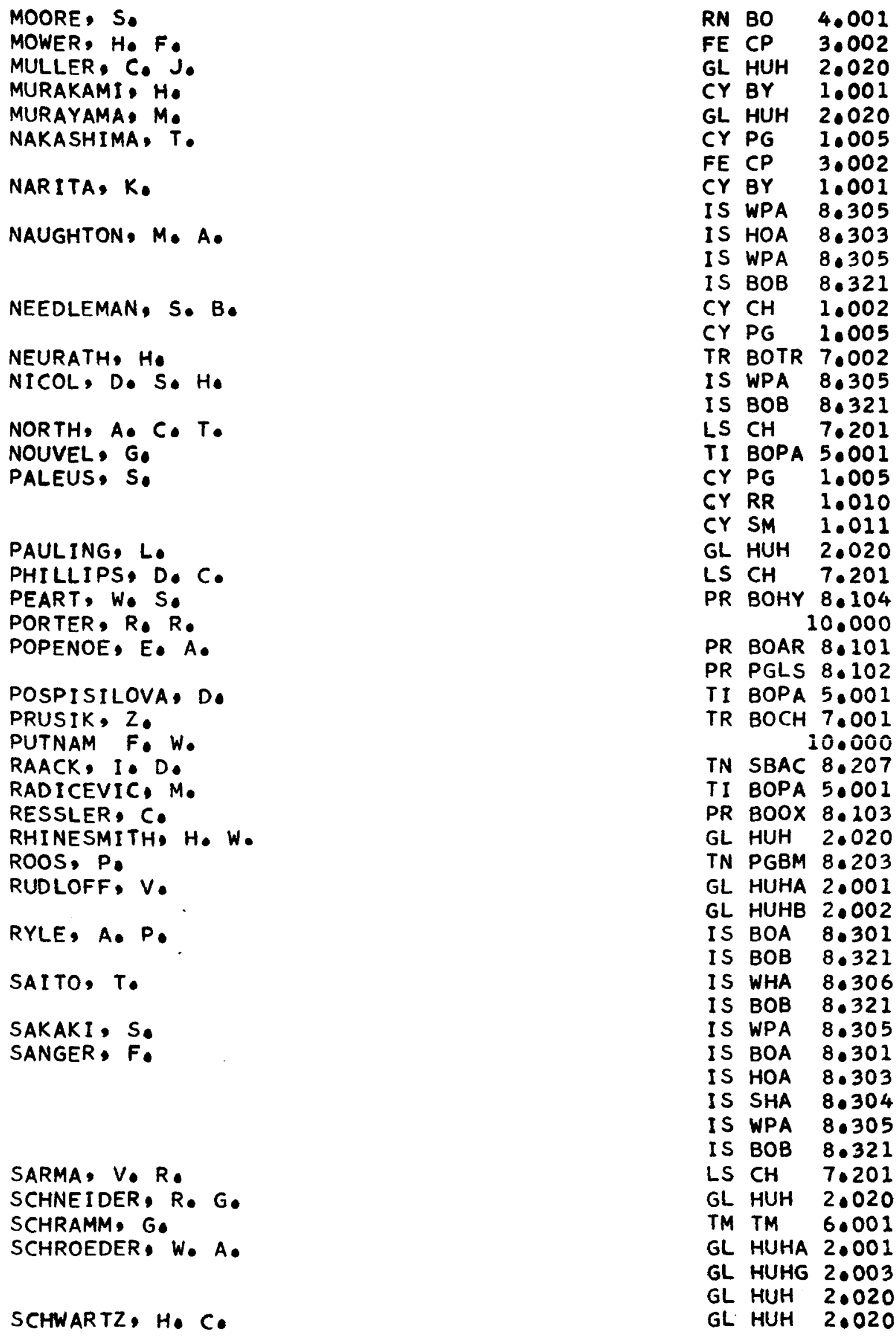

RN BO 4.001

FE CP 3.002

GL HUH 2.020

CY BY 1.001

GL HUH 2.020

CY PG 1.005

FE CP 3.002

CY BY 1.001

IS WPA 8.305

IS HOA 8.303

IS WPA 8.305

IS BOB 8.321

CY CH 1.002

CY PG 1.005

TR BOTR 7.002

IS WPA 8.305

IS $B O B \quad 8.321$

LS CH 7.201

TI BOPA 5.001

CY PG 1.005

CY RR 1.010

CY SM 1.011

GL HUH 2.020

LS CH 7.201

PR BOHY 8.104

10.000

PR BOAR 8.101

PR PGLS 8.102

TI BOPA 5.001

TR BOCH 7.00I 10.000

TN SBAC 8.207

TI BOPA 5.001

PR BOOX 8.103

GL HUH 2.020

TN PGBM 8.203

GL HUHA 2.001

GL HUHB 2.002

IS BOA 8.301

IS BOB 8.321

IS WHA 8.306

IS BOB 8.321

I5 WPA 8.305

IS BOA 8.301

IS HOA 8.303

IS SHA 8.304

IS WPA 8.305

IS $B O B \quad 8.321$

LS CH 7.201

GL HUH 2.020

TM TM 6.001

GL HUHA 2.001

GL HUHG 2.003

GL HUH 2.020

GL HUH 2.020 


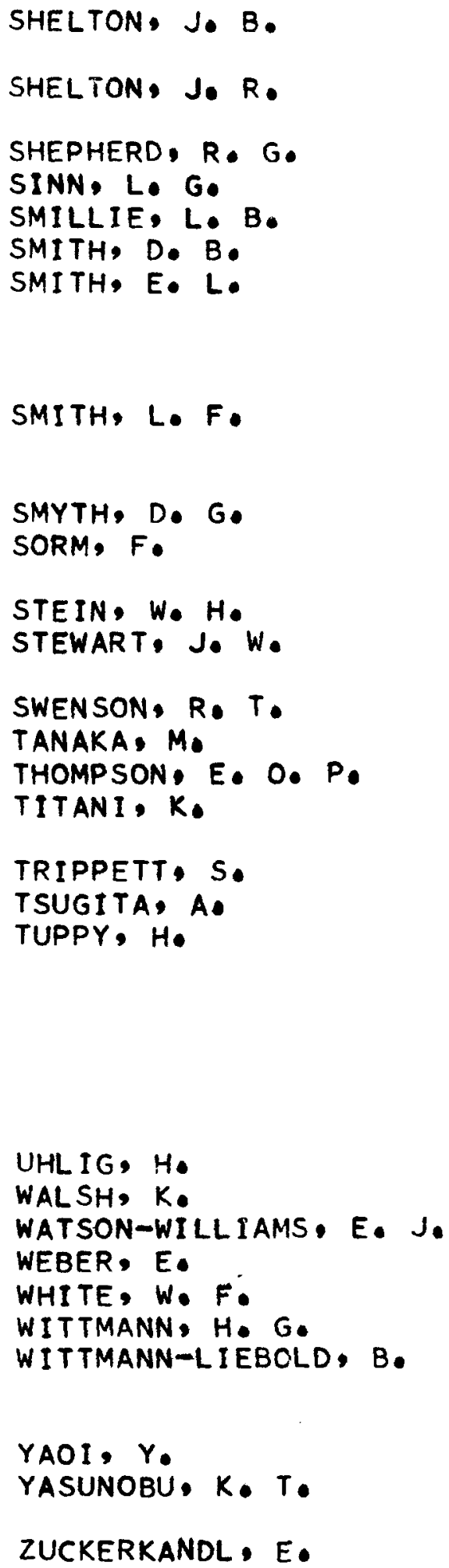

GL HUHA 2.001

GL HUHG 2.003

GL HUHA 2.001

GL HUHG 2.003

TN PGAC 8.206

GN BO 8.001

TR BOCH 7.001

GL HOHB 2.005

CY HO 1.003

CY HU 1.004

CY RS 1.009

PA PA 7.101

IS BOA 8.301

IS WPA 8.305

IS BOB 8.321

RN BO 4.001

TI BOPA 5.001

TR BOCH 7.001

RN BO 4.001

CY CH 1.002

CY PG 1.005

GL HUH 2.020

FE CP 3.002

IS BOA 8.301

CY BY 1.001

IS WPA 8.305

PR BOOX 8.103

TM TM 6.001

CY HO 1.003

CY PG 1.005

CY SW 1.008

CY RR 1.010

CY SM 1.011

PR BOOX 8.103

IS BOB 8.321

TM TM 6.001

TR BOTR 7.002

GL HUH 2.020

TM TM 6.001

TN PGAC 8.206

TM TMD 6.002

GL HUHA 2.001

GL HUHB 2.002

TM TMD 6.002

CY BY 1.001

CY PG 1.005

FE CP 3.002

GL GOHB 2.004 